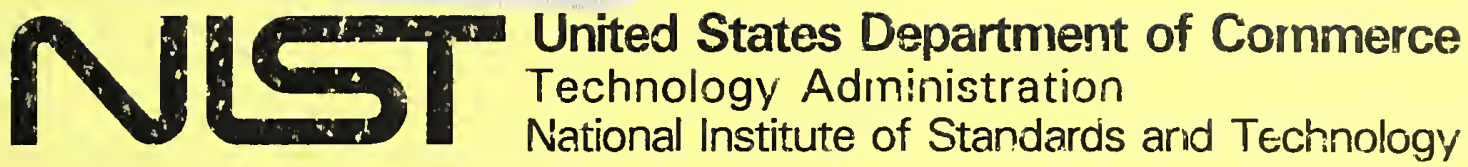

NISTIR 5086

Bibliography of the NIST Optoelectronics Division

Edited by

Annie J. Smith

$Q C$

100

.1156

\$0.5086 



\section{Bibliography of the \\ NIST Optoelectronics Division}

Edited by

Annie J. Smith

Optoelectronics Division

Electronics and Electrical Engineering Laboratory

National Institute of Standards and Technology

Boulder, Colorado 80303-3328

September 1999

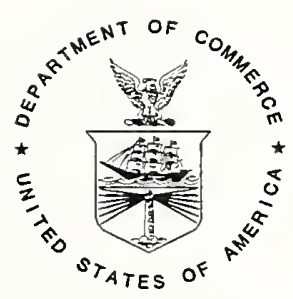

U.S. DEPARTMENT OF COMMERCE, William M. Daley, Secretary 

CONTENTS

Page

INTRODUCTION ....................... 1

SOURCES AND DETECTORS GROUP, 815.01

HIGH SPEED MEASUREMENTS . . . . . . . . . . . . . . . . . 9

LASER RADIOMETRY . . . . . . . . . . . . . . . . 15

FIBER AND INTEGRATED OPTICS GROUP, 815.02

OPTICAL FIBER METROLOGY . . . . . . . . . . . . . . . 25

INTEGRATED OPTIC METROLOGY . . . . . . . . . . . 43

OPTICAL COMPONENTS GROUP, 815.03

OPTICAL FIBER SENSORS . . . . . . . . . . . . . . . . . 46

FIBER AND DISCRETE COMPONENTS . . . . . . . . . . . . . . . . 59

OPTICAL MANUFACTURING GROUP, 815.04

DIELECTRIC MATERIALS AND DEVICES . . . . . . . . . 63

SEMICONDUCTOR MATERIALS AND DEVICES . . . . . . . . . 69

CONFERENCE DIGESTS AND PROCEEDINGS . . . . . . . . . . . 74

MISCELLANEOUS TOPICS . . . . . . . . . . . . . . . . . 77







\title{
A BIBLIOGRAPHY OF THE NIST OPTOELECTRONICS DIVISION
}

\author{
Annie J. Smith, Editor
}

\section{INTRODUCTION}

Gordon W. Day

The National Institute of Standards and Technology's (NIST's) Optoelectronics Division was established in 1994 to provide the optoelectronics industry with measurement technology, standards, and traceability to those standards. Optoelectronics research at NIST is not new, however. The Division's roots extend to the first NIST (then the National Bureau of Standards-NBS) work on lasers-research begun in the early 1960s to develop techniques for measuring their output power or energy.

Laser characterization continues to be an important activity at NIST. The Optoelectronics Division maintains national standards for laser radiometry at a wide range of laser wavelengths, from the ultraviolet through the mid-infrared. The Division and its predecessor organizations have been providing measurement services for laser power and energy since 1967 and each year

Mission
To provide the optoelectronics industry
and its customers and suppliers with
comprehensive and technically advanced
measurement capabilities, standards, and
traceability to those standards.
conduct more than 200 calibrations for about 50 customers. Many of these involve the calibration of an optical detector or power meter that will serve as a local standard at a customer's facility. Customers represent a wide range of applications where an accurate knowledge of laser output is important-materials processing, eye surgery, optical communications, and semiconductor lithography, to name a few.

In the mid-1970s NIST's (NBS's) work in optoelectronics expanded to support the then-infant field of optical communications. At the time, optical fiber manufacturers were spending about $20 \%$ of their production costs on measurements, and they often could not agree with their customers on the characteristics of the fiber they sold. NIST has worked on these problems with manufacturers and users, primarily through industry standards organizations. Today, measurement quality in the industry is much better and costs are much lower. High quality instrumentation for characterizing optical fiber is available commercially, and much of the Division's work has shifted to the development of artifact standards to calibrate such instrumentation. As other, newer products pass through this cycle, the Division continues to assist, providing measurement expertise and often serving as a neutral party, in the development of voluntary industry standards. 
High measurement costs are not acceptable in manufacturing, but better measurements can sometimes serve as a means of reducing manufacturing costs. Measurements made during manufacturing can be used to control the process more effectively, allowing more automation and leading to less waste and higher quality products. Recognizing that these issues are particularly important in complex optoelectronic devices, the Division devotes a significant portion of its resources to the development of measurements for efficient manufacturing.

The Division maintains close connections to the optoelectronics industry. Division staff members represent NIST and the U.S. to the major domestic and international standards organizations active in optoelectronics-the Telecommunications Industry Association (TIA), the International Electrotechnical Commission (IEC), the International Organization for Standardization (ISO), and the American National Standards Institute (ANSI)-and provide impartial technical expertise in their negotiations. The Division typically has several active Cooperative Research and Development Agreements (CRADAs) with companies and universities, and is involved in 20 to 30 additional informal collaborations each year. The Division offers the optoelectronics community an annual short course on laser measurements and biennially organizes a major international conference on optoelectronic measurements, the Symposium on Optical Fiber Measurements.

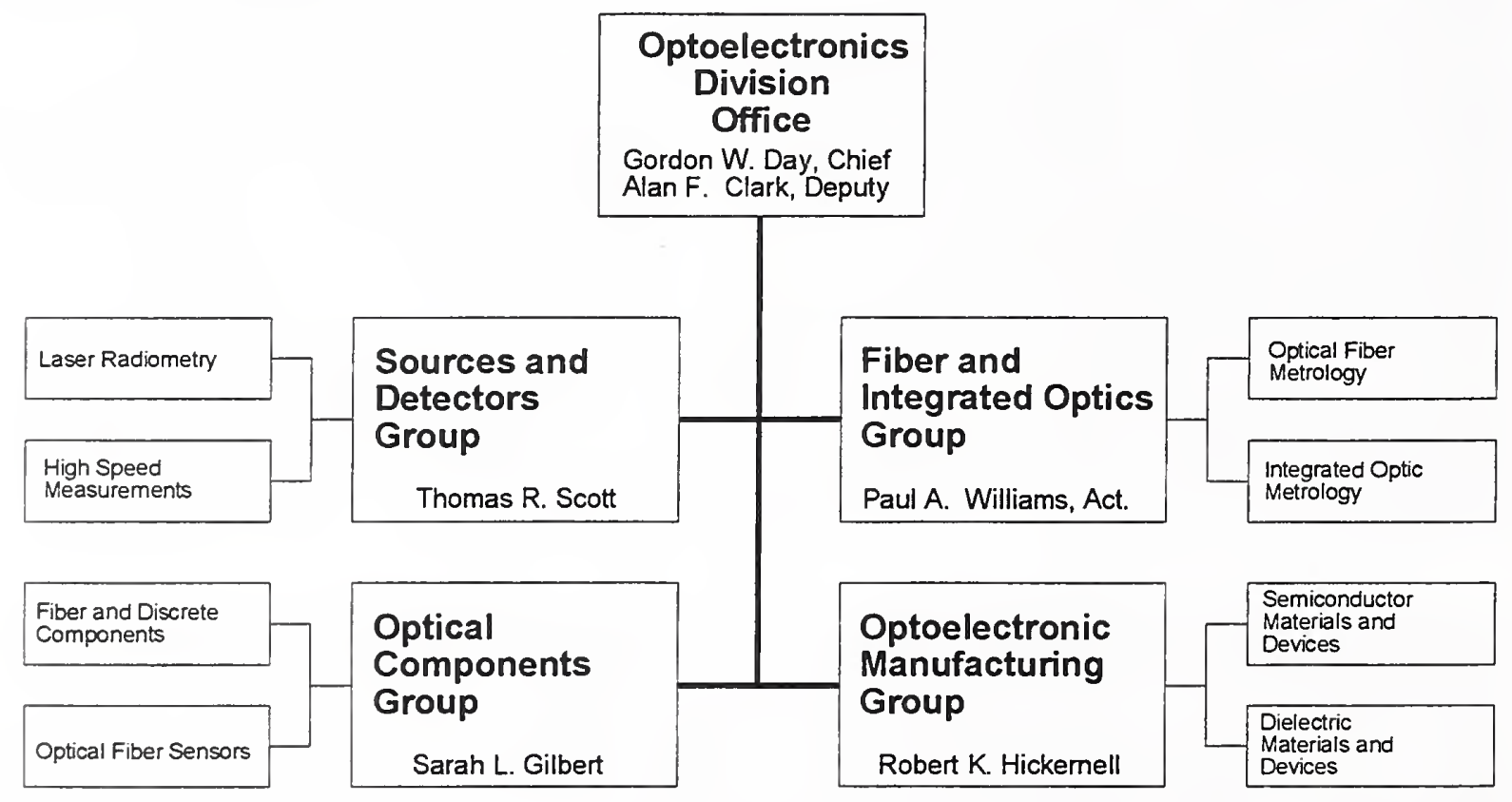

The Division is organized into four groups: the Sources and Detectors Group, the Fiber and Integrated Optics Group, the Optical Components Group, and the Optoelectronic Manufacturing Group, which work in eight project areas, as shown above and described below. 


\section{Laser Radiometry}

The Laser Radiometry Project develops measurement methods and standards for characterizing laser sources and detectors used primarily with $\mathrm{cw}$ or pulsed radiation, and develops and maintains measurement services for laser power and energy, optical fiber power, and related parameters.

Accurate characterization of optoelectronic sources and detectors is important in the development and use of industrial technologies such as lightwave telecommunications, laser-based medical instrumentation, materials processing, photolithography, data storage, and laser safety equipment. This Project focuses on selected critical parameters intrinsic to optoelectronic sources and detectors, especially the calibration of optical fiber power meters and laser power or energy meters at commonly used wavelengths and powers or energies. In addition, special test measurements are available for linearity, spectral responsivity, and spatial uniformity of laser power meters and detectors. In support of source characterization, measurement methods are developed to evaluate and reduce laser intensity noise, and to characterize beam intensity profile and propagation of laser beams. The semiconductor photolithography and corneal sculpting markets require pulsed excimer laser measurements. Project members participate in national and international standards committees developing standards for laser safety, laser radiation measurements (such as beam profile and pointing stability), and optical-power-related measurements. They extend and improve source and detector characterizations, including development of low noise, spectrally flat, highly uniform pyroelectric detectors, high accuracy transfer standards for optical fiber and laser power measurements, and advanced tunable laser systems for laser power and energy measurement systems.

\section{High Speed Measurements}

High-bandwidth measurements are needed to support high-performance systems which take advantage of the potential bandwidth of optical fiber. Systems presently being installed operate at 5 to 10 gigabits per second (a bit rate of gigahertz) using pure optical time division multiplexing (OTDM). Research is being done on the next generation of OTDM systems at bit rates of 20 to $40 \mathrm{GHz}$ in laboratories around the world. Methods are needed to characterize the frequency and impulse response of high speed sources and detectors to at least the third harmonic of the system modulation rate. Burst mode operation in asynchronous transfer mode networks requires characterization at very low frequencies. Increasingly tight tolerances in both digital and analog systems require frequency response measurements with low uncertainty. NIST has developed highly accurate heterodyne techniques at $1319 \mathrm{~nm}$ and $850 \mathrm{~nm}$ for measuring detector frequency response. High-speed optoelectronic system design requires measurement of the phase response of optoelectronic components. Researchers in the High-Speed Measurements Project are developing time-domain techniques for measuring optoelectronic phase response with verifiable accuracy. 
Source and detector noise measurements are required to predict low bit error ratios in computer interconnects, high carrier-to-noise ratios in analog systems, and to support erbium-doped fiber amplifier noise figure measurements. NIST is developing methods for calibrating optical noise measurement systems. The intensive use of laser target designators by the armed forces requires traceable low level pulse power and energy calibration standards at $1.06 \mu \mathrm{m}$ and $1.55 \mu \mathrm{m}$. NIST has developed methods for calibrating absolute laser pulse energy and peak power which are traceable to national CW laser power and energy standards maintained by the Sources and Detectors Group.

\section{Optical Fiber Metrology}

The Optical Fiber Metrology project develops measurement methods and Standard Reference Materials for optical fibers, and interacts with standards groups to provide a metrology base for the lightwave communication industry.

Optical fibers have largely replaced coaxial cable in long-distance telecommunications systems and are rapidly being installed in local-area applications. NIST staff interact with industry groups to develop measurement methods and reference standards for the characterization of these components. Connecting optical fibers together requires the fibers to have accurately controlled dimensions. NIST-developed Standard Reference Materials (SRM) for cladding diameter allow manufacturers to calibrate instrumentation used in manufacturing and quality control; geometrical standards for fiber coatings, connector ferrules, and mode-field diameter are also available as SRMs. Dispersion, the variation of propagation velocity with wavelength or polarization, sets the limit to the rate at which information can be transmitted; measurement methods and standards for chromatic dispersion are available and polarization-mode dispersion standards are currently under development. The development of optical amplifiers has brought revolutionary changes to the design of communications; these new components require special methods of characterization now in development. Nonlinear properties of fiber such as four-wave mixing and solitons have been studied and applied to instrumentation. Novel implementations of reflectometry in fiber are also studied. New methods of characterizing multimode fiber bandwidth with application to high speed, gigabit local area data networks have also been developed.

\section{Integrated Optic Metrology}

Optical waveguides in planar geometries, often called integrated optics, are increasingly used in communications and other optoelectronic systems. This Project develops measurement methods for integrated optic waveguides. As more optical fiber is used in local area networks and moves closer to the home and desk, more passive components, such as splitters and couplers will be used. The trend to wavelength division multiplexing also adds to the demand for such components. Devices with large numbers of ports are likely to be less costly to produce using planar waveguide technology rather than fiber technology. Several companies now manufacture 1 $\mathrm{x} \mathrm{N}$ splitters using planar technology or are about to market them. There are, however, no standard measurement procedures or artifact standards similar to those for optical fiber. Nor is it 
obvious how to perform analogous measurements when the mode field pattern of an integrated optical waveguide is not circularly symmetric or when the normal fiber measurement is performed using a cutback technique. The Integrated Optic Metrology Project is using such tools as low coherence reflectometry and refracted near-field scanning to address those measurement issues which are peculiar to planar waveguide geometries.

\section{Optical Fiber Sensors}

The NIST Optical Fiber Sensors project supports the optoelectronics industry by providing measurements and standards for the characterization of optical materials and components used in fiber optic sensors, communications systems, and polarimetric instruments. We also develop and evaluate new fiber optic sensor systems, components, and transducers for other government agencies and industry. When possible, newly developed sensor technology is transferred to interested U.S. companies; one recent success is a commercially available current sensor made from common optical fiber that uses a process developed at NIST.

The polarimetry and interferometry expertise developed from sensor work is also applied to general component measurements. We are using low-coherence interferometry to characterize dispersion and other properties of fiber Bragg gratings. The Project has developed a Standard Reference Material for optical retardance and is working with industry groups to improve retardance measurements in optical discs and photolithography masks. We are developing capabilities in imaging Mueller -matrix polarimetry and offer special measurement services for retardance using the accurate methods developed at NIST.

\section{Fiber and Discrete Components}

Advanced optical communications systems use many different types of components to control and modify propagating signals. The Fiber and Discrete Components Project develops measurement technology to characterize these components and understand their limits.

This project is currently conducting research on polarization-dependent loss metrology, optical fiber photosensitivity, and wavelength standards for optical fiber communications. Polarization-dependent loss in components affects a system's performance, especially when many components are in the system. We are developing a polarization-dependent loss calibration standard for commercial test instruments. Photo-induced Bragg gratings in optical fiber are important components of wavelength filters and dispersion compensators for wavelength division multiplexed (WMD) optical fiber communications systems, and are also excellent strain sensors. The project conducts research on the photosensitivity mechanism responsible for the grating inscription process in optical fiber and other materials. . The project is also developing Standard Reference Material absorption cells for wavelength calibration in the WDM region. These cells can be used to calibrate the instruments that characterize the spectrum of sources and the wavelength dependence of components. This calibration capability will become increasingly important as more WDM optical communication systems are implemented. 


\section{Dielectric Materials and Devices}

NIST is developing measurement methods and acquiring critical materials data to improve the manufacturing of dielectric optical devices. Of current concern are the ferroelectric materials, lithium niobate and lithium tantalate, used in optical waveguide modulators, switches, and micro-lasers, and the glasses used in waveguide splitters, couplers, lasers, and optical amplifiers. Using nonlinear optical analysis, NIST researchers are measuring the compositional uniformity of substrate material and deposited layers. Rapid, nondestructive testing of uniformity across a wafer, or from wafer to wafer, aids substrate and device manufacturers in processing decisions which increase device yield. Additionally, various periodically poled structures for specialized frequency conversion applications, optical parametric oscillators (OPOs), etc., are under investigation. In collaboration with industry and with support from other governmental agencies, NIST is improving methods for the manufacture of compact, rare-earth-doped waveguide lasers and amplifiers. This work includes ultrafast passively mode-locked and Q-switched waveguide lasers.

Measurements of dopant diffusion and solubility in substrate material and the absorption and emission spectra of doped waveguides are combined with computer models to characterize the influence of glass chemistry, ferroelectric domain-reversed regions, and other critical parameters. Grating-based integrated optical components are gaining importance for wavelength division multiplexing, dispersion compensation, single-frequency lasers, and optical sensors. NIST scientists in the Dielectric Materials and Devices Project are studying the processing and metrology issues which lead to compact, robust $\mathrm{cw}$ and pulsed solid-state laser and nonlinear optical devices having high efficiency.

\section{Semiconductor Materials and Devices}

The commercial success of semiconductor optoelectronic devices requires low-cost manufacturing for an ever-widening array of applications: telecommunications, computer interconnects, data storage, display, printing, and sensor systems. NIST scientists in this project develop measurement methods and provide data to support the efficient manufacture of devices such as diode lasers, light-emitting diodes, photodetectors, and modulators. They also invent and develop advanced devices to support research in NIST, other government agencies, and industry.

This Project is investigating the potential and limitations of optical instrumentation for the in situ monitoring of compound semiconductor epilayer deposition. Researchers correlate data from several in situ and ex situ measurement techniques to improve the measurement accuracy, with the goal of developing standard test procedures and reference materials. The project has begun to address the issue of impurity evaluation in source gases used for epitaxial deposition. Collaborative research has led to the high-resolution probing of vertical-cavity, surface-emitting laser (VCSEL) emission by near-field scanning optical microscopy, and to the measurement of the dielectric constants of native oxides used in VCSEL fabrication. Ultrafast pulse measurement capability is applied to materials characterization and next-generation, high-speed communications research. Researchers in this project fabricate semiconductor quantum well and quantum dot 
devices (in-plane lasers, VCSELs, high-speed resonant-cavity photodiodes, and saturable absorbers, for example) for use in metrology, sensing, and the understanding of manufacturing process limitations. The Optoelectronic Manufacturing Group combines its expertise to demonstrate hybrid integrated devices such as pulsed, solid-state waveguide lasers.

\section{Publications}

This bibliography provides one means of access to the work of the division. It includes most of the papers published by the Division and its predecessor organization since 1970. A few important earlier papers and a few papers published by present NIST staff before they joined the Institute are included. The document is organized by subject, along the project lines defined above. It is updated annually. The references are presented in inverse chronological order with the most recent first and followed by an author index

\section{Purchase Procedures}

NIST (NBS) Technical Notes, Special Publications, Handbooks, Journals of Research, and Monographs may be purchased from the U.S. Government Printing Office at the following address: New Orders, Superintendent of Documents, P.O. Box 371954, Pittsburgh, PA 152507954, (202) 512-1800. Orders may be paid by major credit card, NTIS Deposit Account, or check or money order payable in U.S. dollars to the Superintendent of Documents. The Government Printing Office usually stocks these publications for only a year or two, after which they may be purchased from the National Technical Information Service at the address listed below. The GPO web site is http://www.access.gpo.gov/.

NIST (NBS) Interagency/Internal Reports (NISTIRs, NBSIRs) may be purchased from the National Technical Information Service, Springfield, VA 22161, (703) 487-4650. Orders may be paid by major credit card, NTIS Deposit Account, or check or money order payable in U.S. dollars to NTIS. The NTIS web site for information and ordering is http://www.ntis.gov/.

Reprints of papers published in non-NIST media may be available in limited quantities from the authors.

\section{A Note on Abbreviations}

On August 23, 1988, the National Bureau of Standards (NBS) became the National Institute of Standards and Technology (NIST); therefore, documents with either prefix are considered NIST publications. 
Most readers are familiar with the commonly used abbreviations for the names of the professional journals that appear in this bibliography. Some publication series are peculiar to NIST and may call for explanation. They are:

NISTIR - NIST Interagency/Internal Report

NIST TN - NIST Technical Note

NIST SP - NIST Special Publication

NIST HB - NIST Handbook

NIST JRES - Journal of Research of the NIST

NIST MN - NIST Monograph
NBSIR - NIST Interagency/Internal Report

NBS TN - NIST Technical Note

NBS SP - NIST Special Publication

NBS HB - NIST Handbook

NBS JRES - Journal of Research of the NBS

NBS MN - NIST Monograph

\section{Acknowledgments}

A large part of the labor of preparing a bibliography is spent collecting and arranging the material; Sheila Aaker, Frances Brown, Linda S. Derr, Edie DeWeese, Kit Kline, and Jessie Page assisted with these chores. The main sources of material were the NIST Boulder Editorial Review Board database, the National Technical Information Service, and information supplied by individual authors. 


\section{HIGH SPEED MEASUREMENTS}

How to Measure Relative Intensity Noise in Lasers;

Obarski, G.E.; Hale, P.D.

Laser Focus World 35(5): 273-277; May 99

Unraveling the Mysteries of Intense Femtosecond Pulse Propagation;

Diddams, S.A.; Eaton, H.E.; Van Engen, A.G.; Zozulya, A.A.; Clement, T.S. Opt. and Phot. News 9(12): 37-38; Dec 98

Investigations of Nonlinear Femtosecond Pulse Propagation with the Inclusion of Raman, Shock and Third Order Phase Effects;

Zozulya, A.A.; Diddams, S.A.; Clement, T.S.

Phys. Rev. A 58(4): 3303-3310; Oct 98

Calibration Service for Laser Power and Energy at $248 \mathrm{~nm}$;

Leonhardt, R.W.

NIST TN 1394, 34 pp; Jan 98

Polarization States Behave as Hyperfine Levels in VCSEL Mode Partition Noise;

Obarski, G.E.; Jones, R.D.

OSA TOPS on Advances in Vertical-Cavity Surface Emitting Lasers, 1997 15: 62-68; 97

Relative Intensity Noise Correlates with Beam Profile in an $\mathrm{LP}_{11}$ Mode Vertical-Cavity Surface Emitting Laser;

Obarski, G.E.; Jones, R.D.

Tech. Dig., Conf. on Lasers and Electro-Optics (CLEO '97), Vol 11, May 1823, 1997, Baltimore, Maryland, 228-229; May 97

Relative Intensity Noise of an InGaAsP Laser Over a $22 \mathrm{GHz}$ Bandwidth at Cryogenic and Room Temperature;

Obarski, G.E.; Larson, D.R.; Phelan, R.J., Jr.

Tech. Dig., OSA Topical Mtg., Integrated Photonics Research, Apr 29-May 2, 1996, Boston, MA, Vol 6, 224-227; 96

Frequency Response Measurement of Digital Communications Analyzer Plug-In Modules;

Park, R.; Hale, P.D.

Hewlett-Packard J. 47(6): 37-40; Dec 96

A Transfer Standard for Measuring Photoreceiver Frequency Response;

Hale, P.D.; Wang, C.-M.; Park, R.; Lau, W.Y.

J. Lightwave Tech. 14(11): 2457-2466; Nov 96 
High Speed Measurements

Low-Level Pulsed 1064 nm Laser Radiometer Transfer Standard;

Leonhardt, R.W.

Proc., SPIE, Vol 2815, The International Society for Optical Engineering, Optical Radiation Measurements III, Aug 4-9, 1996, Denver, CO: 154-159; Aug 96

Multi-Gigahertz Relative Intensity Noise of an InGaAsP Laser at Cryogenic Temperature;

Obarski, G.E.; Larson, D.R.; Phelan, R.J., Jr.

Proc., SPIE, Vol 2684, High-Speed Semiconductor Laser Sources, Feb 1-2, 1996, San Jose, CA: 84-91; Feb 96

Deep-UV Excimer Laser Measurements at NIST;

Leonhardt, R.W.; Scott, T.R.

Proc., SPIE, Vol 2439, Microlithography '95, Feb 19-24, 1995, Santa Clara, CA: 448-459; Apr 1, 95

Comparison of Photodiode Frequency Response Measurements to $40 \mathrm{GHz}$ between NPL and NIST;

Gifford, A.D.; Humphreys, D.A.; Hale, P.D.

Electron. Lett. 31(5): 397-398; Mar 2, 95

Photodetector Frequency Response Measurements at NIST US and NPL UK: Preliminary Results of a Standards Laboratory Comparison;

Hale, P.D.; Humphreys, D.A.; Gifford, A.D.

Proc., SPIE, Vol 2149, Technologies for Optical Fiber Communications, Jan 24-28, 1994, Los Angeles, CA: 345-356; Jan 94

Accurate Characterization of High Speed Photodetectors;

Hale, P.D.; Franzen, D.L.

Proc., SPIE, Vol 2022, Tech. Conf., Photodetectors and Power Meters, Jul 15-16, 1993, San Diego, CA: 218-227; 93

High-Speed Lightwave Measurements;

Franzen, D.L.

Dig., XXIVth General Assembly of the Intl. Union of Radio Science, Kyoto, Japan; Aug 93

High-Frequency Optical FM Noise Reduction Employing a Fiber-Insertable Feedforward Compensation Technique;

Esman, R.D.; Iwashita, I.

Tech. Dig., Optical Fiber Communication Conf. (OFC '92), 72; Feb 92 
Errors in the Measurement of Non-Gaussian Noise Spectra Using rf Spectrum Analyzers; Anderson, M.H.; Jones, R.D.; Cooper, J.; Smith, S.J. Rev. Sci. Instrum. 62(11): 2828-2830; Nov 91

Lambdameter for Accurate Stability Measurements of Optical Transmitters; Obarski, G.E. Proc., SPIE, Vol 1620: 41-48; Nov 91

Improved Low-Level Silicon Avalanche Photodiode Transfer Standards at 1064 Micrometers; Rasmussen, A.L.; Sanders, A.A.; Simpson, P.A. NISTIR 89-3917, 39 pp; Aug 22, 89

An Electrically Calibrated Silicon Bolometer for Low Level Optical Power and Energy Measurements;

Phelan, R.J., Jr.; Craig, R.M.

Proc., SPIE, Vol 888: 38-42; 88

Fast-Pulse Generators and Detectors for Characterizing Laser Receivers at $1.06 \mu \mathrm{m}$;

Simpson, P.A.

Proc., SPIE, Vol 888: 43-47; 88

Fast Optical Detector Deposited on Dielectric Channel Waveguides;

Larson, D.R.; Phelan, R.J., Jr.

Opt. Eng. 27(6): 503-506; Jun 88

Picosecond Pulse Response from Hydrogenated Amorphous Silicon (a-Si:H) Optical Detectors on Channel Waveguides;

Larson, D.R.: Phelan, R.J., Jr.

Proc., SPIE, Vol 835: 59-63; 87

Direct Measurement of the Spatial Modes of a Laser Pulse-Theory;

Johnson. E.G.. Jr.

Appl. Opt. 25(17): 2967-2975; Sep 86

Transfer Standards for Energy and Power of Low-Level $1.064 \mu \mathrm{m}$ Laser Pulses and cw;

Rasmussen. A.L.; Sanders, A.A.

Opt. Eng. 25(2): 277-285; Feb 86

A Sensitive High Frequency Electromagnetic Field Probe Using a Semiconductor Laser in a Small Loop Antenna;

Phelan, R.J., Jr.; Larson, D.R.; Simpson, P.

Proc., SPIE, Vol 566: 300-306; 85 
High Speed Measurements

A Calorimeter for Measuring 1-15 kJ Laser Pulses;

Simpson, P.A.; Johnson, E.G., Jr.

Proc., SPIE, Vol 499: 121-124; 85

Documentation of the NBS APD and PIN Calibration Systems for Measuring Peak Power and Energy of Low-Level $1.064 \mu \mathrm{m}$ Laser Pulses;

Rasmussen, A.; Sanders, A.A.

NBSIR 85-3032, 67 pp; Dec 85

Low-Level Germanium Detector Transfer Standard at $1.064 \mu \mathrm{m}$;

Rasmussen, A.; Sanders, A.A.

NBSIR 85-3041, 10 pp; Dec 85

Direct Measurement of the Electric Field of a Laser Pulse-Theory;

Johnson, E.G., Jr.

NBS TN 1084, 52 pp; Aug 85

Fast Detectors and Modulators;

Phelan, R.J., Jr.

Chap. in Semiconductors and Semimetals, Academic Press, 21(D): 249-259; 84

A Calorimeter for Measuring High-Energy Optical Pulses;

Simpson, P.A.; Etzel, S.M.; Johnson, E.G., Jr.

NBSIR 84-3008, 162 pp; Oct 84

A Calorimeter for Measuring 1-15 kJ Laser Pulses;

Simpson, P.A.; Johnson, E.G., Jr.

Proc., SPIE, Vol 499: 34-37; Aug 84

Detectors for Picosecond Optical Power Measurements;

Phelan, R.J., Jr.; Larson, D.R.; Frederick, N.V.; Franzen, D.L.

Proc., SPIE, Vol 499: 34-37; Aug 84

The Use of Power Transfer Matrices in Predicting System Loss: Theory and Experiment; Maisonneuve, J.M.; Gallawa, R.L.

Proc., SPIE, Vol 500: 88-93, and Fiber Integ. Opt. 6(1): 11-26; Aug 84

Using Optical Processing to Find the Beam Profile of a Laser Pulse-Theory;

Johnson, E.G., Jr.

Proc., SPIE, Vol 499: 75-88; Aug 84 
A Computer Controlled System for Calibrating Detectors of TEA Laser Pulses;

Simpson, P.A.

Proc., 22nd Automatic rf Testing Group Conf., Nov 3-4, 1983, Albuquerque, NM, ARFTG Fall Dig., 25-36; 83

Submicrometer Interdigital Silicon Detectors for the Measurement of Picosecond Optical Pulses;

Phelan, R.J., Jr.; Larson, D.L.; Frederick, N.V.; Franzen, D.L.

Proc., SPIE, Vol 439: 207-211; 83

A System for Measuring Energy and Peak Power of Low-Level 1.064 $\mu$ m Laser Pulses;

Sanders, A.A.; Rasmussen, A.L.

NBS TN 1058; Oct 82

Sub-Nanosecond Electrical Modulation of Light with Hydrogenated Amorphous Silicon;

Phelan, R.J., Jr.; Larson, D.R.; Werner, P.E.

Appl. Phys. Lett. 38(8); Apr 15, 81

A System for Characterizing Detectors for the Measurement of Power of $\mathrm{CO}_{2}$ TEA Laser Pulses;

Simpson, P.A.

Proc., 11th Annual Electro-Optical Laser Conf., Oct 23-25, 1979, 3999-4007; 80

A System for Measuring the Characteristics of High Peak Power Detectors of Pulsed $\mathrm{CO}_{2}$ Laser Radiation;

Simpson, P.A.

NBS TN 1023; Sep 80

Measuring Features of the Fluence at the Far Field of a $\mathrm{CO}_{2}$ Pulsed Laser-An Issue Study With Suggestions on How To Do It;

Johnson, E.G., Jr.

NBSIR 80-1628; Apr 80

LED Source for Determining Optical Detector Time Response at 1.06 Micrometers;

Franzen, D.L.; Day, G.W.

Rev. Sci. Instrum. 50: 1029; 79

Measurement of Low Level Laser Pulses at $1.064 \mu \mathrm{m}$;

Rasmussen, A.L.; Sanders, A.A.

Proc., SPIE, Vol 196: 96-103; 79 
High Speed Measurements

Proposed Standards for Laser Signatures;

Danielson, B.L.

NBSIR 77-856; Apr 77

Absolute Reference Calorimeter for Measuring High Power Laser Pulses;

Franzen, D.L.; Schmidt, L.B.

Appl. Opt. 15: 3115-3122; Dec 76

Ultraviolet Laser Action from $\mathrm{Cu}$ II in the 2500-A Region;

McNeil, J.R.; Collins, G.J.; Persson, K.B.; Franzen, D.L.

Appl. Phys. Lett. 28: 207-209; Feb 76

Speed of Light from Direct Frequency and Wavelength Measurements of the

Methane-Stabilized Laser;

Evenson, K.M.; Wells, J.S.; Petersen, F.R.; Danielson, B.L.; Day, G.W.

Phys. Rev. Lett. 29: 1346-1349; Nov 72

Gain Saturation Measurements in $\mathrm{CO}_{2}$ TEA Amplifiers;

Franzen, D.L.; Jennings, D.A.

J. Appl. Phys. 43: 729-730; Feb 72 


\section{LASER RADIOMETRY}

A Transfer Standard for Optical Fiber Power Metrology;

Lehman, J.; Li, X.

Opt. and Phot. News, Eng. and Lab. Notes, 10(5), 44f-h; May 99

Thermal Characterization of a Cryogenic Radiometer and Comparison with a Laser Calorimeter;

Livigni, D.J.; Cromer, C.L.; Scott, T.R.; Johnson, B.C.; Zhang, Z.M. Metrología 35: 819-827; 98

Deep Ultraviolet Laser Metrology for Semiconductor Photolithography;

Dowell, M.L.; Cromer, C.L.; Leonhardt, R.W.; Scott, T.R.

Proc., 1998 Intl. Conf. on Characterization and Metrology for ULSI Tech., May 23-27, 1998, Gaithersburg, MD, Vol 449, 530-541; Nov 98

Heat Transfer Analysis and Modeling of a Cryogenic Laser Radiometer;

Johnson, B.C.; Kumar, A.R; Zhang, Z.M.; Livigni, D.J.; Cromer, C.L.; Scott, T.R.

J. Thermosphys. and Heat Transfer 12(4): 575-581; Oct-Dec 98

Silicon Wedge-Trap Detector for Optical Fibre Power Measurements;

Lehman, J.; Sauvageau, J.; Vayshenker, I.; Cromer, C.L.; Foley, K.

Meas. Sci. Technol. 9(10): 1694-1698; Oct 98

Bicell Pyroelectric Optical Detector Made from a Single $\mathrm{LiNbO}_{3}$ Domain-Reversed Electret;

Lehman, J.H.; Aust, J. A.

Appl. Opt. 37(19): 4210-4212; Jul 1, 98

Pyroelectric Trap Detector for Spectral Responsivity Measurements;

Lehman, J.

Opt. Photon. News 8(11); 35-36; Appl. Opt. 36(34), 97; Nov 97

Optical Radiation Detectors for Laser Measurements: Survey and Tutorial;

Lehman, J.H.; Scott, T.R.

Proc., Intl. Laser Safety Conf., March 17-20, 1997, Orlando, FL, 279-288;

Jul 97

FO Connector Types Affect Power Measurements;

Vayshenker, I.; Li, X.; Keenan, D.A.; Scott, T.R.

Test Meas. World: 23 \& 25; Feb 97 
Laser Radiometry

Errors Due to Connectors in Optical Fiber Power Meters;

Vayshenker, I.; Li, X.; Keenan, D.A.; Scott, T.R.

Tech. Dig., Symp. on Optical Fiber Meas., in NIST SP 905, 49-52; Oct 96

Nonlinearity of Optical Fiber Power Meters;

Vayshenker, I.; Yang, S.; Li, X.; Scott, T.R.

Tech. Dig., Symp. on Optical Fiber Meas., in NIST SP 905, 101-104; Oct 96

Thermal Modeling and Analysis of Laser Calorimeters;

Zhang, Z.M., Livigni, D.J.; Jones, R.D.; Scott, T.R.

J. Thermophys. Heat Transfer 10(2): 350-356; Apr-Jun 96

Automated Measurements of Nonlinearity of Optical Fiber Power Meters;

Vayshenker, I.; Yang, S.; Li, X.; Scott, T.R.

Proc., SPIE, Vol 2550, Photodetectors and Power Meters II, Jul 9-14, 1995 ,

San Diego, CA: 12-19; 95

Characterization of a Clipped Gaussian Beam;

Jones, R.D.; Scott, T.R.

Proc., SPIE, Vol 2375, Photonics West ' 95 Conf., Feb 6-7, 1995, San Jose,

CA: $360-374 ; 95$

Thermal Modeling and Analysis of Laser Calorimeters;

Zhang, Z.M.; Livigni, D.J.; Jones, R.D.; Scott, T.R.

Proc., 1995 ASME/AIAA Natl. Heat Transfer Conf., Aug 6-9, 1995, Portland, OR; Aug 1, 95

Optical Detector Nonlinearity: Simulation;

Yang, S.; Vayshenker, I.; Li, X; Zander, M.; Scott, T.R.

NIST TN 1376, 36 pp; May 95

Accurate Measurement of Optical Detector Nonlinearity;

Yang, S.; Vayshenker, I.; Li, X.; Scott, T.R.

Proc., Natl. Conf. Stds. Labs., Jul 31-Aug 4, 1994, Chicago, IL, 353-362;

Jul 94

Optical Detector Nonlinearity: A Comparison of Five Methods;

Yang, S.; Vayshenker, I.; Li, X.; Scott, T.R.

Tech. Dig., Conf. on Prec. Electromagn. Meas., Jun 27-Jul 1, 1994, Boulder, CO, 455-456; Jul 94 
Optical Power Meter Calibration Using Tunable Laser Diodes;

Vayshenker, I.; Li, X.; Scott, T.R.

Proc., Natl. Conf. Stds. Labs., Jul 31-Aug 4, 1994, Chicago, IL, 363-372;

Jul 94

Spatial Uniformity of Optical Detector Responsivity;

Livigni, D.J.; Li, X.

Proc., Natl. Conf. Stds. Labs., Jul 31-Aug 4, 1994, Chicago, IL, 337-352;

Jul 94

Widths and Propagation of a Truncated Gaussian Beam;

Jones, R.D.; Scott, T.R.

Proc., 2nd Workshop on Laser Beam Characterization, May 30-Jun 1, 1994,

Berlin, Germany, 161-172; Jun 94

Error Propagation in Laser Beam Spatial Parameters;

Jones, R.D.; Scott, T.R.

Opt. Quant. Electron. 26(1994): 25-34; Jan 94

Electrically Calibrated Pyroelectric Detector-Refinements for Improved Optical Power Measurements;

Phelan, R.J., Jr.; Lehman, J.H.; Larson, D.R.

Proc., SPIE, Vol 2022, Tech. Conf., Photodetectors and Power Meters, Jul 1516, 1993, San Diego, CA: 160-164; 93

Optical Density Measurement of Laser Eye Protection Materials;

Scott, T.R.; Rockwell, R.J., Jr.; Batra, P.

Proc., 1992 Intl. Laser Safety Conf., Dec 1-4, 1992, Cincinnati, OH, Section 8-7 to $8-16 ; 93$

International Intercomparison of Detector Responsivity at 1300 and $1550 \mathrm{~nm}$;

Gallawa, R.L.; Gardner, J.L.; Nettleton, D.H.; Stock, K.D.

Proc., Conf. on Prec. Electromagn. Meas., '92, 268-269; Jan 93

Laser-Beam Analysis Pinpoints Critical Parameters;

Jones, R.D.; Scott, T.R.

Laser Focus World 29(1): 123-130; Jan 93

Beam Analysis Round Robin;

Jones, R.D.; Scott, T.R.

Proc., SPIE, Vol 1834: 60-71; 92 
Laser Radiometry

International Intercomparison of Detector Responsivity at 1300 and $1550 \mathrm{~nm}$;

Gardner, J.L.; Gallawa, R.L.; Stock, K.D.; Nettleton, D.H. Appl. Opt. 31(34): 7226-7231; Dec 1, 92

International Intercomparison of Detector Responsivity at 1300 and $1550 \mathrm{~nm}$;

Gallawa, R.L.; Gardner, J.L.; Nettleton, D.H.; Stock, K.D.

Proc., Conf. on Prec. Electromagn. Meas., 268-269; Jun 92

Spatial Uniformity Measurement of Optical Detector Response;

Livigni, D.J.; Li, X.

Proc., '92 Measurement Science Conf.; Jan 92

Megawatt Laser Calorimeter Design;

Scott, T.R.

Tech. Dig., IEEE Instrum. Meas. Conf., 227-231; 91

A Limited International Intercomparison of Responsivity Scales at Fiber Optic Wavelengths;

Gallawa, R.L.; Li, X.; Gardner, J.L.; Nettleton, D.H.; Stock, K.D.; Ward, T.H. NIST JRES 96(2): 225-230; Mar-Apr, 91

A Low Temperature Electrically Calibrated SOS Bolometer for Power and Energy Measurements;

Craig, R.M.; Phelan, R.J., Jr.

Proc., 1990 Measurement Science Conf., Feb 8-9, 1990, Anaheim, CA; 90

Hydrogenated Amorphous Germanium Detectors Deposited onto Channel Waveguides;

Phelan, R.J., Jr.; Larson, D.R.

Opt. Lett. 15(10): 544-546; May 15, 90

Scanning System for Measuring Uniformity of Laser Detector Response and Laser Beam Dimensions;

Rasmussen, A.L.; Case, W.E.; Sanders A.A.

NISTIR 90-3937, 100 pp; Apr 90

NIST Optical Power Measurements;

Scott, T.R.

Proc., 1989 Measurement Science Conf., Jan 26-27, 1989: 3C-19 to 3C-29; 89

Potential Errors in the Use of Optical Fiber Power Meters;

Li, X.; Gallawa, R.L.

Proc., SPIE, Vol 841: 231-233; 88 
Review of NBS Laser Power and Energy Measurements;

Scott, T.R.

Proc., SPIE, Vol 888: 48-54; 88

System for Measuring Optical Waveguide Intensity Profiles;

Larson, L.E.; Larson, D.R.; Phelan, R.J., Jr.

NBSIR 88-3092, 68 pp; Oct 3, 88

A Single Launch Technique for Determination of Mode Transfer Matrices;

Yang, S.; Hjelme, D.R.; Januar, I.P; Vayshenker, I.; Mickelson, A.R.

Tech. Dig., Optical Fiber Meas. Symp., in NIST SP 748, 103-106; Sep 88

NBS Standards for Optical Power Meter Calibration;

Scott, T.R.

Proc., DoD/ANSI/EIA Fiber Optics Standardization Symp., 224-238; 87

On the Calibration of Optical Fiber Power Meters: The Effect of Connectors;

Gallawa, R.L.; Li, X.

Appl. Opt. 26(7): 1170-1174; Apr 87

Optical Power Meters: A Round Robin Test of Uncertainty;

Gallawa, R.L.; Yang, S.

Appl. Opt. 25(7): 1066-1068; Apr 86

Laser Power and Energy Measurements and the NBS Laser Measurement Assurance Program (MAP);

Case, W.E.; Sanders, A.A.

IEEE Instrumentation and Measurement Technology Conf., Mar 20-22, 1985, Tampa, FL, 281-285; 85

Laser Measurements;

Sanders, A.A.

Proc., 1983 Measurement Science Conf., Jan 20-21, 1983, Palo Alto, CA; Jun 83

Beam-Profile Measurement of Pulses Using a Spatial Filter to Sample the Hermite Modes for a String of Pulses;

Johnson, E.G., Jr.

NBS TN 1057; Sep 82 
Laser Radiometry

Documentation of the NBS C K and Q Laser Calibration Systems;

Case, W.E.

NBSIR 82-1676; Sep 82

The Use of LEDs as YAG Laser Simulators;

Young, $\mathrm{M}$.

Proc., Conf. on Electro-Optics and Lasers; Nov 81

The Use of LEDs to Simulate Weak YAG-Laser Beams;

Young, $\mathrm{M}$.

NBS TN 1031, revised; Aug 81

Continuous-Wave (Mode-Locked) Dye Laser with Unfolded Cavity;

Young, $\mathrm{M}$.

Appl. Opt. 18: 3212; Oct 79

National Standards of a Powerful Sort;

Sanders, A.A.

Opt. Spectra 13: 45; Aug 79

Quality Assurance Program for the NBS C K and Q Laser Calibration Systems;

Case, W.E.

NBSIR 79-1619; Aug 79

Design of a Reflection Apparatus for Laser Beam Profile Measurements;

Johnson, E.G., Jr.

NBS TN 1015; Jul 79

Laser Beam Profile Measurements Using Spatial Sampling Fourier Optics and Holography;

Johnson, E.G., Jr. NBS TN 1009; Jan 79

Improvements in a Calorimeter for High-Power CW Lasers;

Chamberlain, G.E.; Simpson, P.A.; Smith, R.L.

IEEE Trans. Instrum. Meas. IM-27: 81-86; Mar 78

Laser Far-Field Beam-Profile Measurements by the Focal Plane Technique;

Day, G.W.; Stubenrauch, C.F.

NBS TN 1001; Mar 78 
Evaluating the Inequivalence and a Computational Simplification for the NBS Laser Energy Standards;

Johnson, E.G., Jr.

Appl. Opt. 16: 2315-2321; Aug 77

Measurement Procedures for the Optical Beam Splitter Attenuation Device BA-1;

Danielson, B.L.

NBSIR 77-858; May 77

An NBS Laser Measurement Assurance Program (MAP);

Sanders, A.A.; Cook, A.R.

Proc., Electro-Optical Systems Design Conf. 1976 and Intl. Laser Exposition, Sep 14-16, 1976, New York, NY, 277-280; 76

Laser Action in Sputtered Metal Vapors;

McNeil, J.R.; Johnson, W.L.; Collins, G.J.; Persson, K.B.; Franzen, D.L. 9th Intl. Conf. on Quantum Electronics, Jun 14-16, 1976, Amsterdam, The Netherlands, 162-163; 76

Performance and Characteristics of Polyvinylidene Fluoride Pyroelectric Detectors;

Day, G.W.; Hamilton, C.A.; Gruzensky, P.M.; Phelan, R.J., Jr.

Proc., IEEE Symposium on Application of Ferroelectrics, Jun 9-11, 1975, Albuquerque, NM, Ferroelectrics, Vol 10: 99-102; 76

Spectral Reference Detector for the Visible to 12 Micrometer Region Convenient Spectrally Flat;

Day, G.W.; Hamilton, C.A.; Pyatt, K.W.

Appl. Opt. 15: 1865-1868; Jul 76

An Electrically Calibrated Pyroelectric Radiometer System;

Hamilton, C.A.; Day, G.W.; Phelan, R.J., Jr.

NBS TN 678; Mar 76

Laser Attenuators for the Production of Low Power Beams in the Visible and 1.06 Micron Regions;

Danielson, B.L.; Beers, Y.

NBS TN 677; Jan 76

Radiometry without Standard Sources/Electrically Calibrated Pyroelectrics;

Phelan, R.J., Jr.; Hamilton, C.A.; Day, G.W.

Proc., SPIE, Vol 62: 159-165; 75 
Laser Radiometry

Pyroelectric Radiometers Get Off the Drawing Board;

Hamilton, C.A.; Phelan, R.J., Jr.; Day, G.W.

Opt. Spectra 9: 37-38; Oct 75

Improving Beam Measurement;

Smith, R.L.; Sanders, A.A.

Laser Focus 70-71; Apr 75

Precision Beam Splitters for $\mathrm{CO}_{2}$ Lasers;

Franzen, D.L.

Appl. Opt. 14: 647-652; Mar 75

A Pyroelectric Power Meter for the Measurement of Low Level Laser Radiation;

Hamilton, C.A.; Day, G.W.

NBS TN 665; Feb 75

The Polarization of PVF and $\mathrm{PVF}_{2}$ Pyroelectrics;

Phelan, R.J., Jr.; Peterson, R.L.; Hamilton, C.A.; Day, G.W.

Ferroelectrics 7: 375-377; 74

Current Status of NBS Low-Power Laser Energy Measurement;

West, E.D.; Case, W.E.

Proc., Conf. on Prec. Electromagn. Meas., Jul 1-5, 1974, London, England,

IEEE Trans. Instrum. Meas. IM-23: 422-425; Dec 74

Analysis of Response of Pyroelectric Optical Detectors;

Peterson, R.L.; Day, G.W.; Gruzensky, P.M.; Phelan, R.J., Jr.

J. Appl. Phys. 45: 3296-3303; Aug 74

Effects of Poling Conditions on Responsivity and Uniformity of Polarization of $\mathrm{PVF}_{2}$ Pyroelectric Detectors;

Day, G.W.; Hamilton, C.A.; Peterson, R.L.; Phelan, R.J., Jr.; Mullen, L.O. Appl. Phys. Lett. 24: 456-458; May 74

Absolute Pyroelectric Radiometers and Two Dimensional Arrays;

Phelan, R.J., Jr.; Peterson, R.L.; Klein, G.P.; Hamilton, C.A.; Day, G.W. Proc., American Electro-Optical Systems Design Conf., New York, NY, $117-123 ; 73$ 
Comparison of the Laser Power and Total Irradiance Scales Maintained by the National Bureau of Standards;

Geist, J.; Schmidt, L.B.; Case, W.E.

Appl. Opt. 12: 2773-2776; Nov 73

Electrically Calibrated Pyroelectric Optical-Radiation Detector;

Phelan, R.J., Jr.; Cook, A.R.

Appl. Opt. 10: 2494-2500; Oct 73

Continuous Laser-Sustained Plasmas;

Franzen, D.L.

J. Appl. Phys. 44: 1727-1732; Apr 73

Limitations of the Use of Vacuum Photodiodes in Instruments for the Measurement of Laser Power and Energy;

Smith, R.L.; Phelan, R.J., Jr.

Appl. Opt. 12: 795-798; Apr 73

Accurate Frequencies of Molecular Transitions Used in Laser Stabilization: The

3.39-Micrometer Transition in $\mathrm{CH} 4$ and the 9.33- and 10.18-Micrometer Transitions in $\mathrm{CO}_{2}$;

Evenson, K.M.; Wells, J.S.; Petersen, F.R.; Danielson, B.L.; Day, G.W. Appl. Phys. Lett. 22: 192-195; Feb 73

A Calorimeter for High Power CW Lasers;

Smith, R.L.; Case, W.E.; Rasmussen, A.L.; Russell, T.W.; West, E.D.

Proc., Conf. on Prec. Electromagn. Meas., Jun 26-29, 1972, Boulder, CO, $138-139 ; 72$

High D* Fast Lead Zirconate Titanate Pyroelectric Detectors;

Mahler, R.J.; Phelan, R.J., Jr.; Cook, A.R.

Infrared Phys. 12: 57-59; 72

A Calorimeter for High-Power CW Lasers;

Smith, R.L.; Russell, T.W.; Case, W.E.; Rasmussen, A.L.

IEEE Trans. Instrum. Meas. IM-21: 434-438; Nov 72

CW Gas Breakdown in Argon Using 10.6 Micrometer Laser Radiation;

Franzen, D.L.

Appl. Phys. Lett. 21: 62-64; Jul 72 
Laser Radiometry

Role of Infrared Frequency Synthesis in Metrology;

Wells, J.S.; Evenson, K.M.; Day, G.W.; Halford, D.

Proc., IEEE Lett., Vol 60: 621-623; May 72

Double Plate Calorimeter for Measuring the Reflectivity of the Plates and the Energy in a Beam of Radiation;

Rasmussen, A.L.

PATENT-3 622, 245; patented; Nov 23, 71

High D* Pyroelectric Polyvinylfluoride Detectors;

Phelan, R.J., Jr.; Mahler, R.J.; Cook, A.R.

Appl. Phys. Lett. 19: 337-338; Nov 71

Measurement of Laser Energy of Linear Components of Polarization at 1.060 Micron;

Rasmussen, A.L.

Rev. Sci. Instrum. 42: 1590-1593; Nov 71

Extension of Absolute Frequency Measurements to the cw He-Ne Laser at $88 \mathrm{THz}(3.39 \mu \mathrm{m})$; Evenson, K.M.; Day, G.W.; Wells, J.S.; Mullen, L.O.

Appl. Phys. Lett. 20: 133-134; Feb 71

Laser Power and Energy Measurements;

Jennings, D.A.; West, E.D.; Evenson, K.M.; Rasmussen, A.L.; Simmons, W.R. NBS TN 382; Oct 69 


\section{OPTICAL FIBER METROLOGY}

Mode-Field Diameter of Single-Mode Optical Fiber by Far-Field Scanning: Addendum; Young, $M$.

Appl. Opt. 37(36): 8361; Dec 20, 98

Zero-Dispersion Wavelength Distribution in Optical Fibers from CW Four-Wave Mixing; Schlager, J.B.

Proc., IEEE Lasers and Electro-Optics Society, 11th Annual Mtg., Vol 2, Dec 3-4, 1998, Orlando, FL, 309-310; Dec 98

Are the Formulas for Mode-Field Diameter Correct?;

Wittmann, R.C.; Young, M.

Tech. Dig., Symp. on Optical Fiber Meas., in NIST SP 930, 69-72; Sep 98

Biased Perturbation Method for Index Profiling Single-Mode Fibers from Near-Field and FarField Data;

Fontaine, N.H.; Young, M.

Tech. Dig., Symp. on Optical Fiber Meas., in NIST SP 930, 61-64; Sep 98

Differential Mode Delay Measurements on Multimode Fibers in the Time and Frequency Domains;

Schlager, J.B.; Franzen, D.L.

Tech. Dig., Symp. on Optical Fiber Meas., in NIST SP 930, 127-130; Sep 98

Narrowband Measurements of Polarization-Mode Dispersion Using the Modulation Phase Shift Technique;

Williams, P.A.; Barlow, A.J.; Mackechnie, C.; Schlager, J.B.

Tech. Dig., Symp. on Optical Fiber Meas., in NIST SP 930, 23-26; Sep 98

Mode-Field Diameter of Single-Mode Optical Fiber by Far-Field Scanning;

Young, $\mathrm{M}$.

Appl. Opt. 37(24): 5605-5619; Aug 20, 98

Corrections to Fixed Analyzer Measurements of Polarization Mode Dispersion;

Williams, P.A.; Wang, C.-M.

J. Lightwave Tech. 16(4): 534-541; Apr 98

Optical Fiber Connectors: An Interlaboratory Comparison of Measurements of Endface Geometry;

Drapela, T.J.

NIST TN 1503, 32 pp; Apr 98 
Optical Fiber Metrology

Preliminary Experiments on Coherent Optical Code Division Multiple Access Using ArrayedWaveguide Grating Pairs;

Takiguchi, K.; Okamoto, K.; Ohmori, Y.; Mechels, S.

Proc., 1998 IEICE General Conf., March 27-30, 1998, Hiratsuka, Japan, 281;

Mar 98

International Comparison: Zero-Dispersion Wavelength in Single-Mode Optical Fibres

(Wavelength); Final Report;

Mechels, S.E.

Metrologia 34: 449; 97

Bibliography of NIST Publications on Multimode Optical Fibers;

Metz, S.E., Young, M., eds.

NISTIR 5070; 23 pp; Dec 97

High Resolution Differential Mode Delay and Bandwidth Measurements in Multimode Fibers; Schlager, J.B.; Mechels, S.E.; Franzen, D.L.

Proc., IEEE Lasers and Electro-Optics Society, 10th Annual Mtg., Vol 1, Nov 10-13, 1997, San Francisco, CA, 46-47; Nov 97

Differential Mode Delay Measurements in Multimode Fibers Using a Frequency Domain Technique with Variable Launch;

Franzen, D.L.; Mechels, S.E.; Schlager, J.B.

Conf. Dig., OFMC'97, 4th Optical Fibre Measurement Conf., Sep 29-Oct 1, 1997, Teddington, UK, 109-112; Oct 1, 97

Rotating-Polarizer Polarimeter for Accurate Retardance Measurement;

Williams, P.A.; Rose, A.H.; Wang, C.M.

Appl. Opt. 36(25): 6466-6472; Sep 1, 97

Accurate Measurements of the Zero-Dispersion Wavelength in Optical Fibers;

Mechels. S.E.: Schlager, J.B.; Franzen, D.L.

NIST JRES 102(3): 333-347; May-Jun, 97

High-Resolution Differential-Mode Delay Measurements in Optical Fibers Using a Frequency-

Domain Phase-Shift Technique;

Mechels, S.E.; Schlager, J.B.; Franzen, D.L.

IEEE Photonics Tech. Lett. 9(6): 794-796; Jun 97

Robust Regression Applied to Optical-Fiber Dimensional Quality Control;

Wang, C.-M.; Vecchia, D.F.; Young, M.; Brilliant, N.A.

Technometrics 39(1): 25-33; Feb 97 
Lightwave Metrology: Optical Fibers and Power Meters;

Danielson, B.L.; Drapela, T.J.; Franzen, D.L.; Mechels, S.; Scott, T.R.; Vayshenker, I.; Young, M.

URSI, Review of Radio Science 1993-1996: W. Ross Stone, ed., Chapter 5, $71-113 ; 96$

Accuracy in PMD Measurements;

Williams, P.A.

Proc., IEEE Lasers and Electro-Optics Society, 9th Annual Mtg., Vol 1, Nov 18-19, 1996, Boston, MA, 170-171; Nov 96

Zero-Dispersion Wavelength Uniformity and Four-Wave Mixing in Optical Fiber;

Schlager, J.B.; Mechels, S.; Franzen, D.L.

Proc., IEEE Lasers and Electro-Optics Society, 9th Annual Mtg., Vol 1, Nov 18-19, 1996, Boston, MA, 166-167; Nov 96

Accuracy Issues in Comparisons of Time- and Frequency-Domain Polarization Mode

Dispersion Measurements;

Williams, P.A.

Tech. Dig., Symp. on Optical Fiber Meas., in NIST SP 905, 125-130; Oct 96

Determination of Zero-Dispersion Wavelength in Optical Fiber Using Four-Wave Mixing;

Schlager, J.B.; Mechels, S.; Franzen, D.L.

Tech. Dig., Symp. on Optical Fiber Meas., in NIST SP 905, 121-124; Oct 96

TIA Round Robin for the Measurement of PMD;

Williams, P.A.

Tech. Dig., Symp. on Optical Fiber Meas., in NIST SP 905, 155-158; Oct 96

Precise Laser-Based Measurements of Zero-Dispersion Wavelength in Single-Mode Fibers;

Schlager, J.B.; Mechels, S.; Franzen, D.L.

Tech. Dig., Optical Fiber Communication Conf. (OFC '96), Feb 25-Mar 1, 1996, San Jose, CA, Vol 6: 293-294; Mar 96

Anomalous Relation Between Time and Frequency Domain PMD Measurements;

Williams, P.A.; Hernday, P.R.

Tech. Prog., OFMC'95, 3rd Optical Fibre Measurement Conf., Sep 25-26, 1995, Liege, Belgium: Talk Section I-2; 95 
Optical Fiber Metrology

Standard Reference Materials for Optical Fibers and Connectors;

Young, $\mathrm{M}$.

Tech. Dig., Optical Fiber Communication Conf. (OFC'95), Feb 26-Mar 3, 1995, San Diego, CA, Vol 8: 239-240; 95

Off-Axis Illumination and its Relation to Partial Coherence;

Young, M.; Hale, P.D.

Am. J. Phys. 63(12): 1136-1141; Dec 95

Accurate Zero-Dispersion Wavelength Measurements in Single-Mode Fibers: Two FrequencyDomain Methods;

Mechels, S.E.; Schlager, J.B.; Franzen, D.L.

Proc., IEEE Lasers and Electro-Optics Society, 8th Annual Mtg, Vol 2, Nov 12, 1995, San Francisco, CA, 75-76; Nov 95

Optical Fiber, Fiber Coating, and Connector Ferrule Geometry: Results of Interlaboratory Measurement Comparisons;

Drapela, T.J.; Franzen, D.L.; Young, M. NIST TN 1378, 61 pp; Nov 95

Cladding Standard Improves Fiberoptic Measurements;

Young, $\mathrm{M}$.

Laser Focus World, 31(10): 26-32; Oct 95

Accurate Measurements of Zero-Dispersion Wavelength in Single-Mode Fibers;

Mechels, S.; Franzen, D.L.

Tech. Prog., OFMC'95, 3rd Optical Fibre Measurement Conf., Sep 25-26, 1995, Liege, Belgium; Sep 25, 95

Fiber Coating Diameter: Toward a Glass Artifact Standard;

Williams, D.H.; Young, M.; Tietz, L.A.

Tech. Prog., OFMC'95, 3rd Optical Fibre Measurement Conf., Sep 25-26, 1995, Liege, Belgium III.2; Sep 95

Vector and Quasi-Vector Solutions for Optical Waveguide Modes Using Efficient Galerkin's Method with Hermite-Gauss Basis Functions;

Weisshaar, A.; Li, J.; Gallawa, R.L.; Goyal, I.C.

J. Lightwave Tech. 13(8): 1795-1800; Aug 95 
Millimeter-Resolution Optical Time-Domain Reflectometry Using a Four Wave Mixing Sampling Gate;

Schlager, J.B.; Jinno, M.; Franzen, D.L. Photonic Tech. Lett. 7(2): 206-208; Feb 95

Fiber Splice Loss: A Simple Method of Calculation;

Gallawa, R.L.; Kumar, A.; Weisshaar, A.

Opt. Quant. Electron. 26: S165-S172; 94

Optical Sampling Using Nondegenerate Four-Wave Mixing in a Semiconductor Laser Amplifier;

Jinno, M.; Schlager, J.B.; Franzen, D.L.

Tech. Dig., Opt. Soc. Am., Optical Amplifiers and Their Applications Topical Mtg., Vol 14, Aug 3-5, 1994, Breckenridge, CO, 147-149; 94

Calculated Fiber Attenuation: A General Method Yielding Stationary Values;

Gallawa, R.L.; Goyal, I.C.; Ghatak, A.K.

J. Lightwave Tech. LT-11(12): 1900-1904; Dec 94

Software for Performing Gray-Scale Measurements of Optical Fiber End Faces;

Wang, C.-M.; Vecchia, D.F.; Young, M.; Brilliant, N.A.

NIST TN 1370; Nov 94

Video Microscopy Applied to Optical Fiber Geometry Measurements;

Brilliant, N.A.; Alpert, B.K.; Young, M.

NIST TN 1369; Nov 94

Optical Sampling Using Nondegenerate Four-Wave Mixing in a Semiconductor Laser Amplifier;

Jinno, M.; Schlager, J.B.; Franzen, D.L.

Electron. Lett. 30(18): 1489-1490; Sep 1, 94

Fiber Geometry: Results of an International Interlaboratory Measurement Comparison;

Drapela, T.J.; Franzen, D.L.; Young, M.

Tech. Dig., Optical Fiber Meas. Symp., in NIST SP 864, 129-132; Sep 94

Lightwave Standards Development at NIST;

Franzen, D.L.

Proc., DoD Fiber Optics Conf. '94, Optical Networks in the Concept of a Global Grid, Mar 22-24, 1994, McLean, VA, 443-445; Mar 94 
Optical Fiber Metrology

Low-Coherence Interferometric Measurement of Group Transit Times in Precision Optical Fiber Delay Lines;

Danielson, B.L.

Tech. Dig., 2nd Optical Fibre Meas. Conf., Sep 21-22, 1993, Torino, Italy, $159-162 ; 93$

Outlier-Resistant Fitting of Gray-Scale Images Illustrated by Optical Fiber Geometry;

Vecchia, D.F.; Wang, C.-M.; Young, M.

Proc., 1993 Meas. Sci. Conf., Jan 21-22, 1993, Los Angeles, CA, , 1-10; 93

High-Sensitivity Optical Sampling Using an Erbium-Doped Fiber Laser Strobe;

Schlager, J.B.; Hale, P.D.; Franzen, D.L.

Microwave Opt. Tech. Lett. 6(15): 835-837; Dec 5, 93

Vector Theory of Diffraction by a Single-Mode Fiber: Application to Mode-Field Diameter Measurements;

Young, M.; Wittmann, R.C.

Opt. Lett. 18(20): 1715-1717; Oct 15, 93

Conference Report: Symposium on Optical Fiber Measurements;

Franzen, D.L.; Day, G.W.

NIST JRES 98(2): 253-255; Mar-Apr 93

Optical Fiber Geometry: Accurate Measurement of Cladding Diameter;

Young, M.; Hale, P.D.; Mechels, S.E.

NIST JRES 98(2): 203-216; Mar-Apr, 93

Fiber Spot Size: A Simple Method of Calculation;

Gallawa, R.L.; Goyal, I.C.; Ghatak, A.K.

J. Lightwave Tech. LT-11(2): 192-197; Feb 93

Pump-Induced Dispersion of Erbium-Doped Fiber Measured by Fourier-Transform Spectroscopy;

Hickernell, R.K.; Yamada, M.; Shimizu, M.; Horiguchi, M.

Opt. Lett. 18(1): 19-21; Jan 1, 93

Optical Fiber Geometry: Accurate Measurement of Cladding Diameter;

Young, M.; Mechels, S.E.; Hale, P.D.

Proc., Conf. on Prec. Electromagn. Meas. '92, 202-203; Jan 93 
Transfer Functions for Characterizing Multimode Optical Fiber Components;

Yang, $S$.

NISTIR 3997, 132 pp; Jan 93

Anharmonic Oscillator Analysis Using Modified Airy Functions;

Goyal, I.C.; Roy, S.; Ghatak, A.K.; Gallawa, R.L.

Can. J. Phys. 70: 1218-1221; 92

Fiber Cladding Diameter Measurement by White Light Interference Microscopy;

Hale, P.D.; Franzen, D.L.

NIST SP $839 ; 92$

Multiwavelength Birefringent-Cavity Mode-Locked Fiber Laser;

Takara, H.; Kawanishi, S.; Saruwatari, M.; Schlager, J.B.

Tech. Dig., Conf. on Lasers and Electro-Optics (CLEO' 92), May 10-15, 1992, Anaheim, CA, 250-251; 92

Overview of Photonic Primary Standards Development;

Franzen, D.L.

Dig. '92, DoD Fiber Optic Conf., 357-358; 92

Scanning Confocal Microscope for Accurate Dimensional Measurement;

Mechels, S.E.; Young, M.

Proc., SPIE, Vol 1660, Biomedical Image Processing and Three-Dimensional

Microscopy: 542-550; 92

Multi-Wavelength Birefringent-Cavity Mode-Locked Fibre Laser;

Takara, H.; Kawanishi, S.; Saruwatari, M.; Schlager, J.B.

Electron. Lett. 28(25): 2274-2275; Dec 3, 92

Accurate Measurements of Fiber Cladding Diameter;

Young, M.; Mechels, S.E.; Hale, P.D.

Tech. Dig., Optical Fiber Meas. Symp., in NIST SP 839, 55-58; Sep 92

Fiber Cladding Diameter Measurement by White Light Interference Microscopy;

Hale, P.D.; Franzen, D.L.

Tech. Dig., Optical Fiber Meas. Symp., in NIST SP 839, 51-54; Sep 92

Single-Mode Fiber Geometry and Chromatic Dispersion: Results of Interlaboratory Comparisons;

Drapela, T.J.; Franzen, D.L.; Young, M.

Tech. Dig., Optical Fiber Meas. Symp., in NIST SP 839, 187-190; Sep 92 
Optical Fiber Metrology

Optical Fiber Geometry by Gray-Scale Analysis with Robust Regression;

Mamileti, L.; Young, M.; Wang, C.-M.; Vecchia, D.F.

Appl. Opt. 31(21): 4182-4185; Jul 20, 92

Metrology Applications of Mode-Locked Erbium Fiber Lasers;

Schlager, J.B.; Hale, P.D.; Franzen, D.L.

Proc., Conf. on Prec. Electromagn. Meas. '92, 261; Jun 92

Optical Fiber Geometry: Accurate Measurement of Cladding Diameter;

Young, M.; Mechels, S.E.; Hale, P.D.

Conf. Prec., Electromagn. Meas. '92, 202-203; Jun 92

$\mathrm{LP}_{11}$-Mode Leakage Loss in Coated Depressed Clad Fibers;

Pal, B.P.; Gallawa, R.L.; Goyal, I.C.

Photon. Tech. Lett. 4(4): 376-378; Apr 92

Dispersion-Shifted Dual-Shape Core Fibers: Optimization Based on Spot-Size Definitions;

Tewari, R.; Pal, B.P.; Das, U.K.

J. Lightwave Tech. LT-10(1): 1-5; Jan 92

Scanning Confocal Microscopy for Measuring Diameter and Linewidth: Numerical

Modelling;

Obarski, G.E.; Drapela, T.J.; Young, M.

Proc., SPIE, Vol 1640: 761-772; Jan 92

Fiber Cladding Diameter by Contact Micrometry;

Young, $\mathbf{M}$.

Proc.. Optical Fibre Meas. Conf., Sep 17-18, 1991, York, U.K., 123-126; 91

Scanning Confocal Microscope for Precise Measurements of Optical Fiber Diameter;

Mechels, S.E.: Young, $M$.

Proc.. SPIE, Vol 1556: 164-170; 91

Video Microscope with Sub-Micrometer Resolution;

Mechels. S.E.: Young, M.

Appl. Opt. 30: 2202-2211; 91

Modified Airy Function and WKB Solutions to the Wave Equation;

Ghatak, A.K.; Gallawa, R.L.; Goyal, I.C.

NIST MN 176; Nov 91 
Absolute Optical Ranging Using Low Coherence Interferometry;

Danielson, B.L.; Boisrobert, C.Y. Appl. Opt. 30(21): 2975-2979; Jul 20, 91

Symposium on Optical Fiber Measurements;

Day, G.W.; Franzen, D.L.

NIST JRES 96(2): 231-232; Mar-Apr, 91

Measurement Standards to Support Photonics Technology;

Franzen, D.L.

Proc., IEEE Instrum. Meas. Tech. Conf., Feb 13-15, 1990, San Jose, CA, 326-328; 90

Recirculating Pulse Erbium-Fiber Ring Amplifier;

Schlager, J.B.; Yamabayashi, Y.; Franzen, D.L.

Proc., Optical Fiber Communication Conf. (OFC'90), Jan 22-26, 1990, San

Francisco, CA; 90

Analyzing Integrated Optical Waveguides: A Comparison of Two New Methods;

Tu, Y.; Goyal, I.C.; Gallawa, R.L.

Appl. Opt. 29(36): 5313-5315; Dec 20, 90

Standards for Optical Fiber Geometry Measurements;

Young, $\mathrm{M}$.

NIST SP 792; Sep 90

Subpicosecond Pulse Compression and Raman Generation Using a Mode-Locked

Erbium-Doped Laser Amplifier;

Schlager, J.B.; Hale, P.D.; Franzen, D.L.

Photonics Tech. Lett. 2(8): 562-564; Aug 90

Soliton-Like Compression of Pulses from Erbium-Fiber Lasers;

Schlager, J.B.; Yamabayashi, Y.; Franzen, D.L.

Proc., European Conf. on Optical Communications, ECOC'89, Sep 10-14, $1989,62-65 ; 89$

Mode-Locked Long-Cavity Erbium Fiber Lasers with Subsequent Soliton-Like Compression; Schlager, J.B.; Yamabayashi, Y.; Franzen, D.L.; Juneau, R.I.

Photonics Tech. Lett. 1(9): 264-266; Sep 89 
Optical Fiber Metrology

A Comparison of Far-Field Methods for Determining Mode Field Diameter of Single-Mode Fibers Using Both Gaussian and Petermann Definitions;

Drapela, T.J.; Franzen, D.L.; Cherin, A.H.; Smith, R.J.

J. Lightwave Tech. 7(8): 1153-1157; Aug 89

Numerical Aperture of Multimode Fibers by Several Methods: Resolving Differences;

Franzen, D.L.; Young, M.; Cherin, A.H.; Head, E.D.; Hackert, M.; Raine, K.;

Baines, J.

J. Lightwave Tech. LT- 7(6): 896-910; Jun 89

A Standard Reference Fiber for Calibration of Optical Time Domain Reflectometers;

Danielson, B.L.; Whittenberg, C.; Drapela, T.J.

Tech. Dig., 35th Intl. Instrumentation Symp., Paper 4.6; May 89

Spatial Filtering Microscope for Linewidth Measurements;

Young, $M$.

Appl. Opt. 28(8): 1467-1473; Apr 15, 89

Profile Inhomogeneity in Multimode Graded-Index Fibers;

Oates, C.W.; Young, M.

J. Lightwave Tech. LT-7(3): 530-532; Mar 89

Measurement Standards for Single-Mode Fibers;

Franzen, D.L.

Proc., 1988 Conf. on Prec. Electromagn. Meas., 121-122; 88

Optical Fiber Measurements: Results of Interlaboratory Evaluations;

Franzen, D.L.

Proc., SPIE, Vol 992: 242-244; 88

An Electronic Industries Association Interlaboratory Comparison to Resolve Differences in Multimode Fiber Numerical Aperture Measurements;

Cherin, A.H.; Head, E.; Franzen, D.L.; Young, M.; Hackert, M.

Tech. Dig., Optical Fiber Meas. Symp., in NIST SP 748, 157-160; Sep 88

Interlaboratory Comparison of Far-Field Methods for Determining Mode Field Diameter Using Both Gaussian and Petermann Definitions;

Drapela, T.J.

NBS SP 748, 179-182; Sep 88 
Group Index and Time Delay Measurements of a Standard Reference Fiber;

Danielson, B.L.; Whittenberg, C.D.

NBSIR 88-3091, 20 pp; Jul 88

Interferometric Dispersion Measurements on Small Guided-Wave Structures;

Danielson, B.L.; Whittenberg, C.

Tech. Dig., Conf. on Lasers and Electro-Optics (CLEO '88), pages unnumbered; Apr 88

Conference Report: Symposium on Optical Fiber Measurements;

Day, G.W.; Franzen, D.L.

NBS JRES Vol 92: 69-70; 87

Optical Fiber Metrology at the National Bureau of Standards;

Franzen, D.L.

Proc., DoD/ANSI/EIA Fiber Optics Standardization Symp., 222-223; 87

Calibration and Standardization Issues for the Optical Time-Domain Reflectometer; Danielson, B.L.

NBSIR 87-3078, 16 pp; Dec 87

Guided-Wave Reflectometry with Micrometer Resolution;

Danielson, B.L.; Whittenberg, C.D.

Appl. Opt. 26(14): 2836-2842; Jul 15, 87

Image Processing Software for Optical Engineering;

Weppner, M.B.; Young, M.

NBSIR 87-3065; Apr 87

Optical Sampling with Gain-Switched Pulse Compressed Distributed-Feedback Laser Diodes;

Franzen, D.L.; Yamabayashi, Y.; Kanada, T.

Electron. Lett. 23(6): 289-290; Mar 87

Attenuation Measurements on Deformed Optical Fibers;

Engelsrath, A.; Danielson, B.L.; Franzen, D.L.

NBSIR 86-3052, 28 pp; 86

Measurement Procedures for Optical Fiber and Related Components;

Danielson, B.L.; Day, G.W.; Franzen, D.L.; Gallawa, R.L.; Kim, E.; Phelan, R.J., Jr.; Young, $M$.

Rome AFB NY, RADC-TR-86-81; Aug 86 
Optical Fiber Metrology

A Comparison of Three Bandwidth Measurements Techniques for Optical Fibers; Shao, Y.; Gallawa, R.L.

IEEE Trans. Instrum. Meas. IM-35(2): 187-194; Jun 86

Single Mode Fiber Dispersion Measurements Using Optical Sampling with a Modelocked Laser Diode;

Kanada, T.; Franzen, D.L.

Opt. Lett. 11: 330-332; May 86

Standard Measurement Procedures for Characterizing Single-Mode Fiber;

Franzen, D.L.

Conf. Dig., Test and Measurement World Expo., San Jose, CA; Apr 86

Optical Waveform Measurement by Optical Sampling/Cross Correlation with a Mode Locked Laser Diode;

Kanada, T.; Franzen, D.L.

Opt. Lett. 11(1): 4-6; Jan 86

Interlaboratory Measurement Comparison among Fiber Manufacturers to Determine the

Effective Cutoff Wavelength and Mode-Field Diameter of Single-Mode Fiber;

Franzen, D.L.

Tech. Dig., Optical Fiber Communication Conf., Feb 11-13, 1985, San Diego, CA, 36; 85

Introduction to Fiber Optics: Short-Haul and Long-Haul Measurements and Applications III;

Gallawa, R.L.

Proc., SPIE, Vol 599: vi; 85

Pulse Spectrum Analysis Method of Measuring Fiber Bandwidth;

Yang, S.; Alvarez, R.; Weimer, C.; Gallawa, R.L.

Proc., SPIE, Vol 559: 207-210; 85

Some Issues in Optical Fiber Bandwidth Measurement;

Yang, S.; Gallawa, R.L.

Proc., IEEE Instrumentation and Measurement Conf., Mar 20-22, 1985, Tampa, FL, 228; 85

Use of Mode Transfer Matrices in L.A.N. Loss Evaluation; Maisonneuve, J.M.; Churoux, P.; Gallawa, R.L.

Proc., SPIE, Vol 559: 182-185; 85 
Determining the Mode-Field Diameter of Single-Mode Optical Fiber: An Interlaboratory Comparison;

Franzen, D.L.; Srivastava, R.

J. Lightwave Tech. LT-3(5): 1073-1077; Oct 85

Optical Time-Domain Reflectometer Specifications and Performance Testing;

Danielson, B.L.

Appl. Opt. 24(15): 2313-2322; Aug 1, 85

Determining the Effective Cutoff Wavelength of Single-Mode Fibers: An Interlaboratory Comparison;

Franzen, D.L.

J. Lightwave Tech. LT-3(1): 128-133; Feb 85

Intramodal Part of the Transfer Function for an Optical Fiber;

Rodhe, P.M.

J. Lightwave Tech. LT-3(1): 154-158; Feb 85

The Bandwidth of a Multimode Fiber Chain;

Rodhe, P.

J. Lightwave Tech. LT-3(1): 145-154; Feb 85

Conference Report: Fiber Optics Emphasis on Single Mode;

Franzen, D.L.: Day, G.W.

1984 Optical Fiber Measurements Symp., in NBS JRES 90(1): 49 pp;

Jan-Feb 85

Attenuation of Multimode Fused Silica Optical Fibers Cooled to Liquid Helium Temperatures;

Engelsrath. A.: Larson, D.R.; Phelan, R.J., Jr.; Franzen, D.L.

Proc., SPIE, Vol 499: 124-130; Aug 84

Equivalent Step-Index Parameters in Single-Mode Fibers: Measurement and Applications;

Srivastava, R.

Proc., SPIE, Vol 500: 27-36; Aug 84

Measurement of Multimode Optical Fiber Attenuation: An NBS Special Test Service;

Gallawa, R.L.: Chamberlain, G.E.; Day, G.W.; Franzen, D.L.; Young, M. NBSIR 83-1691; Feb 84

Birefringence Measurements in Single Mode Optical Fiber;

Day, G.W.

Proc., SPIE, Vol 425: 72-79; 83 
Optical Fiber Metrology

Estimating Index Profiles of $1.3 \mu \mathrm{m}$ Single Mode Fibers by Near-Field Measurements at Blue Wavelengths;

Kim, E.M.; Franzen, D.L.; Young, M.; Rodhe, P.M.

J. Lightwave Tech. LT-1(4): 562-566; Dec 83

Optical Fiber Characterization Attenuation Frequency Domain Bandwidth and Radiation Patterns;

Chamberlain, G.E.; Day, G.W.; Franzen, D.L.; Gallawa, R.L.; Kim, E.M.; Young, M.

NBS SP 637, Vol II; Oct 83

Measurement of Multimode Optical Fiber Attenuation: An NBS Special Test Service;

Gallawa, R.L.; Chamberlain, G.E.; Day, G.W.; Franzen, D.L.; Young, M. NBS TN 1060; Jun 83

Optical Time-Domain Reflectometer Performance and Calibration Studies;

Danielson, B.L.

NBS TN 1064; Jun 83

Two-Dimensional Near-Field Contouring of Optical Fiber Cores;

Kim, E.M.; Franzen, D.L.

Proc., SPIE, Vol 355: 76-83; Jun 83

EIA Fiber Performance Measurement Standards;

Gallawa, R.L.; Franzen, D.L.

Photonics Spectra, 55-68; Apr 83

An Inter-Laboratory Measurement Comparison of Core Diameter on Graded-Index Optical Fibers;

Kim, E.M.; Franzen, D.L.

NBS SP 641; Oct 82

Measurement of the Core Diameter of Graded-Index Optical Fibers: An Interlaboratory Comparison;

Kim, E.M.; Franzen, D.L.

Appl. Opt. 21(19): 3443-3450; Oct 82

Optical Fiber Characterization;

Day, G.W.; Danielson, B.L.; Franzen, D.L.; Kim, E.; Young, M.

NBS SP 637, Vol I; Jul 82 
Calibration Reticle for Optical Fiber Near-Field Core Diameter Measurements; Kim, E.M.; Franzen, D.L.

Tech. Dig., Conf. on Prec. Electromagn. Meas.; May 82

Characterization of a Concentric-Core Fiber;

Danielson, B.L.; Franzen, D.L.; Gallawa, R.L.; Kim, E.M.; Young, M. NBSIR 82-1661; Apr 82

On the Definition of Fiber Numerical Aperture;

Gallawa, R.L.

Electro-Optical Systems Design, 47; Apr 82

Book Review: Principles of Optical Fiber Measurements by D. Marcuse;

Young, $\mathrm{M}$.

Laser Focus, 118-119; Jan 82

Optical Waveguide Communications Glossary;

Hanson, A.G.; Bloom, L.R.; Day, G.W.; Gallawa, R.L.; Gray, E.M.; Young, M. NBS HB 140; Jan 82

Long Optical Fiber Fabry Perot Interferometers;

Franzen, D.L.; Kim, E.M.

Appl. Opt. 20(23): 3991-3992; 81

An Optical Waveguide Communications Glossary Revised;

Hanson, A.G.; Bloom, L.R.; Day, G.W.; Young, M.; Gray, E.M.; Gallawa, R.L. NBS HB 140; Dec 81

Backscatter Signature Simulations;

Danielson, B.L.

NBS TN 1050; Dec 81

Book Review: Optical Fibre Communication (invited review);

Gallawa, R.

IEEE Spectrum 18(11): 81-82; Nov 81

Optical Fiber Index Profiles by Refracted-Ray Scanning;

Young, $\mathrm{M}$.

Appl. Opt. 20(19): 3415-3421; Oct 81 
Optical Fiber Metrology

Measurement of Optical Fiber Bandwidth in the Frequency Domain;

Day, G.W.

NBS TN 1046; Sep 81

Standardizing Test Conditions for Characterizing Fibers;

Franzen, D.L.; Day, G.W.; Gallawa, R.L.

Laser Focus 17: 103-105; Aug 81

Interlaboratory Measurement Comparison to Determine the Attenuation and Bandwidth of Graded-Index Fibers;

Franzen, D.L.; Day, G.W.; Danielson, B.L.; Chamberlain, G.E.; Kim, E.M.

Appl. Opt. 20(14): 2412-2419; Jul 15, 81

The Characterization of Optical Fiber Waveguides: A Bibliography with Abstracts 1970-1980;

Day, G.W.

NBS TN 1043; Jun 81

Refracted-Ray Scanning (Refracted Near-Field Scanning) for Measuring Index Profiles of Optical Fibers;

Young, $\mathrm{M}$.

NBS TN 1038; May 81

Present NBS Capability in Optical Fiber Measurements;

Day, G.W.; Franzen, D.L.

Proc., 1st Intl. DoD/Industry Fiber Optics Standards Conf., Washington, DC; Apr 81

Results of an Interlaboratory Measurement Comparison Among Fiber Manufacturers to Determine Attenuation Bandwidth and Numerical Aperture of Graded Index Optical Fibers;

Franzen, D.L.; Day, G.W.; Danielson, B.L.; Kim, E.M.

Dig., 3rd Intl. Conf. Integrated Optics and Optical Fiber Comm., San

Francisco, CA; Apr 81

Results of an Inter-Laboratory Measurement Comparison to Determine the Radiation Angle (NA) of Graded Index Optical Fibers;

Franzen, D.L.; Kim, E.M.

Appl. Opt. 20(7): 1218-1220; Apr 81

Backscatter Measurements in Optical Fibers;

Danielson, B.L.

NBS TN 1034; Feb 81 
Measurement of Far-Field and Near-Field Radiation Patterns from Optical Fibers;

Kim, E.M.; Franzen, D.L.

NBS TN 1032; Feb 81

Progress in Fiber Measurements;

Day, G.W.; Franzen, D.L.

Laser Focus, 52-56; Feb 81

The Role of Backscatter Signatures in Optical Fiber Characterization;

Danielson, B.L.

Tech. Dig., lst Intl. DoD Ind. Fiber Optics Congress, Apr 20, 1980, Washington, DC; 80

Linearity and Resolution of Refracted Near-Field Scanning Techniques;

Young, $\mathrm{M}$.

Tech. Dig., Symp. on Optical Fiber Measurements, in NBS SP 597; Oct 80

Calibration Technique for Refracted Near-Field Scanning of Optical Fibers;

Young, $M$.

Appl. Opt. 19: 2479-2480; Aug 80

An Assessment of the Backscatter Technique as a Means for Estimating Loss in Optical Waveguides;

Danielson, B.L.

NBS TN 1018; Feb 80

Measurement of Optical Fiber Bandwidth in the Time Domain;

Franzen, D.L.; Day, G.W.

NBS TN 1019; Feb 80

Measurement of Propagation Constants Related to Material Properties in High Bandwidth Optical Fibers;

Franzen, D.L.; Day, G.W.

IEEE J. Quant. Electron. QE-15(12); Dec 79

Limitations Imposed by Material Dispersion on the Measurement of Optical Fiber Bandwidth with Laser Diode Sources;

Franzen, D.L.; Day, G.W.

J. Opt. Soc. Am. 69(10): 1448; Oct 79 
Optical Fiber Metrology

Optical Waveguide Communications Glossary;

Hanson, A.G.; Bloom, L.R.; Day, G.W.; Gallawa, R.L.; Gray, E.M.; Young, M. NTIA SP 79-4; Sep 79

Time Domain Pulse Measurements and Computed Frequency Response of Optical Communications Components;

Andrews, J.R.; Young, M.

NBSIR 79-1620; Sep 79

Fiber Measurements: Quality and Cost;

Day, G.W.

Proc., Intl. Communications Conf., Boston, MA; Jun 79

Attenuation Measurements on Optical Fiber Waveguides: An Interlaboratory Comparison Among Manufacturers;

Day, G.W.; Chamberlain, G.E.

NBSIR 79-1608; May 79

A Simple First Positive System Nitrogen Laser for Use in Optical Fiber Measurements;

Franzen, D.L.; Danielson, B.L.; Day, G.W.

IEEE J. Quant. Electron. QE-14: 548; 78

Measurement Problems in Multimode Optical Waveguides;

Day, G.W.

Proc., Intl. Communications Conf. Vol 1, Jun 1978, Toronto, Canada; 78

Optical Fiber Phase Discriminator;

Danielson, B.L.

Appl. Opt. 17: 3665-3668; Nov 78

Fiber Optics Metrology at NBS;

Danielson, B.L.; Day, G.W.; Franzen, D.L.

Proc., Union Radio Scientifique Intl. Comm. Symp., Oct 3-7, 1977, Lannion, France, (Intl. Union of Radio Science, France), 430-431; Dec 77 


\section{INTEGRATED OPTIC METROLOGY}

Bending Induced Phase Shifts in Arbitrarily Bent Rectangular-Core Dual-Mode Waveguides;

Kumar, A.; Jindal, R.; Gallawa, R.L.

J. Lightwave Tech. 14(2): 196-201; Feb 96

Efficient Vector Solution for Optical Waveguides Using Galerkin's Method with HermiteGauss Basic Functions;

Weisshaar, A.; Li, J; Gallawa, R.L.

Proc., Conf. on Emerging Optoelectronic Technologies (DEOT-94), July 1822, 1994, Bandalore, India; 94

Mode Coupling and Loss on Tapered Optical Waveguides;

Gallawa, R.L.; Kumar, A.; Weisshaar, A.

Tech. Dig., Integ. Photonics Res. Topical Mtg., Feb 17-19, 1994, San

Francisco, CA, 3 ThD6-1-3, 57-59; 94

Mode Coupling and Loss on Tapered Optical Waveguides;

Gallawa, R.L.; Kumar, A.; Weisshaar, A.

Proc., Intl. Photonics Res. Conf., Feb 17-19, 1994, San Francisco, CA, ; 94

Bending-Induced Loss in Dual-Mode Rectangular Waveguides;

Kumar, A.; Gallawa, R.L.

Opt. Lett. 19(10): 707-709; May 15, 94

Bent Rectangular Core Waveguides: An Accurate Perturbation Approach;

Kumar, A.; Gallawa, R.L.

Microwave Opt. Tech. Lett. 7(6): 281-285; Apr 20, 94

Modal Characteristics of Bent Dual Mode Planar Optical Waveguides;

Kumar, A.; Gallawa, R.L.; Goyal, I.C.

J. Lightwave Tech. LT-12(4): 621-624; Apr 94

Symbolic Programming With Series Expansions: Applications to Optical Waveguides;

Gallawa, R.L.; Kumar, A.; Weisshaar, A.

Proc., 10th Annual Review of Progress in Applied Computational

Electromagnetics, Mar 21-26, 1994, Monterey, CA, 475-481; Mar 94

Guided-Wave Optics on Silicon: Physics, Technology, and Status;

Pal, B.P.

Progress in Optics, E. Wolf, ed., Elsevier Science Publishers B.Vol XXXII; 93 
Integrated Optic Metrology

Improved Variational Analysis of Inhomogeneous Optical Waveguides Using Airy Functions; Goyal, I.C.; Gallawa, R.L.; Ghatak, A.K.

J. Lightwave Tech. LT-11(10): 1575-1578; Oct 93

Bending-Induced Phase Shifts in Dual-Mode Planar Optical Waveguides;

Kumar, A.; Gallawa, R.L.

Opt. Lett. 18(17): 1415-1417; Sep 1, 93

Complex Propagation Constants for Nonuniform Optical Waveguides: Calculations;

Gallawa, R.L.

Microwave and Opt. Tech. Lett. 6(8): 490-493; Jun 20, 93

Accuracy of Eigenvalues: A Comparison of Two Methods;

Goyal, I.C.; Gallawa, R.L.; Ghatak, A.K.

J. Math. Phys. 34(3): 1169-1175; Mar 93

Modified Airy Function Method for the Analysis of Tunneling Problems in Optical

Waveguides and Quantum-Well Structures;

Roy, S.; Ghatak, A.K.; Goyal, I.C.; Gallawa, R.L.

IEEE J. Quant. Electron. QE-29(2): 340-345; Feb 93

Modal Properties of Circular and Noncircular Optical Waveguides;

Gallawa, R.L.; Goyal, I.C.; Ghatak, A.K.

Fiber Integ. Opt. 11: 25-50; 92

A New Method of Analyzing Planar Optical Waveguides;

Goyal, I.C.: Gallawa, R.L.; Ghatak, A.K.

Opt. Lett. 16: 30-32; 91

Low Coherence Optical Reflectometry of Laser Diode Waveguides;

Boisrober. C.Y.; Franzen, D.L.; Danielson, B.L.; Christensen, D.H.

Proc., SPIE, Vol 1474; 91

Optical Waveguide Analysis Using Modified Airy Functions;

Gallawa, R.L.; Goyal, I.C.; Ghatak, A.K.

Fiber Integ. Opt. 10: 1-10; 91

An Approximate Solution to the Scalar Wave Equation for Planar Optical Waveguides;

Goyal, I.C.; Gallawa, R.L.; Ghatak, A.K.

Appl. Opt. 30(21): 2985-2989; Jul 20, 91 
An Approximate Solution to the Wave Equation-Revisited;

Goyal, I.C.; Gallawa, R.L.; Ghatak, A.K.

J. Electromagn. Waves Appl. 5: 623-636; Jun 91

Bent Planar Waveguides and Whispering Gallery Modes: A New Method of Analysis;

Goyal, I.C.; Gallawa, R.L.; Ghatak, A.K.

J. Lightwave Tech. LT-8(5): 768-774; May 90

Analysis of Bends in Planar Optical Waveguides;

Gallawa, R.L.; Tu, Y.

Fiber Integ. Opt. 8: 87-97; 89

Optical Waveguide Attenuation Measured by Photothermal Displacement;

Hickernell, R.K.; Aust, J.A.; Larson, D.R.

Conf. Dig., 6th Intl. Topical Mtg. on Photoacoustic and Photothermal Phenomena, 310-311; Jul 89

Electrically Calibrated Photothermal Deflection Measurement for Separating Channel Waveguide Loss Mechanisms;

Hickernell, R.K.; Larson, D.R.; Phelan, R.J., Jr.

NBS SP 748, 65-68; Sep 88

Waveguide Loss Measurement Using Photothermal Deflection;

Hickernell, R.K.; Larson, D.R.; Phelan, R.J., Jr.; Larson, L.E.

Appl. Opt. 27(13): 2636-2638; Jul 1, 88 


\section{OPTICAL FIBER SENSORS}

Variation in Optical Disc Birefringence Measurements;

Rochford, K.B.; Kreger, S.T.; West, J.W.

Proc., SPIE, Vol 3864, Joint International Symposium on Optical Memory and Optical Data Storage 1999, Jul 11-15, 99, Koloa, Hawaii: 223-225; Jul 99

Optical Fiber Current Sensors in High Electric Field Environments;

Rose, A.H.; Etzel, S.M.; Rochford, K.B.

IEEE J. Lightwave Tech., 17(6): 1042-1048; Jun 99

Reconstruction of Minimum-Phase Group Delay from Fibre Bragg Grating

Transmittance/Reflectance Measurements;

Rochford, K.B.; Dyer, S.D.

Electron. Lett. 35(10): 838-839; May 13, 99

Demultiplexing of Interferometrically Interrogated Fiber Bragg Grating Sensors Using Hilbert Transform Processes;

Rochford, K.B.; Dyer, S.D.

IEEE J. Lightwave Tech., 17(5): 831-835; May 99; May 99

Dense Wavelength-Division Multiplexing of Interferometrically Interrogated Fiber Bragg Grating Sensors;

Rochford, K.B.; Dyer, S.D.

Proc., 13th Optical Fiber Sensors Conf., Apr 12-16, 1999, Kyongju, South Korea, W1-4 149-152; Apr 99

Electro-Optic Kerr Effects in Optical Fiber Current Sensors;

Rose, A.H.; Etzel, S.M.; Rochford, K.B.

Proc., 13th Optical Fiber Sensors Conf., Apr 12-16, 1999, Kyongju, South

Korea, TH1-1 354-357; Apr 99

High Frequency Response of Optical Magnetic Field Sensors Using Rare-Earth Iron Garnet Films;

Itoh, N.; Rochford, K.B.; Minemoto, H.; Ishizuka, S.

Proc., 13th Optical Fiber Sensors Conf., Apr 12-16, 1999, Kyongju, South Korea, TH1-5 370-373; Apr 99

Annealed Fiber: Properties and Applications;

Rose, A.H.; Espejo, R.J.; Craig, R.M.

Proc., IEEE Lasers and Electro-Optics Society, 11th Annual Mtg., Vol 1, Dec 1-4, 1998, Orlando, FL, 397-398; Dec 98 
The Observation of $\mathrm{OH}$ in Annealed Optical Fiber;

Rose, A.H.; Bruno, T.J.

J. Non-Crystalline Solids, 231, 280-285; Sep 98

Spectral Tailoring of an Erbium Superfluorescent Fiber Source;

Dyer, S.D.; Rochford, K.B.

Electron. Lett. 34(11): 1137-1139; May 28, 98

Interlaboratory Comparison of Polarization Crosstalk Measurement Methods in Terminated High-Birefringence Optical Fiber;

Craig, R.M.

Tech. Dig., Optical Fiber Communication Conf. (OFC'98), Feb 22-27, 1998, San Jose, CA, Vol 2: 180-181; Feb 98

Standards for Linear Retardance;

Rochford, K.B.; Rose, A.H.

Proc., SPIE, Vol 3121, Polarization: Measurement, Analysis, and Remote

Sensing, Jul 30-Aug 1, 97, San Diego, CA: 188-192; 97

Spectral Tailoring of an Erbium Superfluorescent Fiber Source;

Dyer, S.D.; Rochford, K.B.

Proc., 12th Intl. Conf. on Optical Fiber Sensors, Oct 27-31, 1997,

Williamsburg, VA, PD6-1 thru PD6-4; Oct 97

Accurate Interferometric Retardance Measurements;

Rochford, K.B.; Wang, C.M.

Appl. Opt. 36(25): 6473-6479; Sep 1, 97

Design and Performance of a Stable Linear Retarder;

Rochford, K.B.; Rose, A.H.; Williams, P.A.; Wang, C.M.; Clarke, I.G.; Hale, P.D.;

Day, G.W.

Appl. Opt. 36(25): 6458-6465; Sep 1, 97

Packaging Hygroscopic and Stress-Sensitive Optics for a Standard Retarder;

Rose, A.H.; Rochford, K.B.

Proc., SPIE, Vol 3132, Optomechanical Design and Precision Instruments, Jul 31-Aug 1, 1997, San Diego, CA: 120-126; Aug 97

Devitrification in Annealed Optical Fiber;

Rose, A.H.

J. Lightwave Tech. 15(5): 808-814; May 97 
Optical Fiber Sensors

NIST Study Investigates Retardance Uncertainty;

Rochford, K.B.; Rose, A.H.; Wang, C.-M Laser Focus World 33(5); 223-227; May 97

Verdet Constant Dispersion in Annealed Optical Fiber Current Sensors;

Rose, A.H.; Etzel, S.M.; Wang, C.-M.

J. Lightwave Tech. 15(5): 803-807; May 97

Simultaneous Laser Diode Emission and Detection for Fiber Optic Sensors;

Rochford, K.B.; Rose, A.H.

Optics and Photonics News 7(12); 35-36; Dec 96

Magneto-Optic Sensors Based on Iron Garnets;

Rochford, K.B.; Rose, A.H.; Day, G.W.

Proc., IEEE Lasers and Electro-Optics Society, 9th Annual Mtg, Vol1, Nov 1819, 1996, Boston, MA, 242-243; Nov 96

Twisting and Annealing Optical Fiber for Current Sensors;

Rose, A.H.; Ren, Z.B.; Day, G.W.

J. Lightwave Tech. 14(11): 2492-2498; Nov 96

Uncertainty in Null Polarimeter Measurements;

Rochford, K.B.; Wang, C.M.

NISTIR 5055; 15 pp; Oct 96

Magneto-Optic Sensors Based on Iron Garnets;

Rochford, K.B.; Rose, A.H.; Day, G.W.

IEEE Trans. Magn. 32(5): 4113-4117; Sep 96

Optical Retardance Standard: A Progress Report;

Rochford, K.B.; Rose, A.H.; Williams, P.A.; Clarke, I.; Hale, P.D.; Day, G.W. Proc., SPIE, Vol 2873, Japan Chapter's Intl. Symp. on Polarization Analysis and Applications to Device Technology, June 12-14, 1996, Yokohama, Japan: 208-209; Sep 96

Molecular Field Theory Analysis of Magneto-Optic Sensitivity of Gallium-Substituted Yttrium Iron Garnets;

Deeter, M.N.; Milian Bon, S.

Appl. Phys. Lett. 69(5): 702-704; Jul 29, 96 
Optical, Thermo-Optic, Electro-Optic, and Photoelastic Properties of Bismuth Germanate ( $\mathrm{Bi}_{4}$ $\mathrm{Ge}_{3} \mathrm{O}_{12}$ );

Williams, P.A.; Rose, A.H.; Lee, K.S.; Conrad, D.; Day, G.W.; Hale, P.D. Appl. Opt. 35(19): 3562-3569; Jul 1, 96

Fundamentals and Problems of Fiber Current Sensors;

Day, G.W.; Rochford, K.B.; Rose, A.H.

Proc., 11th Intl. Conf. on Optical Fiber Sensors, Advanced Sensing Photonics, May 21-24, 1996, Sapporo, Hokkaido, Japan; 124-129; May 96

Magneto-Optic Rotation Sensor Using a Laser Diode as Both Source and Detector;

Rochford, K.B.; Rose, A.H.

Proc., 11th Intl. Conf. on Optical Fiber Sensors, Advanced Sensing Photonics, May 21-24, 1996, Sapporo, Hokkaido, Japan; 686-689; May 96

Optical Retardance Standard: A Progress Report;

Rochford, K.B.; Rose, A.H.; Williams, P.A.; Clarke, I.; Hale, P.D.; Day, G.W. Proc., Workshop on Infrared and Millimeter Wave Polarimetry, SR RD-MG96-8, Dec 5-7, 1995, Redstone Arsenal, AL, 517-524; Apr 96

Fiber-Optic Faraday-Effect Magnetic-Field Sensor Based on Flux Concentrators;

Deeter, M.N.

Appl. Opt. 35(1): 154-157; Jan 1, 96

Self-Calibrating Optical Thermometer;

Rose, A.H.: Wyss, J.C.

Proc.. SPIE, Vol 2594, Self-Calibrating Optical Sensors and Systems Conf., 142-148: Jan 96

Simultaneous Laser-Diode Emission and Detection for Fiber-Optic Sensor Applications;

Rochford. K.B.; Rose, A.H.

Opt. Lett. 20(20): 1205-1207; Oct 15, 95

$\mathrm{X}$-ray Observation of Electroclinic Layer Constriction and Rearrangement in a Chiral Smetic- $A$ Liquid Crystal;

Rappapor. A.G.; Williams, P.A.; Thomas, B.N.; Clark, N.A.; Ros, M.B.; Walba, D.M.

Appl. Phys. Lett. 67(3): 362-364; Jul 17, 95 
Optical Fiber Sensors

Dielectric Spectroscopic Determination of Temperature Behavior of Electroclinic Parameters in the Liquid Crystal W317;

Williams, P.A.; Clark, N.A.

J. Appl. Phys. 78(1): 413-417; Jul 1, 95

High Sensitivity Fiber-Optic Magnetic Field Sensors Based on Iron Garnets;

Deeter, M.N.

IEEE Trans. Instrum. Meas. 44(2): 464-467; Apr 95

Domain Effects in Faraday Effect Sensors Based on Iron Garnets;

Deeter, M.N.

Appl. Opt. 34(4): 655-658; Feb 1, 95

Magnetooptic Effects;

Deeter, M.N.; Day, G.W.; Rose, A.H.

CRC Handbook of Laser Science and Technology, Supplement 2: Optical

Materials; Sec. 9.1, Crystals and Glasses, 5, 367-402; Jan 95

A Polarization Insensitive 3x3 Sagnac Current Sensor Using Polarizing Spun High-

Birefringence Fiber;

Clarke, I.; Rochford, K.B.; Rose, A.H.; Day, G.W.

Proc., SPIE, Vol 2360, 10th Optical Fibre Sensors Conf., Oct 11-13, 1994, Glasgow, Scotland: Post-Deadline; 94

An Improved Annealing Technique for Optical Fiber;

Rose, A.H.; Ren, Z.B.; Day, G.W.

Proc., SPIE, Vol 2360, 10th Optical Fibre Sensors Conf., Oct 11-13, 1994, Glasgow, Scotland: 306-309; 94

Effect of Semiconductor Laser Characteristics on Optical Fiber Sensor Performance;

Rochford, K.B.; Rose, A.H.; Clarke, I.; Day, G.W.

Proc., SPIE, Vol 2148, Laser Diode Technology and Application VI, Jan 25-

27, 1994, Los Angeles, CA: 269-279; 94

Faraday Effect Sensors for Magnetic Field and Electric Current;

Day, G.W.; Deeter, M.N.; Rose, A.H.; Rochford, K.B.

Proc., SPIE, Vol 2292, Fiber Optic and Laser Sensors XII, Jul 25-27, 1994, San Diego, CA: 42-47; 94 
Faraday Effect Sensors for Magnetic Field and Electric Current;

Day, G.W.; Deeter, M.N.; Rose, A.H.; Rochford, K.B.

Proc., SPIE, Vol 2341, Interferometric Fiber Sensing, May 17-20, 1994, Warsaw, Poland: 90-95; 94

Faraday Effect Current Sensor with Improved Sensitivity-Bandwidth Product;

Rochford, K.B.; Rose, A.H.; Deeter, M.N.; Day, G.W.

Proc., SPIE, Vol 2360, 10th Optical Fibre Sensors Conf., Oct 11-13, 1994, Glasgow, Scotland: $32-35$; 94

Optical Wheel-Rotation Sensor;

Veeser, L.R.; Rodriguez, P.; Forman, P.R.; Deeter, M.N.

Proc., SPIE, Vol 2360, 10th Optical Fibre Sensors Conf., Oct 11-13, 1994, Glasgow, Scotland: 388-391; 94

Standard Polarization Components: Progress Toward an Optical Retardance Standard;

Rochford, K.B.; Rose, A.H.; Williams, P.A.; Clarke, I.; Day, G.W.

Proc., SPIE, Vol 2265, Polarization Analysis and Measurement II, Jul 25-27, 1994, San Diego, CA: 2-8; 94

Faraday Effect Current Sensor with Improved Sensitivity-Bandwidth Product;

Rochford, K.B.; Rose, A.H.; Deeter, M.N.; Day, G.W.

Opt. Lett. 19(22): 1903-1905; Nov 15, 94

Novel Bulk Iron Garnets for Magneto-Optic Magnetic Field Sensing;

Deeter, M.N.; Milian Bon, S.; Day, G.W.

Proc., IEEE Trans. Magn., 6th Joint Magnetism and Magnetic Materials Intl. Mag. Conf., Albuquerque, NM, Jun 20-23, 1994, 4464-4466; Nov 94

Polarization Dependence of Response Functions in 3x3 Sagnac Optical Fiber Current Sensors; Rochford, K.B.; Day, G.W.; Forman, P.R.

J. Lightwave Tech. LT-12(8): 1504-1509; Aug 94

Optical Fiber Sensors: Accelerating Applications in Navy Ships;

Day, G.W.; Lovely, P.S.; Whitesel, H.K.; Hickernell, R.K.

NISTIR 5018, 58 pp; May 94

Faraday Effect Sensors for Magnetic Field and Electric Current;

Rochford, K.B.; Day, G.W.; Deeter, M.N.; Rose, A.H.

Proc., DoD Fiber Optics '94, Optical Networks in the Concept of a Global

Grid, Mar 22-24, 1994, McLean, VA, 21-24; Apr 94 
Optical Fiber Sensors

Interlaboratory Comparison of Polarization-Holding Parameter Measurements on High Birefringence Optical Fiber;

Craig, R.M.; Tang, D.; Day, G.W.

Tech. Dig., 2nd Optical Fibre Meas. Conf., Sep 21-22, 1993, Torino, Italy, $177-180 ; 93$

Self Calibrating Fiber Optic Sensors: Potential Design Methods;

Whitesel, H.K.; Miller, C.A.; Day, G.W.; Rose, A.H.

Proc., Intl. Symp. on Spectral Sensing Research (ISSSR), Vol I, Nov 15-20, 1992, Maui, HI, 324-341; 93

Studies of the Higher Order Smectic Phase of the Large Electroclinic Effect Material W317;

Williams, P.A.; Komitov, L.; Rappaport, A.G.; Thomas, B.N.; Clark, N.A.; Walba, D.M.; Day, G.W.

Liquid Crystals 14(4): 1095-1105; 93

Wideband Current and Magnetic Field Sensors Based on Iron Garnets;

Deeter, M.N.; Rochford, K.B.; Rose, A.H.; Day, G.W.

Tech. Program, 3rd Annual DARPA Symp. on Photonic Systems for Antenna Applications, Jan 20-22, 1993, Monterey, CA; 93

Magneto-Optic Magnetic Field Sensors Based on Uniaxial Iron Garnet Films in Optical Waveguide Geometry;

Deeter, M.N.; Day, G.W.; Wolfe, R.; Fratello, V.J.

IEEE Trans. Magn. TM- 29(6): 3402-3404; Nov 93

Submicroampere-per-root-hertz Current Sensor Based on the Faraday Effect in Ga:YIG;

Rose, A.H.; Deeter, M.N.; Day, G.W.

Opt. Lett. 18(17): 1471-1473; Sep 1, 93

Magneto-Optic Magnetic Field Sensor with $1.4 \mathrm{pT} / \sqrt{ } \mathrm{Hz}$ Minimum Detectable Field at $1 \mathrm{kHz}$;

Deeter, M.N.; Day, G.W.; Beahn, T.J.; Manheimer, M.

Electron. Lett. 29(11): 993-994; May 27, 93

Polarization Dependence of Response Functions in 3×3 Sagnac Optical Fiber Current Sensors;

Rochford, K.B.; Day, G.W.

Proc., 9th Optical Fiber Sensors Conf., May 4-6, 1993, Firenze, Italia, Post-

Deadline; May 6, 93 
High-Speed High Sensitivity Magnetic Field Sensors Based on the Faraday Effect in Iron Garnets;

Deeter, M.N.

Proc., 9th Optical Fiber Sensors Conf., May 4-6, 1993, Firenze, Italia, 409414; May 93

Self-Calibrating Fiber Optic Sensors: Potential Design Methods;

Whitesel, H.K.; Day, G.W.; Rose, A.H.; Miller C.A.

Tech. Report, Naval Surface Warfare Center, CARDIVNSWC-TR-80-92/15;

May 93

Magneto-Optic Magnetic Field Sensors Based on Uniaxial Iron Garnet Films in Optical Waveguide Geometry;

Deeter, M.N.; Day, G.W.; Wolfe, R.; Fratello, V.J.

Dig., Inter. Mag. Conf., Apr 13-16, 1993, Stockholm, Sweden, EF-09; Apr 93

Magneto-Optic Characterization of Iron Garnet Crystals Using Photoelastic Modulation;

Deeter, M.N.; Williams, P.A.

Dig., Inter. Magn. Conf., Apr 13-16, 1993, Stockholm, Sweden, JQ-02; Apr 93

Domain Effects in Faraday Effect Sensors Based on Iron Garnets;

Deeter, M.N.

Proc., 8th Optical Fibers Sensors Conf., Jan 29-31, 1992, Monterey, CA, Post Deadline paper; 92

Faraday Effect Current Sensors;

Day, G.W.; Deeter, M.N.; Rose, A.H.

Proc., 17th Australian Conf. on Optical Fibre Tech., Nov 30-Dec 3, 1992,

Hobart, Tasmania, Australia, 20-27; 92

Magneto-Optic Characterization of Iron Garnet Crystals Using Photoelastic Modulation;

Deeter, M.N.; Williams, P.A.

IEEE Trans. Magn. MAG-28: 3234-3236;' 92

Magneto-Optic Characterization of Iron Garnet Crystals Using Photoelastic Modulation;

Deeter, M.N.; Williams, P.A.

Tech. Dig., Intl. Mag. Conf., Apr 13-16, 1992, St. Louis, MO, JQ-02; 92 
Optical Fiber Sensors

Submicroampere per Root Hz High Bandwidth Current Sensor Based on the Faraday Effect in Ga:YIG;

Rose, A.H.; Deeter, M.N.; Day, G.W.

Proc., 8th Optical Fibers Sensors Conf., Jan 29-31, 1992, Monterey, CA, 394$397 ; 92$

High Frequency Magnetic Field Sensors Based on the Faraday Effect in Garnet Thick Films;

Wolfe, R.; Gyorgy, E.M.; Lieberman, R.A.; Fratello, V.J.; Licht, S.J.; Deeter, M.N.; Day, G.W.

Proc., Optical Fibers Sensors Conf., Jan 29-31, 1992, Monterey, CA, 390-393, 92; and Appl. Phys. Lett. 60(17): 2048-2050; Apr 27, 92

High Sensitivity High Speed Current Sensor Based on the Faraday Effect in Ga:YIG;

Rose, A.H.; Deeter, M.N.; Day, G.W.

Proc., 8th Optical Fiber Sensors Conf., 394-397; Jan 92

Faraday Effect Sensors: A Review of Recent Progress;

Day, G.W.; Deeter, M.N.; Rose, A.H.

Proc., Intl. Optical Fiber Sensors Conf., Oct. 9-11, 1991, Wuhan, China, 1126; 91

Faraday-Effect Magnetic Field Sensors Based on Substituted Iron Garnets;

Deeter, M.N.; Rose, A.H.; Day, G.W.

Proc., SPIE, Fiber Optics and Laser Sensors Conf., Sep 16-21, 1990, Vol 1367: 243-248; 91

Fiber-Optic Sensors for Aerospace Electrical Measurements: An Update;

Patterson, R.L.; Rose, A.H.; Tang, D.; Day, G.W.

NASA Tech. Memorandum 104454, 1-4; 91

Fiber-Optic Sensors for Aerospace Electrical Measurements: An Update;

Rose, A.H.; Tang, D.; Day, G.W.

Proc., 26th IECEC-91, Aug 4-9, 1991, Boston, MA, 180-183; 91

Large Electroclinic Effect in New Liquid Crystal Material;

Williams, P.A.; Ros, M.B.; Clark, N.A.; Vohra, R.T.; Walba, D.M.; Wand, M.D. Proc., 3rd Intl. Conf. on Ferroelectric Liquid Crystals; Ferroelectrics, 121(14), 143-146; 91

Sensitivity Limits to Ferrimagnetic Faraday Effect Magnetic Field Sensors;

Deeter, M.N.; Rose, A.H.; Day, G.W.; Samuelson, S.

J. Appl. Phys. 70(10): 6407-6409; Nov 15, 91 
Optical Fiber Voltage Sensors for Broad Temperature Ranges;

Rose, A.H.; Day, G.W.

Proc., SPIE, Fiber Optic Components and Reliability, Vol 1580: 95-103;

Sep 91

Annealing of Linear Birefringence in Single-Mode Fiber Coils: Application to Optical Fiber Current Sensors;

Tang, D.; Rose, A.H.; Day, G.W.; Etzel, S.M.

J. Lightwave Tech. LT-9(8): 103-1037; Aug 91

Compensation for the Temperature Dependence of the Faraday Effect in Diamagnetic Materials: Application to Optical Fiber Sensors;

Williams, P.A.; Day, G.W.; Rose, A.H.

Electron. Lett. 27(13): 1131-1132; Jun 20, 91

Performance and Limitations of Faraday Effect Sensors;

Rose, A.H.; Deeter, M.N.; Tang, D.; Day, G.W.

Proc., 178th Mtg. of the Electrochem. Soc. Extended Abstracts; Jun 91

Temperature Dependence of the Verdet Constant in Several Diamagnetic Glasses;

Williams, P.A.; Rose, A.H.; Day, G.W.; Deeter, M.N.; Milner, T.E.

Appl. Opt. Lett. 30: 1176-1178; Apr 91

A Fiber-Optic Current Sensor for Aerospace Applications;

Patterson. R.L.; Rose, A.H.; Tang, D.; Day, G.W.

Proc., 25th Intersociety Energy Conversion Eng. Conf., Aug 12-17, 1990,

Reno. NV, NASA Tech. Memorandum 103152, 500-504; 90

Faraday Effect Current Sensing Using a Sagnac Interferometer with a 3x3 Coupler;

Veeser. L.R.: Day, G.W.

Proc.. 7th Optical Fibre Sensors Conf., Dec 3-6, 1990, Sydney, Australia, 325-328: 90

High Speed Magnetic Field Sensors Based on Iron Garnets;

Deeter, M.N.: Rose, A.H.; Day, G.W.

Proc.. DoD Fiber Optics Conf. '90, Mar 20-23, 1990, McLean, VA, 423-424; 90

Iron-Garnet Magnetic Field Sensors with 100 pT/ $/ \mathrm{Hz}$ Noise-Equivalent Field;

Deeter, M.N.; Rose, A.H.; Day, G.W.

Proc., 7th Optical Fibre Sensors Conf., Dec 3-6, 1990, Sydney, Australia, 341-344; 90 
Optical Fiber Sensors

Optical Fiber Current Sensors with Temperature Stabilities near the Material Limit; Tang, D.; Rose, A.H.; Day, G.W.

Tech. Dig., 7th Intl. Optical Fiber Sensors Conf., Dec 2-7, 1990, Sydney, Australia; 90

Practical Considerations in the Design of Optical Fiber Current Sensors;

Tang, D.; Rose, A.H.; Day, G.W.

Proc., Intl. Congress on Optical Science and Engineering, Mar 12-15, 1990, The Hague, The Netherlands; 90

Fast Sensitive Magnetic Field Sensors Based on the Faraday Effect in YIG;

Deeter, M.N.; Rose, A.H.; Day, G.W.

J. Lightwave Tech. LT-8(12): 1838-1842; Dec 90

Performance and Limitations of Faraday Effect Sensors;

Rose A.; Deeter, M.N.; Tang, D.; Day, G.W.

J. Electrochem. Soc., Extended Abstracts of 178th Mtg., Vol 90-2: 1093;

Oct 90

Polarimetric Magnetic Field Sensors Based on Yttrium Iron Garnet;

Deeter, M.N.; Rose, A.H.; Day, G.W.

Tech. Dig., Lasers and Electro-Optics Society Mtg., (LEOS '89) Oct 17-20, 1989, Orlando, FL, M7 3, 110; 89

Recent Advances in Faraday Effect Sensors;

Day, G.W.

Proc., Intl. Optical Fiber Sensors Conf., Sep 1989, Paris, France, 250-254; 89

New Compensation Method for Bulk Optical Sensors with Multiple Birefringences;

Lee, K.S.

Appl. Opt. 28(11): 2001-2011; Jun 89

Faraday Effect Sensors: The State of the Art;

Day, G.W.; Rose, A.H.

Proc., SPIE, Vol 985: 138-150; 88

Optical Fiber Sensors for Electromagnetic Quantities;

Day, G.W.; Lee, K.S.; Rose, A.H.; Veeser, L.R.; Papatheofanis, B.; Whitesel, H.K.

Proc., DoD Fiber Optics Conf. and ISA Annual Conf., 205-207; 88 
Stability of Birefringent Linear Retarders (Waveplates);

Hale, P.D.; Day, G.W.

Appl. Opt. 27: 5146-5151; Dec 15, 88

Effect of Multiple Internal Reflections on the Stability of Electrooptic and Magnetooptic Sensors;

Lee, K.S.; Day, G.W.

Appl. Opt. 27: 4609-4611; Nov 15, 88

Progress in the Development of Miniature Optical Fiber Current Sensors;

Tang, D.; Day, G.W.

Proc., Lasers and Electro-optics Society Annual Mtg., 306-307; Nov 88

Optical Fiber Sensors for the Measurement of Electromagnetic Quantities;

Rose, A.H.; Day, G.W.; Lee, K.S.; Tang, D.; Veeser, L.R.; Papatheofanis, B.; Whitesel, H.K.

Proc., Sensors Expo 1988, 209A-1-3; Sep 88

Fiber Optic Faraday Rotation Current Sensor;

Veeser, L.R.; Day, G.W.

Proc., 4th Intl. Megagauss Field Generation and Related Topics Conf.; 87

Limits to the Precision of Electro-optic and Magneto-optic Sensors;

Day, G.W.; Hale, P.D.; Deeter, M.N.; Milner, T.E.; Conrad, D.; Etzel, S.M.

Electric Power Research Institute (EPRI) EL-5431 1; Mar 87

Limits to the Precision of Electro-Optic and Magneto-Optic Sensors;

Day, G.W.; Hale, P.D.; Deeter, M.; Milner, T.E.; Conrad, D.; Etzel, S.M.

NBS TN 1307, 128 pp; Mar 87

Fiber Optic Sensing of Pulsed Currents;

Veeser, L.; Chandler, G.I.; Day, G.W.

Proc., SPIE, Vol 648: 197-212; 86

Progress in the Design of Optical Fiber Sensors for the Measurement of Pulsed Electric Currents;

Day, G.W.; Veeser, L.R.; Chandler, G.I.; Cernosek, R.W.

Proc., Workshop on Measurements of Elect. Quan. in Pulsed Power Systems, Mar. 1986, Gaithersburg, MD, 58-63; 86 
Optical Fiber Sensors

Compact Fiber Sensors for the Measurement of Low Level Electric Currents;

Day, G.W.

Proc., 4th Intl. Optical Fiber Sensors Conf., Tokyo, Japan, 81-84; Oct 86

Annealing of Bend-Induced Birefringence in Fiber Current Sensors;

Day, G.W.; Etzel, S.M.

Tech. Dig., 5th Intl. Conf. on Integ. Opt. and Opt. Fibre Communication/11th European Conf. on Opt. Communication, IOOC-ECOC, Vol 1, Oct 1-4, 1985, Venice, Italy, 871-874; 85

Optical Fiber Sensors for the Measurement of Pulsed Electric Currents;

Day, G.W.; McFadden, J.D.O.; Veeser, L.R.; Chandler, G.I.; Cernosek, R.W. Proc., NATO/AGARD (Advisory Group for Aerospace Research and Development) Guided Optical Structures in the Military Environment, Sep 23-27, 1985, Istanbul, Turkey, AGARD-CPP-383: 8-1 to 8-9; 85 


\section{FIBER AND DISCRETE COMPONENTS}

Accurate Wavelength Calibration References for Wavelength Division Multiplexing;

Gilbert, S.L.; Swann, W.C.

Tech. Dig., Optical Fiber Communication Conf. (OFC'99), Feb 21-26, 1999,

San Diego, CA, Conf. Edition, THS2-1/267-269; Feb 99

Hydrogen Cyanide $\mathrm{H}^{13} \mathrm{C}^{14} \mathrm{~N}$ Absorption Reference for $1530 \mathrm{~nm}$ to $1560 \mathrm{~nm}$ Wavelength

Calibration-SRM 2519;

Gilbert, S.L.; Swann, W.C.; Wang, C-M

NISTSP 260-13, NIST Standard Reference Material 2519, 20 pp; Nov 98

Accurate Polarization Dependent Loss Measurement and Calibration Standard Development;

Craig, R.M.; Gilbert, S.L.; Hale, P.D.

Tech. Dig., Symp. on Optical Fiber Meas., in NIST SP 930, 5-8; Sep 98

High-Resolution, Nonmechanical Approach to Polarization-Dependent Transmission Measurements;

Craig, R.M.; Gilbert, S.L.; Hale, P.D.

J. Lightwave Tech. 16(7): 1285-1294; Jul 98

Acetylene ${ }^{12} \mathrm{C}_{2} \mathrm{H}_{2}$ Absorption Reference for $1510-1540 \mathrm{~nm}$ Wavelength Calibration-SRM 2517;

Gilbert, S.L.; Swann, W.C.

NIST SP 260-133, NIST Standard Reference Material 2517, 34 pp; Jan 98

Stability of Short, Single-Mode Erbium-Doped Fiber Lasers;

Svalgaard, M.; Gilbert, S.L.

Appl. Opt. 36(21): 4999-5005; Jul 20, 97

Laser Cooling;

Gilbert, S.L.; Wieman, C.E.

Macmillan Encyclopedia of Physics 2; J. Rigden, ed. (Simon and Schuster Macmillan, New York, 1996): 836-838; Nov 96

Electronically Tunable Fiber Laser for Optical Pumping of $3 \mathrm{He}$ and $4 \mathrm{He}$;

Stephens, E.F.; Patrick, H.; Gilbert, S.L.

Rev. Sci. Instrum. 67(3): 843-844; Mar 96

Decay of Bragg Gratings in Hydrogen-Loaded Optical Fibers;

Patrick, H.; Gilbert, S.L.; Lidgard, A.

Tech. Dig., Optical Fiber Communication Conf. (OFC'95), Feb 26-Mar 3, 1995, San Diego, CA, Vol 8: 179-180; 95 
Fiber and Discrete Components

Annealing of Bragg Gratings in Hydrogen-Loaded Optical Fiber;

Patrick, H.; Gilbert, S.L.; Lidgard, A.; Gallagher, M.D. J. Appl. Phys. 78(5): 2940-2945; Sep 1, 95

Comparison of UV Photosensitivity and Fluorescence During Fiber Grating Formation;

Patrick, H.; Gilbert, S.L.

Tech. Dig., Photosensitivity and Quadratic Nonlinearity in Glass Waveguides, Fundamentals and Applications, Vol 22, Sep 9-11, 1995, Portland, OR, (Optical Society of America, Washington, DC, 1995): 148-151; Sep 1, 95

Inexpensive Laser Cooling and Trapping Experiment for Undergraduate Laboratories;

Wieman, C.E.; Flowers, G.E.; Gilbert, S.L.

Am. J. Phys. 63(4): 317-330; Apr 95

Growth Characteristics of Fiber Gratings;

Gilbert, S.L.; Patrick, H.

Proc., Conf. on Lasers and Electro-Optics, Oct 31-Nov 3, 1994, Boston, MA, 131-132; Nov 94

Decrease of Fluorescence in Optical Fiber During Exposure to Pulsed or Continuous-Wave Ultraviolet Light;

Patrick, H.; Gilbert, S.L.; Lidgard, A.

Opt. Mater. 3(94): 209-216; Aug 94

Comparison of UV-Induced Fluorescence and Bragg Grating Growth in Optical Fiber;

Gilbert, S.L.; Patrick, H.

Tech. Dig., Conf. on Lasers and Electro-Optics (CLEO '94), Vol 8, May 8-13, 1994, Anaheim, CA, 244; May 94

Bragg Gratings in Optical Fibers Produced by a Continuous-Wave Ultraviolet Source;

Patrick, H.; Gilbert, S.L.

Proc., Conf. on Lasers and Electro-Optics, May 2-7, 1993, Baltimore, MD, 508; 93

Frequency Stabilization of a Fiber Laser to Rubidium: A High-Accuracy $1.53 \mu \mathrm{m}$ Wavelength Standard;

Gilbert, S.L.

Proc., SPIE, Vol 1837: 146-153; 93 
High-Resolution Spectroscopy of Laser Cooled Rubidium in a Vapor-Cell Trap;

Gilbert, S.L.

Proc., Quant. Electron. and Science Conf., May 2-7, 1993, Baltimore, MD, $212 ; 93$

Optical Probing of Cold Trapped Atoms;

Fox R.W.; Gilbert, S.L.; Hollberg, L.; Marquardt, J.H.; Robinson, H.G.

Opt. Lett. 18: 1456-1458; 93

Growth of Bragg Gratings Produced by Continuous-Wave Ultraviolet Light in Optical Fiber;

Patrick, H.; Gilbert, S.L.

Opt. Lett. 18(18): 1484-1486; Sep 15, 93

Laser Cooling and Trapping for the Masses;

Gilbert, S.L.; Wieman, C.E.

Opt. and Photonics News 4: 8-14; Jul 93

Atomic Physics Tests of Nonlinear Quantum Mechanics;

Bollinger, J.J.; Heinzen, D.J.; Itano, W.M.; Gilbert, S.L.; Wineland, D.J.

Proc., Santa Fe Workshop, Foundations of Quantum Mechanics, World

Scientific, Singapore, 40; 92

Moderate-Accuracy Wavelength Standards for Optical Communications;

Gilbert, S.L.; Drapela, T.J.; Franzen, D.L.

Tech. Dig., Optical Fiber Meas. Symp., in NIST SP 839, 191-194; Sep 92

A $303 \mathrm{MHz}$ Frequency Standard Based on Trapped $\mathrm{Be}^{+}$Ions;

Bollinger, J.J.; Heinzen, D.J.; Itano, W.M.; Gilbert, S.L.; Wineland, D.J.

IEEE Trans. Instrum. Meas. IM-40: 126-128; 91

Atomic Physics Tests of Nonlinear Quantum Mechanics;

Bollinger, J.J.; Heinzen, D.J.; Itano, W.M.; Gilbert, S.L.; Wineland, D.J.

Proc., 12th Intl. Conf. on Atomic Physics, 461; 91

Frequency Stabilization of a Tunable Erbium-Doped Fiber Laser;

Gilbert, S.L.

Opt. Lett. 16: 150-152; 91

Dual Wavelength Pulse Generation Using a Mode-Locked Erbium-Doped Fiber Ring Laser;

Schlager, J.B.; Kawanishi, S.; Saruwatari, M.

Electron. Lett. 27(22): 2072-2073; Oct 91 
Fiber and Discrete Components

High Resolution Spectroscopy Using Fiber Lasers;

Gilbert, S.L.

Proc., 10th Intl. Conf. on Laser Spectroscopy, Jun 17-22, 1991, Font-Romeu, France, 359-364; Jun 91

Wavelength Measurement System for Optical Fiber Communications;

Obarski, G.E.

NIST TN 1336, 44 pp; Feb 90 


\section{DIELECTRIC MATERIALS AND DEVICES}

Erbium and Ytterbium Waveguide Lasers in Phosphate Glass;

Funk, D.S.; Peters, P.M.; Veasey, D.L.; Sanford, N.A.; Hayden, J.S.

OSA TOPS on Advanced Solid-State Lasers, 1999 26: 157-159; 99

Ion-Exchanged $\mathrm{Er}^{3+} / \mathrm{Yb}^{3+}$ Glass Waveguide Lasers in Silicate Glasses;

Peters, P.M.; Veasey, D.L.; Funk, D.S.; Sanford, N.A.; Houde-Walter, S.N.; Hayden, J.S.

OSA TOPS on Advanced Solid-State Lasers, 1999 26: 160-162; 99

Laser Arrays May Provide DWDM Measurement Tool;

Veasey, D.L.; Funk, D.S.; Sanford, N.A.; Hayden, J.S.; Bendett, M.

Laser Focus World 35(5): 203-210; May 99

Arrays of Distributed-Bragg-Reflector Waveguide Lasers at $1536 \mathrm{~nm}$ in $\mathrm{Yb} / \mathrm{Er}$ Codoped Phosphate Glass;

Veasey, D.L.; Funk, D.S.; Sanford, N.A.; Hayden, J.S.

Appl. Phys. Lett. 74(6): 789-791; Feb 8, 99

Erbium/Ytterbium-Codoped Glass Waveguide Laser Producing $170 \mathrm{~mW}$ or Output Power at $1540 \mathrm{~nm}$;

Funk, D.S.; Veasey, D.L.; Peters, P.M.; Sanford, N.A.; Fontaine, N.H.; Hayden, J.S.

Tech. Dig., Optical Fiber Communication Conf. (OFC'99/IOOC), Feb 23, 1999, San Diego, CA, Conf. Edition, TuC5-1/32-34; Feb 99

Nonlinear Optical Characterization of $\mathrm{LiNbO}_{3}$. I. Theoretical Analysis of Maker Fringe Patterns for X-Cut Wafers;

Sanford, N.A.; Aust, J.A.

J. Opt. Soc. Am. B 15(12): 2885-2909; Dec 98

Dual Wavelength, 980 nm-Pumped, Er/Yb-Codoped Waveguide Laser in Ti:LiNbO ${ }_{3}$;

Amin, J.; Aust, J.A.; Veasey, D.L.; Sanford, N.A.

Electron. Lett. 34(5): 456-458; Mar 5, 98

Maker Fringe Mapping of $\mathrm{LiNbO}_{3}$ Wafers;

Sanford, N.A.; Aust, J.A.

OSA TOPS on Lasers and Optics for Manufacturing, 1996 9: 23-32; 97 
Dielectric Materials and Devices

Maker Fringe Analysis of Z-Cut Lithium Niobate;

Aust, J.A.; Sanford, N.A.; Amin, J.

Proc., IEEE Lasers and Electro-Optics Society, 10th Annual Mtg., Vol 1, Nov 10-13, 1997, San Francisco, CA, 114-115; Nov 97

Time-Dependent Modeling of Erbium-Doped Waveguide Lasers in Lithium Niobate Pumped at 980 and $1480 \mathrm{~nm}$;

Veasey, D.L.; Gary, J.M.; Amin, J.; Aust, J.A.

IEEE J. Quantum Elect. 33(10): 1647-1662; Oct 97

Imaging of Domain-Inverted Gratings in $\mathrm{LiNbO}_{3}$ by Electrostatic Force Microscopy;

Bluhm, H.; Wadas, A.; Wiesendanger, R.; Roshko, A.; Aust, J.A.; Nam, D. Appl. Phys. Lett. 71(1): 146-148; Jul 7, 97

Growth Morphologies of Heteroepitaxial Rutile Films on Sapphire Substrates;

Morris-Hotsenpiller, P.A.; Roshko, A.; Lowekamp, J.B.; Rohrer, G.S.

J. Crystal Growth 174: 424-433; Jul 97

$980 \mathrm{~nm}$-Pumped Er- and $\mathrm{Er} / \mathrm{Yb}$-Doped Waveguide Lasers in $\mathrm{LiNbO}_{3}$;

Amin, J.; Aust, J.A.; Veasey, D.L.;

Proc., ECIO '97 8th European Conference on Integrated Optics and Technical

Exhibition, April 2-4, 1997, Stockholm, Sweden, PD8-1 - PD8-4; Jun 97

Examination of Domain-Reversed Layers in Z-Cut $\mathrm{LiNbO}_{3}$ Using Maker Fringe Analysis, Atomic Force Microscopy, and High-Resolution X-Ray Diffraction Imaging;

Aust, J.A.; Steiner, B.; Sanford, N.A.; Fogarty, G.; Yang, B.; Roshko, A.; Amin, J.; Evans, C.

Tech. Dig., Conf. on Lasers and Electro-Optics (CLEO '97), Vol 11, May 1823, 1997, Baltimore, MD, 485-486; May 97

Rigorous Scalar Modeling of Er and Yb/Er-Doped Waveguide Lasers;

Veasey, D.L.; Gary, J.M.; Amin, J.

Proc., SPIE, Vol 2996, Rare-Earth-Doped Devices, Feb 8-14, 1997, San Jose, CA: 109-120; May 97

Stable CW Operating Waveguide Lasers at Room-Temperature in Rare-Earth-Diffused Lithium Niobate;

Amin, J.; Aust, J.A.; Sanford, N.A.

Proc., SPIE, Vol 2996, Rare-Earth-Doped Devices, Feb 8-14, 1997, San Jose, CA: 166-171; May 97 
Waveguide Lasers by Ion-Exchange in Er-Doped Glass;

Amin, J.; Veasey, D.L.; Sanford, N.A.; Hayden, J.S.

Proc., SPIE, Vol 2996, Rare-Earth-Doped Devices, Feb 8-14, 1997, San Jose, CA: 135-142; May 97

Z-Propagating Waveguide Lasers in Rare-Earth-Doped Ti: $\mathrm{LiNbO}_{3}$;

Amin, J.; Aust, J.A.; Sanford, N.A.

Appl. Phys. Lett. 69(25): 3785-3787; Dec 16, 96

Integrated Optical Polarization-Discriminating Receiver in Glass;

Veasey, D.L.; Larson, D.R.

J. Lightwave Tech. 13(11): 2244-2249; Nov 95

Distributed Feedback Lasers in Rare-Earth-Doped Phosphate Glass;

Veasey, D.L.; Malone, K.J.; Aust, J.A.; Sanford, N.A.; Roshko, A.

Proc., 7th European Conf. on Integrated Optics, Apr 3-6, 1995, Delft, The Netherlands, 579-581; Apr 6, 95

Glasses for Waveguide Lasers;

Malone, K.J.; Veasey, D.L.; Sanford, N.A.; Hayden, J.S.

Proc., SPIE, Vol 2287, Properties and Characteristics of Glass III, Jul 28-29, 1994, San Diego, CA: 75-87; 94

Integrated-Optical Devices in Rare-Earth-Doped Glass;

Malone, K.J.

Proc., SPIE, Vol CR53, Glass Integrated Optics and Optical Devices, Jul 2425, 1994, San Diego, CA: 132-156; 94

Using Secondary Ion Mass Spectrometry (SIMS) to Characterize Optical Waveguide Materials;

Novak, S.W.; Zavada, J.M.; Malone, K.J.

Proc., 28th Annual Microbeam Analysis Society Mtg., Jul 31-Aug 5, 1994, New Orleans, LA, 167-168; 94

Passively Q-Switched Nd-Doped Waveguide Laser;

Aust, J.A.; Malone, K.J.; Veasey, D.L.; Sanford, N.A.; Roshko, A.

Opt. Lett. 19(22): 1849-1851; Nov 15, 94

Waveguide Polarizers Processed by Localized Plasma Etching;

Veasey, D.L.; Larson, D.R.; Veigl, I.

Appl. Opt. 33(7): 1242-1244; Mar 1, 94 
Dielectric Materials and Devices

Integrated Optic Laser Emitting at 9051057 and 1356 nm;

Malone, K.J.; Sanford, N.A.; Hayden, J.S.; Sapak, D.L.

Proc., Topical Mtg. on Advanced Solid-State Lasers, Opt. Soc. Am., Feb 1-3, 1993, New Orleans, LA, 1-3, Post-Deadline; Aug 93

Integrated Optic Laser Emitting at 9061057 and 1358 nm;

Malone, K.J.; Sanford, N.A.; Hayden, J.S.

Electron. Lett. 29(8): 691-693; Apr 15, 93

Linewidth Narrowing in an Imbalanced Y-Branch Waveguide Laser;

Sanford, N.A.; Aust, J.A.; Malone, K.J.; Larson, D.R.

Opt. Lett. 18(4): 281-283; Feb 15, 93

Rare-Earth Doped Waveguide Lasers: The Potential for Compact Blue-Green Lasers;

Sanford, N.A.; Aust, J.A.; Malone, K.J.

Proc., 1992 Topical Mtg. on Compact Blue-Green Lasers, Opt. Soc. Am.,

Feb 20-21, 1992, Santa Fe, NM, 6:ThB4-1 to ThB4-4; 92

Rare-Earth-Doped Waveguide Devices;

Sanford, N.A.; Malone, K.J.; Aust, J.A.; Larson, D.R.

Tech. Dig., Opt. Soc. Am. Annual Mtg. 1992, Washington, DC, Vol 23, paper TuJ1; 92

Localized Plasma Etching for Device Optimization;

Larson, D.R.; Veasey, D.L.

J. Vacuum Sci. Tech. 10(1): 27-29; Dec 92

$\mathrm{Nd}: \mathrm{LiTaO}_{3}$ Waveguide Laser;

Sanford, N.A.; Aust, J.A.; Malone, K.J.; Larson, D.R.; Roshko, A.

Opt. Lett. 17(22): 1578-1580; Nov 15, 92

Direct Dispersion Measurement of Highly Erbium-Doped Optical Amplifiers Using a Low Coherence Reflectometer Coupled with Dispersive Fourier Spectroscopy;

Takada, K.; Kitagawa, K.; Hattori, K.; Yamada, M.; Horiguchi, M.; Hickernell, R.K. Electron. Lett. 28(20): 1889-1891; Sep 24, 92

Chromatic Dispersion Measurement of Erbium-Doped Fiber Amplifier Using Fourier Transform Spectroscopy;

Hickernell, R.K.; Takada, K.; Yamada, M.; Shimizu, M.; Horiguchi, M.

Tech. Dig., Optical Fiber Meas. Symp., in NIST SP 839, 201-204; Sep 92 
Extended-Cavity Operation of Glass Integrated-Optic Lasers: Mode-Locking Q-Switching and Wavelength Tuning;

Sanford, N.A.; Malone, K.J.; Larson, D.R.

Proc., Integrated Photonics Research Conf., Apr 9-11, 1991, Monterey, CA, Paper ThE3; 91

Integrated-Optic Waveguide Glass Lasers;

Sanford, N.A.; Malone, K.J.; Larson, D.R.

Proc., 1991 Optical Fiber Communications Conf., Feb 18-22, 1991, San

Diego, CA, 27; 91

Issues Affecting the Characterization of Integrated Optical Devices Subjected to Ionizing Radiation;

Hickernell, R.K.; Sanford, N.A.; Christensen, D.H.

Proc., SPIE, Vol 1474: 138-147; 91

Y-Branch Waveguide Glass Laser and Amplifier;

Sanford, N.A.; Malone, K.J.; Larson, D.R.; Hickernell, R.K.

Opt. Lett. 16(15): 1168-1170; Aug 1, 91

Extended Cavity Operation of Rare-Earth Doped Glass Waveguide Lasers;

Sanford, N.A.; Malone, K.J.; Larson, D.R.

Opt. Lett. 16(14): 1095-1097; Jul 15, 91

Waveguide Polarizers Using Hydrogenated Amorphous Silicon Claddings;

Veasey, D.L.; Hickernell, R.K.; Larson, D.R.; Batchman, T.E.

Opt. Lett. 16: 717-719; May 91

Integrated-Optic Laser Fabricated by Field-Assisted Ion-Exchange in Neodymium Doped Soda-Lime-Silicate Glass;

Sanford, N.A.: Malone, K.J.; Larson, D.R.

Proc.. 1990 Integrated Photonics Research Conf., Opt. Soc. Am., Mar 26-28, 1990. Hilton Head, SC, Vol 5, 114; 90

Photorefractive Instahilities in Proton-Exchanged Waveguides: Two-Wave Coupling and Chaos;

Sanford, N.A.; Hickernell, R.K.; Craig, R.M.

Proc.. 1990 Integrated Photonics Research Conf., Opt. Soc. Am., Mar 26-28, 1990, Hilton Head, SC, Vol 5, 91-92; 90 
Dielectric Materials and Devices

Semiconductor Claddings on Glass Channel Waveguides for Polarizers and Detectors;

Veasey, D.L.; Larson, D.R.; Phelan, R.J., Jr.; Batchman, T.E.

Proc., Opt. Soc. Am. Annual Mtg., Nov 4-9, 1990, Boston, MA, MA5; 90

In-Situ Optimization of Coupling between Semiconductor Claddings and Dielectric Waveguides;

Veasey, D.L.; Larson, D.R.; Batchman, T.E.

J. Appl. Phys. 68(7): 3753-3755; Oct 1, 90

Spatially Resolved Measurement of High Attenuation in Integrated Optic Polarizers;

Hickernell, R.K.; Veasey, D.L.; Aust, J.A.

Tech. Dig., 1990 Optical Fiber Meas. Symp., in NIST SP 792, 63-66; Sep 90

Integrated-Optic Laser Fabricated by Field-Assisted Ion Exchange in Neodymium Doped Soda-Lime-Silicate Glass;

Sanford, N.A.; Malone, K.J.; Larson, D.R.

Opt. Lett. 15(7): 366-368; Apr 90

Waveguide Loss Measurement Using Photothermal Deflection;

Hickernell, R.K.; Larson D.R.; Phelan, R.J., Jr.; Larson, L.E.

Appl. Opt. 27(13): 2636-2638; Jul 1, 88 


\section{SEMICONDUCTOR MATERIALS AND DEVICES}

Spatio-Spectral Mapping of Multimode Vertical Cavity Surface Emitting Lasers;

Knopp, K.J.; Christensen, D.H.; Vander Rhodes, G.; Pomeroy, J.M.; Goldberg, B.B.; Ünlü, M.S.

J. Lightwave Tech. 17(8): 1429-1435; Aug 99

High Bandwidth-Efficiency RCE Photodiodes Operating at 800-850 nm Wavelength;

Ünlü, M.S.; Ulu, G. ;Gökkavas, M.; Biyikli, N.; Kimukin, I.; Özbay, E.; Mirin, R.P.; Bertness, K.A.; Christensen, D.H.

Tech. Dig., OSA Ultrafast Electronics and Optoelectronics, Apr 14-16, 1999, Snowmass Village at Aspen, CO, UFB2-1/119-121; Apr 99

Photon Statistics of Pulsed, Vertical-Cavity, Surface-Emitting Lasers;

Beck, M.; Garrison, T.W.S.; Christensen, D.H.

Tech. Dig., OSA Quantum Optoelectronics, Apr 12-13, 1999, Snowmass

Village at Aspen, CO, QMD5-1/52-54; Apr 99

Spectral Mapping of Multimode Vertical-Cavity Surface-Emitting Lasers by Near-Field Scanning Optical Microscopy;

Knopp, K.J.; Christensen, D.H.; Vander Rhodes, G.; Pomeroy, J.M.; Goldberg, B.B.; Ünlü, M.S.

Proc., SPIE, Vol 3626, Testing, Packaging, Reliability, and Applications of

Semiconductor Lasers IV, Jan 28, 99, San Jose, CA: 208-216; Apr 99

High-Speed >90\% Quantum-Efficiency P-I-N Photodiodes with a Resonance Wavelength Adjustable in the 795-835 Range;

Özbay, E.; Kimukin, I.; Biyikli, N.; Aytür, O.; Gökkavas, M.; Ulu, G.; Ünlü, M.S.; Mirin, R.P.; Bertness, K.A.; Christensen, D.H.

Appl. Phys. Lett. 74(8): 1072-1074; Feb 22, 99

AllnP Benchmarks for Growth of AlGaInP Compounds by Organometallic Vapor-Phase Epitaxy;

Bertness, K.A.; Kurtz, S.R.; Asher, S.E.; Reedy, R.C., Jr.

J. Crystal Growth 196: 13-22; Jan 99

Cation Vacancy Formation and Migration in the AlGaAs Heterostructure System;

Mitev, P.; Seshardi, S.; Guido, L.J.; Schaafsma, D.T.; Christensen, D.H.

Appl. Phys. Lett. 73(25): 3718-3720; Dec 21, 98

Optical Constants of $\left(\mathrm{Al}_{0.98} \mathrm{Ga}_{0.02}\right)_{\mathrm{x}} \mathrm{O}_{\mathrm{y}}$ Native Oxides;

Knopp, K.J.; Mirin, R.P.; Christensen, D.H.; Bertness, K.A.; Roshko, A.; Synowicki, R.A.

Appl. Phys. Lett. 73(24): 3512-3514; Dec 14, 98 
Semiconductor Materials and Devices

High-Speed Widely-Tunable $>90 \%$ Quantum-Efficiency Resonant Cavity Enhanced p-i-n Photodiodes;

Biyikli, N.; Kimukin, I.; Aytür, O.; Gökkavas, M.; Ulu, G.; Mirin, R.P.; Christensen, D.H.; Ünlü, M.S.; Özbay, E.

Proc., IEEE Lasers and Electro-Optics Society, 11th Annual Mtg., Vol 1, Dec 14, 1998, Orlando, FL, 157-158; Dec 98

Characterization of InGaAs Quantum Dot Lasers with a Single Quantum Dot Layer as an Active Region;

Mirin, R.P.; Gossard, A.C.; Bowers, J.E.

Physics E, Elsevier Science 2(1-4): 738-742; Jul 498

High Bandwidth-Efficiency Resonant Cavity Enhanced Schottky Photodiodes for 800-850 nm Wavelength Operation;

Ünlü, M.S.; Gökkavas, M.; Onat, B.M.; Ata, E.; Özbay, E.; Mirin, R.P.; Knopp, K.J.; Bertness, K.A.; Christensen, D.H.

Appl. Phys. Lett. 72(21): 2727-2729; May 25, 98

Error Bars in Intensity and Phase Measurements of Ultrashort Laser Pulses;

Munroe, M.J.; Christensen, D.H.; Trebino, R.

Tech. Dig., Conf. on Lasers and Electro-Optics (CLEO '98), Vol6, May 3-8, 1998, San Francisco, CA, 462-463; May 98

Noise Reduction in Optical In Situ Measurements for Molecular Beam Epitaxy by Substrate Wobble Normalization;

Bertness, K.A.; Hickernell, R.K.; Hays, S.P.; Christensen, D.H.

J. Vacuum Sci.Tech. B 16(3): 1492-1497; May/Jun 1998

Pump Intensity Profiling of Vertical-Cavity Surface-Emitting Lasers Using Near-Field Scanning Optical Microscopy:

Vander Rhodes, G.H.; Pomeroy, J.M.; Ünlü, M.S.; Goldberg, B.B.; Knopp, K.J.; Christensen. D.H.;

Appl. Phys. Lett. 72(15): 1811-1813; Apr 13, 98

Spectral Broadening of Stochastic Light Intensity-Smoothed by a Saturated Semiconductor;

Munroe, M.J.; Cooper, J.; Raymer, M.G.

IEEE J. Quantum Elect. 34 (3): 548-551; Mar 98

Simultaneous Monitoring of Wafer and Environment-States During Molecular Beam Epitaxy;

Knopp, K.J.; Ketterl, J.R.; Christensen, D.H.; Pearsall, T.P.; Hill, J.R.

Proc., Mat. Res. Soc. Symp. on Thin Films-Structure and Morphology, December

2-6, 1996, Bosion, MA, Vol 441, 761-766; 97 
High Bandwidth-Efficiency GaAs Schottky Photodiodes for $840 \mathrm{~nm}$ Operation Wavelength; Ata, E.; Gökkavas, M.; Onat, B.M.; Islam, M.S.; Tuttle, G.; Mirin, R.; Knopp, K.J.; Bertness, K.A.; Christensen, D.H.; Ünlü, M.S.; Özbay, E.

Proc., 1997 Intl. Semiconductor Device Research Symp., Charlottesville, VA, Dec 11-13, 1997; Dec 97

Direct Measurement of Pump Intensity Distributions in an Optically Pumped Vertical-Cavity Surface-Emitting Laser;

Vander Rhodes, G.H.; Pomeroy, J.M.; Ulu, G.;Ünlü, M.S.; Goldberg, B.B.; Knopp, K.J.; Christensen, D.H.

Proc., IEEE Lasers and Electro-Optics Society, 10th Annual Mtg., Vol 1, Nov 1013, 1997, San Francisco, CA, 285-286; Nov 97

Wobble Normalization for Optical In Situ Measurements in Molecular Beam Epitaxy;

Bertness, K.A.; Hickernell, R.K.; Christensen, D.H.

Tech. Prog., 16th Annual North American Conf. on Molecular Beam Epitaxy, Oct 5-8, 1997, Ann Arbor, MI; Oct 5, 97

Characterization and Modeling of InGaAs Quantum Dot Lasers;

Mirin, R.E.; Gossard, A.; Bowers, J.

Proc., 8th Intl. Conf. on Modulated Semiconductor Structures, Santa Barbara, CA; Aug 97

Ultrafast Optical Excitation of Vertical-Cavity Surface-Emitting Lasers;

Munroe, M.; Knopp, K.J.; Christensen, D.H.

Tech. Dig., Quantum Electronics and Laser Science Conf. (QELS '97), Vol 12, May 18-23, 1997, Baltimore, Maryland, 49; May 97

Mode Splitting in Vertical-Cavity Microlasers from Side-Emission Measurements;

Schaafsma, D.; Christensen, D.H.

Tech. Dig., Conf. on Lasers and Electro-Optics (CLEO '97), Vol 11, May 18-23, 1997, Baltimore, Maryland, 271-272; May 97

Vertical-Cavity Surface-Emitting Lasers with Low-Ripple Optical Pumping Windows; Knopp, K.J.; Christensen, D.H.; Hill, J.R.

IEEE J. Quantum Elect. (Selected Topics) 3(2): 366-371; Apr 97

Evaluating Epitaxial Growth Stability;

Christensen, D.H.; Hill, J.R.; Hickernell, R.K.; Matney, K.; Goorsky, M.S. Materials Science \& Engineering B44(1-3): 113-116; Feb 97 
Semiconductor Materials and Devices

Cavity Coupling in Vertical-Cavity Semiconductor Lasers;

Schaafsma, D.T.; Christensen, D.H.

NISTIR 5047; 135 pp; Jan 97

Vertical-Cavity Surface-Emitting Lasers with Low-Ripple Optical Pump Bands;

Knopp, K.J.; Christensen, D.H.; Hill, J.R.

Appl. Phys. Lett. 69(26): 3987-3989; Dec 23, 96

Thin-Film Design for Enhanced Stability of Optically Pumped Vertical-Cavity Surface-Emitting Lasers;

Knopp, K.J.; Christensen, D.H.; Hill, J.R.; Masterson, K.D.

Surface and Coatings Tech. 86-87: 783-787; Dec 96

Mode Splitting in Side Emission from Vertical-Cavity Surface-Emitting Lasers;

Schaafsma, D.T.; Christensen, D.H.

Phys. Rev. B 54(20): 14618-14622; Nov 15, 96

Cross-Sectional Photoluminescence and its Application to Buried-Layer Semiconductor Structures;

Schaafsma, D.T.; Christensen, D.H.

J. Appl. Phys. 78(2): 694-699; Jul 15, 95

Correlation of Optical, X-Ray, and Electron Microscopy Measurements on Semiconductor Multilayer Structures;

Christensen, D.H.; Hickernell, R.K.; Schaafsman, D.T.; Pellegrino, J.G.; McCollum, M.J.; Hill, J.R.; Rai, R.S.

Proc., SPIE, Vol 2141: 177-188; 94

Measurement and Simulation of Photoluminescence Spectra from Vertical-Cavity Quantum-Well Laser Structures;

Schaafsma, D.T.; Hickernell, R.K.; Christensen, D.H.

Proc., SPIE, Vol 2139: 92-102; 94

Comparative Photoluminescence Measurement and Simulation of Vertical-Cavity Semiconductor Laser Structures;

Schaafsma, D.T.; Christensen, D.H.; Hickernell, R.K.; Pellegrino, J.G.

Proc., Mat. Res. Soc. Symp. on Semiconductor Heterostructures, Nov 29-Dec 2, 1993, Boston, MA, Vol 326, 483-488; Jun 94

Determination of the Complex Refractive Index of Individual Quantum Wells from Distributed Reflectance;

Hickernell, R.K.; Christensen, D.H.; Pellegrino, J.G.; Wang, J.; Leburton, J.P.

J. Appl. Phys. 75(6): 3056-3059; Mar 15, 94 
Vertical-Cavity Semiconductor Lasers: Structural Characterization, CAD, and DFB Structures; Christensen, D.H.; Parsons, C.A.; Pellegrino, J.G.; Hill, J.R.; Rai, R.S.; Crochiere S.M.; Hickernell, R.K.; Schaafsma, D.T.

Proc., SPIE, Vol 1850: 115-121; 93

Vertical-Cavity Semiconductor Structures: Materials Characterization; Christensen, D.H.; Hill, J.R.; Schaafsma, D.T.; Hickernell, R.K.; Pellegrino, J.G.; Tseng, W.F.

Tech. Dig., Conf. on Lasers and Electro-Optics (CLEO '95), Vol 11, May 2-7, 1993, Baltimore, MD, 234-235; 93

Vertical-Cavity Optoelectronic Structures: CAD, Growth, and Structural Characterization;

Christensen, D.H.; Crochiere, S.M.; Pellegrino, J.G.; Rai, R.S.; Parsons, C.A.; Tseng, W.F.; Hickernell, R.K.

Proc., Mater. Res. Soc. Symp. on Semiconductor Heterostructures for Photonic and Electronic Applications, Nov 30-Dec 4, 1992, Boston, MA, Vol 281: 307$312 ; 93$

Characterization of Vertical-Cavity Semiconductor Structures;

Christensen, D.H.; Crochiere, S.M.; Parsons, C.A.; Pellegrino, J.G.; Hickernell, R.K.; Rai, R.S.

J. Appl. Phys. 72(12): 5982-5989; Dec 15, 92

Mean Lifetime Calculations of Quantum Well Structures: A Rigorous Analysis;

Ghatak, A.K.; Goyal, I.C.; Gallawa, R.L.

IEEE J. Quant. Electron. QE-26(2): 305-310; Feb 90

InSb-GaAsP Infrared to Visible Light Converter;

Phelan, R.J., Jr.

Proc., IEEE, Vol 55: 1505-1502; Aug 67

InSb MOS Infrared Detector;

Phelan, R.J., Jr.; Dimmock, J.O.

Appl. Phys. Lett. 10: 55-57; Jan 67

Incoherent Source Optical Pumping of Visible and Infrared Semiconductor Lasers;

Phelan, R.J., Jr.

Proc., IEEE, Vol 54: 1119-1120; Aug 66

Laser Emission by Optical Pumping of Semiconductors;

Phelan, R.J., Jr.

Proc., Quantum Electronics Conf., 435-441; Jul 65 


\section{CONFERENCE DIGESTS AND PROCEEDINGS}

Technical Digest-Symposium on Optical Fiber Measurements, 1998;

Day, G.W.; Franzen, D.L.; Williams, P.A., eds.

NIST SP 930, 187 pp; Sep 98

Technical Digest-Symposium on Optical Fiber Measurements, 1996;

Day, G.W.; Franzen, D. L.; Williams, P. A., eds.

NIST SP 905, 215 pp; Oct 96

Technical Digest-Conference on Precision Electromagnetic Measurements;

Young, M.; Cook, R.J., eds.

IEEE Trans. Instrum. Meas. IM-44(2); Apr 95

Technical Digest-Symposium on Optical Fiber Measurements, 1994;

Day, G.W.; Franzen, D.L.; Hickernell, R.K., eds

NIST SP 864, 219 pp; Sep 94

Technical Digest-Conference on Precision Electromagnetic Measurements;

Steele, J.M.; Young, M., eds

IEEE Trans. Instrum. Meas. IM-42(2): 679 pp; Apr 93

Technical Digest-Symposium on Optical Fiber Measurements, 1992;

Day, G.W.; Franzen, D.L., eds

NIST SP 839, 245 pp; 92

Laser Induced Damage in Optical Materials: 1989;

Bennett, H.E.; Chase, L.L.; Guenther, A.H.; Newnam, B.E.; Soileau, M.J. NIST SP 801,670 pp; 90

Technical Digest-Symposium on Optical Fiber Measurements, 1990;

Day, G.W.; Franzen, D.L., eds.

NIST SP 792; Sep 90

Laser Induced Damage in Optical Materials: 1988;

Bennett, H.E.; Guenther, A.H.; Newnam, B.E.; Soileau, M.J.

NIST SP 775, $576 \mathrm{pp} ; 89$

Surface Characterization and Testing II;

Grievenkamp, J.E.; Young, M., eds.

Proc., SPIE, Vol 1164, Surface Characterization and Testing II, Aug 10-11, 1989,

San Diego, CA: 271 pp; 89 
Laser Induced Damage in Optical Materials: 1987;

Bennett, H.E.'; Guenther, A.H.; Milam, D.; Newnam, B.E. NIST SP 756, 650 pp; 88

Laser Induced Damage in Optical Materials: 1986;

Bennett, H.E.; Guenther, A.H.; Milam, D.; Newnam, B.E. NBS SP 752, 724 pp; 88

Technical Digest-Symposium on Optical Fiber Measurements, 1988;

Day, G.W.; Franzen, D.L., eds.

NBS SP 748, 202 pp; Sep 88

Laser Induced Damage in Optical Materials: 1985;

Bennett, H.E.; Guenther, A.H.; Milam, D.; Newnam, B.E. NBS SP 746, 576 pp; Jul 88

Laser Induced Damage in Optical Materials: 1984;

Bennett, H.E.; Milam, D.; Guenther, A.; Newnam, B.E.

Proc., 16th Annual Symp. on Optical Materials for High Power Lasers, Oct 15-17, 1984, Boulder, CO, NBS SP 727; Oct 86

Technical Digest-Symposium on Optical Fiber Measurements, 1986;

Day, G.W.; Franzen, D.L., eds.

NBS SP 720, 149 pp; Sep 86

Laser Induced Damage in Optical Materials: 1983;

Bennett, H.E.; Milam, D.; Guenther, A.; Newnam, B.E.

NBS SP 688, 581 pp; Nov 85

Technical Digest-Symposium on Optical Fiber Measurements, 1984;

Day, G.W.; Franzen, D.L., eds.

NBS SP-683; Oct 84

Fiber Optics: Short-Haul and Long-Haul Measurements and Applications;

Gallawa, R.L.ed.

Proc., SPIE, Vol 355; 83

Technical Digest-Symposium on Optical Fiber Measurements, 1982;

Franzen, D.L.; Day, G.W.; Gallawa, R.L., eds.

NBS SP 641; Oct 82 
Conference Digests and Proceedings

Technical Digest-Symposium on Optical Fiber Measurements, 1980;

Day, G.W.; Franzen, D.L., eds.

NBS SP 597; Oct 80 


\section{MISCELLANEOUS TOPICS}

Optoelectronics at NIST;

Day, G.W., ed.

$$
\text { NISTIR 5054; } 49 \text { pp; Sep } 96
$$

Optoelectronics at NIST;

Day, G.W, ed.

Proc., IEEE Lasers and Electro-Optics Society, 8th Annual Mtg, Vol 2, Nov 1-2, 1995, San Francisco, CA, 73-74; Nov 95

Comment on the Paper "Etalon Effect in Laser Mirrors";

Young, $\mathrm{M}$.

Opt. Eng. 34(4): 1243; Apr 95

Guest Editorial for the IEEE Transactions on Instrumentation and Measurement;

Young, $\mathrm{M}$.

IEEE Trans. Instrum. Meas. IM-44(2): 83-84; Apr 95

Spatial Light Modulator for Texture Classification;

Haggerty, J.; Young, $M$.

Appl. Opt. 28(23): 4992-4995; Dec 1, 89

The Pinhole Camera;

Young, $\mathrm{M}$.

The Physics Teacher, 648-655; Dec 89

Fresnel Lenses Display Inherent Vignetting;

Young, $\mathrm{M}$.

Appl. Opt. 27(17): 3593-3594; Sep 1, 88

Cool It!;

Lehman, J.H.

Science Teacher, 29-32; Mar 88

Microscope Objectives, Cover Slips, and Spherical Aberration;

Oates, C.W.; Young, M.

Appl. Opt. 26(11): 2043; Jun 1, 87

Hybrid Computer-Optical Processing with Inexpensive Liquid Crystal Television;

Young, M.; Weppner, M.

Proc., SPIE, Vol 700: 146-153; 86 
Scratch-and-Dig Standard Revisited;

Young, $\mathrm{M}$.

Appl. Opt. 25(12): 1922-1928; Jun 86

Fiber Bandwidth Measurement Using Pulse Spectrum Analysis;

Yang, S.; Gallawa, R.L.

Appl. Opt. 25(7): 1069-1070; Mar 86

Low-Cost LCD Video Display for Optical Processing;

Young, $\mathrm{M}$.

Appl. Opt. 25(7): 1024-1026; Apr 86

Scratch Standards Should Not be Used to Predict Damage Threshold;

Young, $\mathrm{M}$.

NBS SP $669,151-156 ; 85$

The Scratch Standard is Not a Performance Standard;

Young, $M$.

Dig., Workshop on Optical Fabrication and Testing, Jun 12-13, 1985, Cherry Hill, NJ, ThAA4-1and2; 85

Tunable Scratch Standards;

Young, M.; Johnson, E.G., Jr.; Goldgraben, R.

Proc., SPIE, Vol 525: 70-77; 85

The Scratch Standard is Only a Cosmetic Standard;

Young, $\mathrm{M}$.

Laser Focus/Electro-Optics, 138-140; Nov 85

Pattern Recognition Using Incoherent OTF Synthesis and Edge Enhancement;

Katzir, Y.; Young, M.; Glaser, I.

Appl. Opt. 24(6): 863-867; Mar 15, 85

Redefining the Scratch Standards;

Young, M.; Johnson, E.G., Jr.

NBS TN1080, 20 pp; Feb 85

Some Trends in Optical Electronic Metrology;

Sanders, A.A.

Proc., 1984 Measurement Science Conf., Los Angeles, CA; Jan 84 
Objective Measurements and Characteristics of Scratch Standards;

Young, $\mathrm{M}$.

Proc., SPIE, Vol 3526: 86-92; Spring 83

Simulating the Scratch Standards for Optical Surfaces-Theory;

Johnson, E.G., Jr.

Appl. Opt. 22(24): 4056-4068; Dec 83

Linewidth Measurement by High-Pass Filtering-A New Look;

Young, $M$.

Appl. Opt. 22(13): 2022-2025; Jul 83

Questions Students Ask;

Young, $\mathrm{M}$.

Physics Teacher, 194-195; Mar 83

Quantum Noise Limits the Pinspeck Camera to Simple Objects;

Young, $\mathrm{M}$.

J. Opt. Soc. Am. 72(3): 402-403; Mar 82

A Measurement Method for Determining the Optical and Electro-Optical Properties of a Thin Film;

Larson, D.L.

NBSIR 81-1652; Dec 81

Book Review: Linear Systems Fourier Transforms and Optics by J.D. Gaskill;

Young, $\mathrm{M}$.

J. Opt. Soc. Am. 69: 637-638; 79

Conference on Optical Scattering Standards;

Young, $\mathrm{M}$.

Proc., SPIE, Vol 181: 133-134; Apr 79

Linear and Nonlinear Optical Properties of Trigonal Selenium;

Day, G.W.

Appl. Phys. Lett. 18: 347-349; Apr 71

Some Optical Properties of Cesium Cupric Chloride;

Day, G.W.; Gruzensky, P.M.

Appl. Opt. 9: 2494-2795; 70 


\section{AUTHOR INDEX}

Alpert, B.K. . . . . . . . . . . . . . . . . . . . . . . . . . . . 29

Alvarez, R. . . . . . . . . . . . . . . . . . . 36

Amin, J. . . . . . . . . . . . . . . . . . . . . . . . . 63-65

Anderson, M.H. . . . . . . . . . . . . . . . . . . . 11

Andrews, J.R. . . . . . . . . . . . . . . . . . . . . . 42

Asher, S.E. . . . . . . . . . . . . . . . . . . . . . . 69

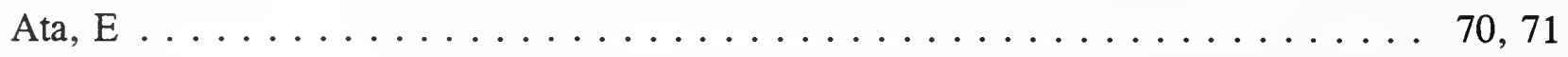

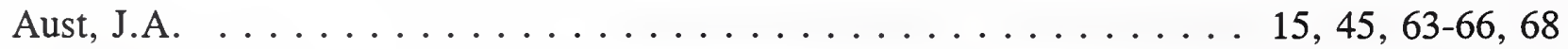

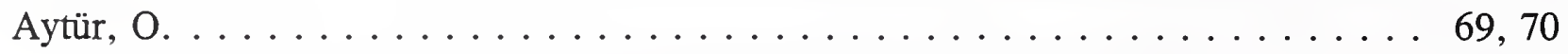

Baines, J. . . . . . . . . . . . . . . . . . . . . . . 34

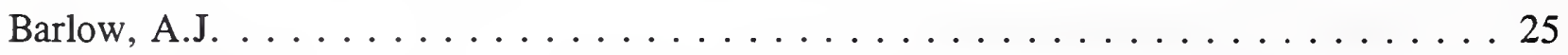



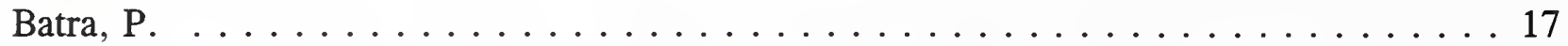

Beahn, T.J. . . . . . . . . . . . . . . . . . . . 52

Beck, M. . . . . . . . . . . . . . . . . . . . . . . 69

Beers, Y. . . . . . . . . . . . . . . . . 21

Bendett, M. . . . . . . . . . . . . . . . . . 63

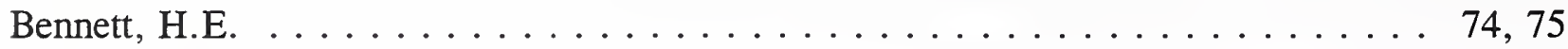

Bertness, K.A. . . . . . . . . . . . . . . . . . . . 69-71

Biyikli, N. . . . . . . . . . . . . . . . . . . 69, 70

Bloom, L.R. . . . . . . . . . . . . . . . . . . . . 39, 42

Bluhm, H. . . . . . . . . . . . . . . . . . . . . . 64

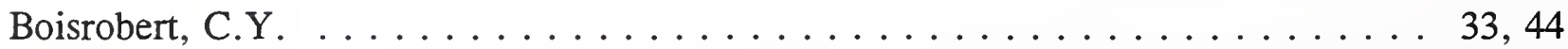

Bollinger, J.J. . . . . . . . . . . . . . . . . . . . . . . . . . . 61



Brasunas, J.C. . . . . . . . . . . . . . . . . . . . . . 45

Brilliant, N.A. . . . . . . . . . . . . . . . . 26, 29

Bruno, T.J . . . . . . . . . . . . . . . . . . . 47

Case, W.E. . . . . . . . . . . . . . . . . . . . . 18-20, 22, 23

Cernosek, R.W. . . . . . . . . . . . . . . . . . . . . . 5 57, 58

Chamberlain, G.E. . . . . . . . . . . . . 20, 37, 38, 40, 42

Chandler, G.I. . . . . . . . . . . . . . . . . . . . . . . . 57, 58

Chase, L.L. . . . . . . . . . . . . . . . . . . . . . . . 74

Cherin, A.H. . . . . . . . . . . . . . . . . . . . . . . . . 34

Christensen, D.H. . . . . . . . . . . . . . . 44, 67, 69-73





Clarke, I. . . . . . . . . . . . . . . . . . . 47, 48, 50, 51

Clement, T.S. . . . . . . . . . . . . . . . . . . . 9 
Collins, G.J. 14,21

Conrad, D. 49,57

Cook, A.R. $21,23,24$

Cook, R.J. 74

Cooper, J. 11,70

Craig, R.M. $11,18,46,47,52,59,67$

Crochiere, S.M. 73

Cromer, C.L.

Danielson, B.L. $14,21,23,27,30,33-35,37-42,44$

Das, U.K. 32

Day, G.W. $13,14,20-24,30,33,35,37-42,47-58,67,74-77,79$

Deeter, M.N. $48-57,67$

Diddams, S.A.

Dimmock, J.O.

Dowell, M.L.

Drapela, T.J. $25,27-29,31,32,34,61$

Dyer, S.D. 46,47

Eaton, H.E.

Engelsrath, A 35,37

Esman, R.D.

Espejo, R.J. 46

Etzel, S.M. $12,46,48,55,57,58$

Evans, C. 64

Evenson, K.M. $14,23,24$

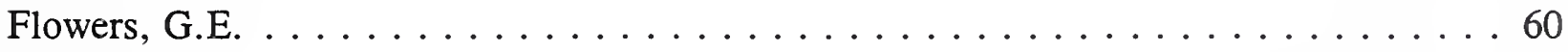

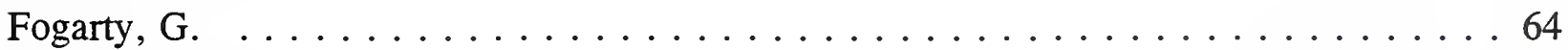

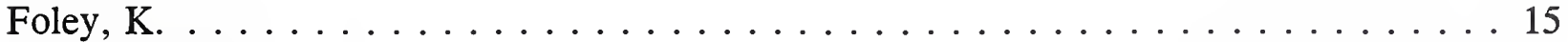

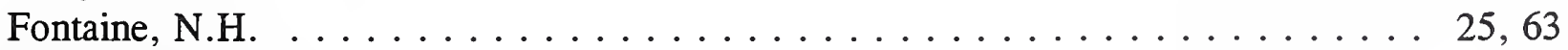

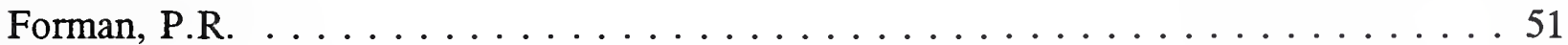

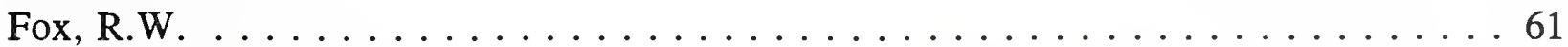

Franzen, D.L. . . . . . . . . . . . 10, 12-14, 21-23, 25-42, 44, 61, 74-76

Fratello, V.J. $52-54$

Frederick, N.V. 12,13

Funk, D.S.

Gallagher, M.D. 
Author Index

Gary, J.M. . . . . . . . . . . . . . . . . . . . . . . . . 64

Geist, J. . . . . . . . . . . . . . . . . . . . . . . 23



Gifford, A.D. . . . . . . . . . . . . . . . . . . . 10

Gilbert, S.L. . . . . . . . . . . . . . . . . . . . . . . 59-62

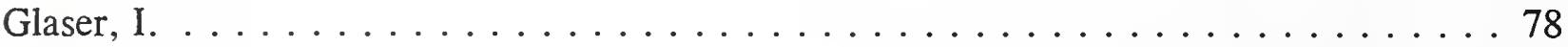

Gökkavas, M. . . . . . . . . . . . . . . . . . . . . . 69-71

Goldberg, B.B. . . . . . . . . . . . . . . . . . . . . . 69-71

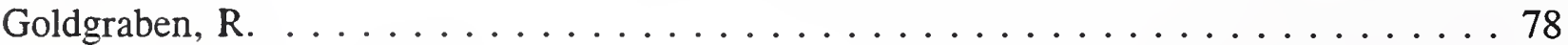

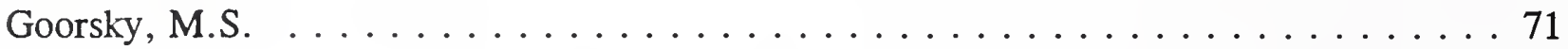

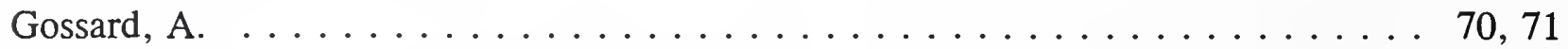

Goyal, I.C. . . . . . . . . . . . . . . . . . 28-33, 43-45, 73

Gray, E.M. . . . . . . . . . . . . . . . . . . . 39, 42

Grievenkamp, J.E. . . . . . . . . . . . . . . . . . . . . . 74

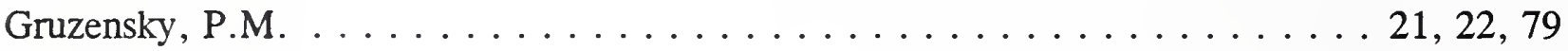

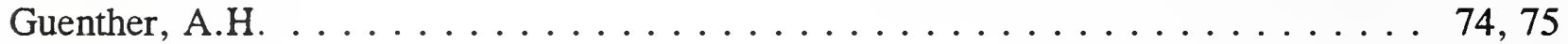

Guido, L.J. . . . . . . . . . . . . . . . . . . . . . . 69

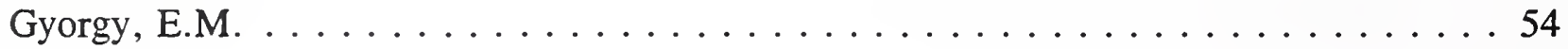



Haggerty, J. . . . . . . . . . . . . . . . . . . . . . . . . 77

Hale, P.D. . . . . . . . . . . . 9, 10, 28, 30-33, 47-49, 57, 59

Halford, D. . . . . . . . . . . . . . . . . . 24

Hamilton, C.A. . . . . . . . . . . . . . . 21, 22

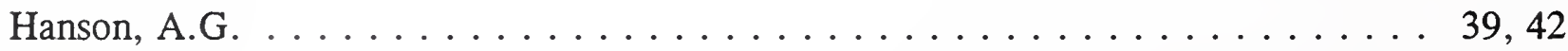

Hattori, K. . . . . . . . . . . . . . . . . . . . . . . 66

Hayden, J.S. . . . . . . . . . . . . . . . . 63, 65, 66

Hays, S.P. . . . . . . . . . . . . . . . . . . . . 70

Head, E.D. . . . . . . . . . . . . . . . . . . . . . . 34

Heinzen, D.J. . . . . . . . . . . . . . . . . . . . . . 61

Hernday, P.R. . . . . . . . . . . . . . . . . . . . . . . . 27

Hickernell, R.K. . . . . . . . . . . . . 30, 45, 51, 66-68, 70-74

Hill, J.R. . . . . . . . . . . . . . . . . . . . . . . . . . . . 70-73

Hjelme D.R. . . . . . . . . . . . . . . . . . . . . . . . . . . 19

Hollberg, L. . . . . . . . . . . . . . . . . . . . . . . 61

Horiguchi, M. . . . . . . . . . . . . . . . . . 30, 66

Houde-Walter, S.N. . . . . . . . . . . . . . . . . . . . 63

Humphreys, D.A. . . . . . . . . . . . . . . . . . . 10

Ishizuka, S. . . . . . . . . . . . . . . . . . . 46

Islam, M.S. . . . . . . . . . . . . . . . . . . . . . . 71

Itano, W.M. . . . . . . . . . . . . . . . . . . 61 


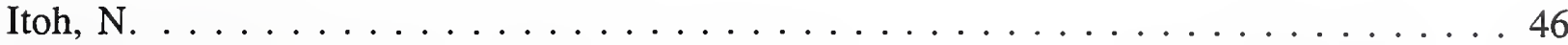

Iwashita, I. . . . . . . . . . . . . . . . . . . . . . . . . . . 10

Januar, I.P . . . . . . . . . . . . . . . . . . . . . . . . . . . . . . . . . . 19

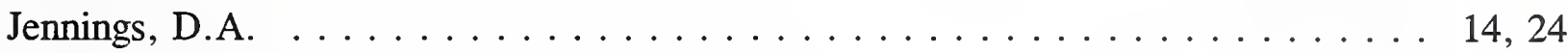

Jindal, R. . . . . . . . . . . . . . . . . . . . 43

Jinno, M. . . . . . . . . . . . . . . . . . . . . . . . . . . . . . . 29

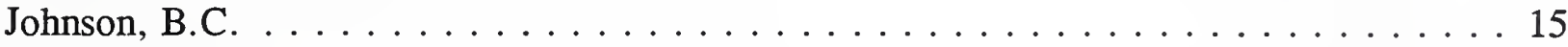

Johnson, E.G., Jr. . . . . . . . . . . . . . . . . . 11-13, 19-21, 78, 79

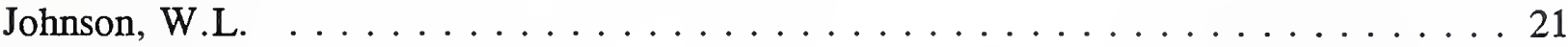

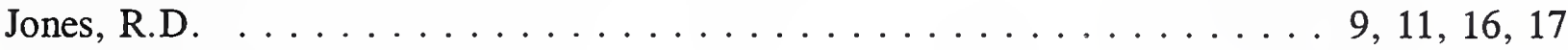

Juneau, R.I. . . . . . . . . . . . . . . . . . . . . . . . . . . 33

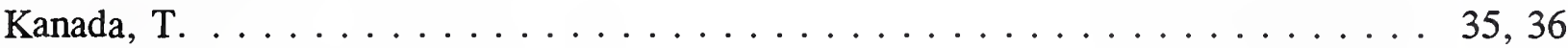

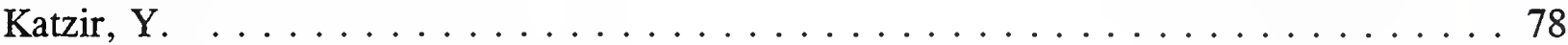

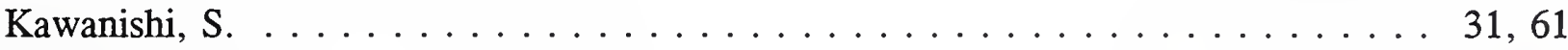

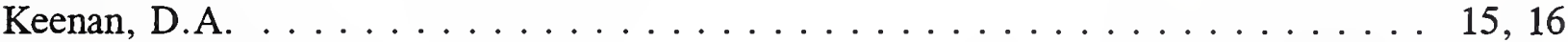

Ketterl, J.R. . . . . . . . . . . . . . . . . . . . . . . . . . 70

Kim, E.M. . . . . . . . . . . . . . . . . . . . 35, 38-41

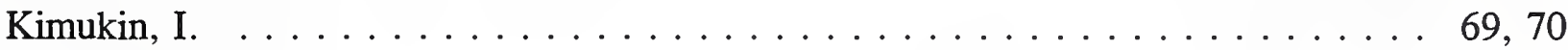

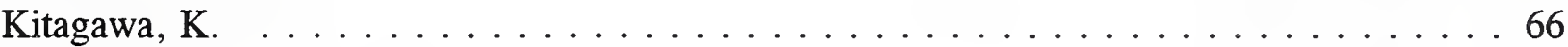

Klein, G.P. . . . . . . . . . . . . . . . . . . . . . . . . 22

Knopp, K.J. . . . . . . . . . . . . . . . . . . . . . . . . . . . . 69-72

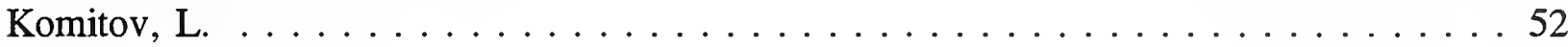

Kreger, S.T. . . . . . . . . . . . . . . . . . . . 46

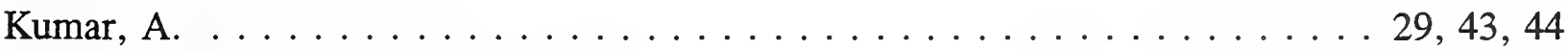

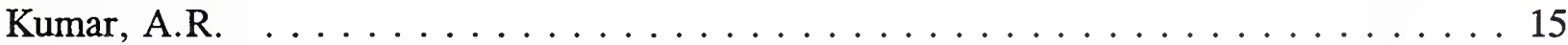

Kurtz, S.R. . . . . . . . . . . . . . . . . . . . . . . . . . . 69

Larson, D.R. . . . . . . . . . . . . . . . . 9-13, 17-19, 37, 45, 65-68, 79

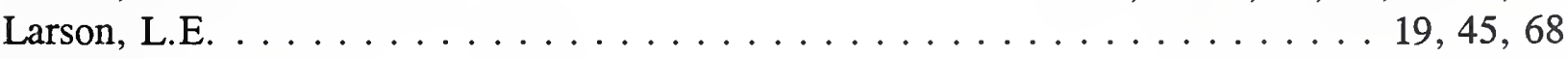

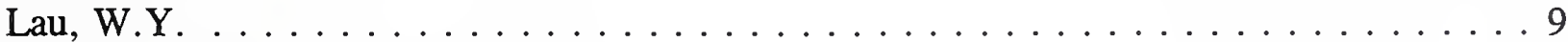

Leburton, J.P. . . . . . . . . . . . . . . . . . . . . . . . . . . . . . . 72

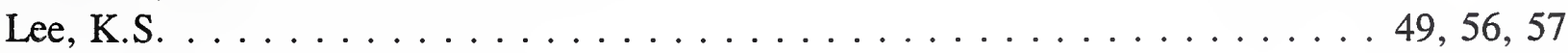

Lehman, J. . . . . . . . . . . . . . . . . . . . . . . . . 15, 17,77

Leonhardt, R.W. . . . . . . . . . . . . . . . . . . . 9, 10, 15

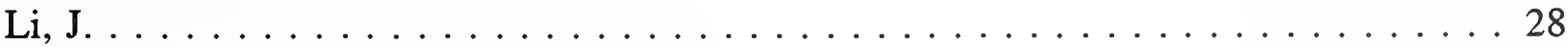

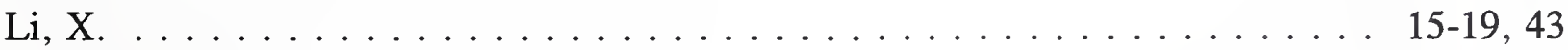

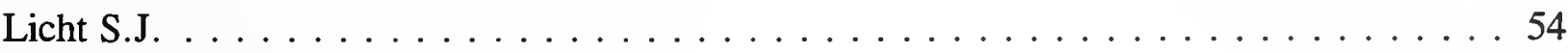

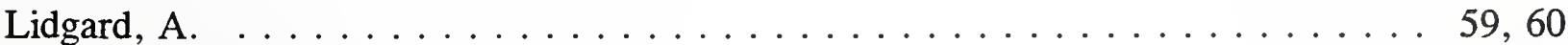

Lieberman, R.A. . . . . . . . . . . . . . . . . . . . . . 54

Livigni, D.J. . . . . . . . . . . . . . . . . . . . . . . . . . 15-18 
Author Index

Lovely, P.S. . . . . . . . . . . . . . . . . . . . . 51

Lowekamp, J.B. . . . . . . . . . . . . . . . . . . . . . . . . . 64

Mackechnie, C. . . . . . . . . . . . . . . . . . . . . 25

Mahler, R.J. . . . . . . . . . . . . . . . . . . . . 23, 24

Maisonneuve, J.M. . . . . . . . . . . . . . . . . . . . 12, 36

Malone, K.J. . . . . . . . . . . . . . . . . . . . . . . . 65-68

Mamileti, L. . . . . . . . . . . . . . . . . . . . . . . 32

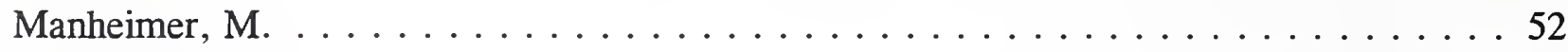

Marquardt, J.H. . . . . . . . . . . . . . . . . . . 61

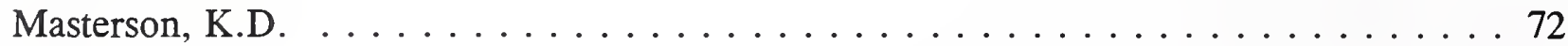

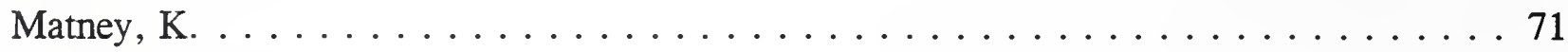

McCollum, M.J. . . . . . . . . . . . . . . . . . . . . . . 72

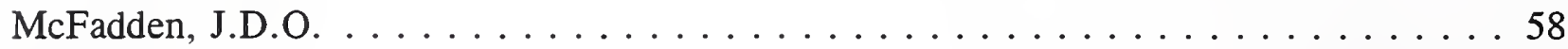

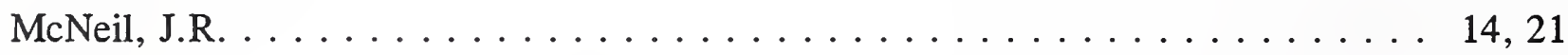

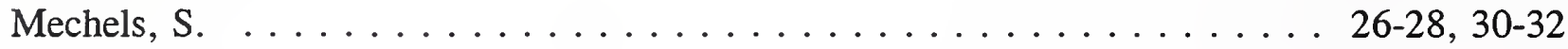

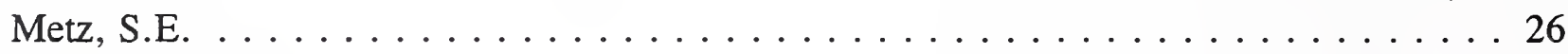

Mickelson, A.R. . . . . . . . . . . . . . . . . . . . . . 19

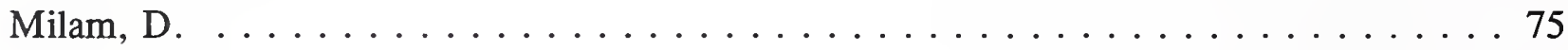

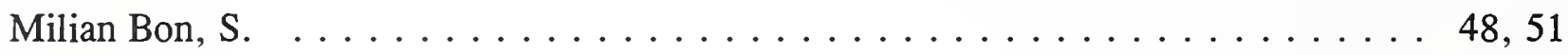

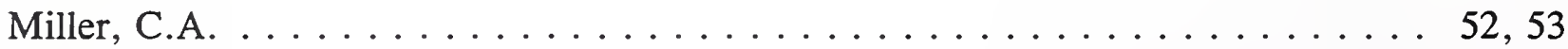

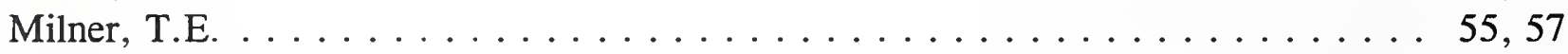

Minemoto, H. . . . . . . . . . . . . . . . . . . 46

Mirin, R.P. . . . . . . . . . . . . . . . . . 69-71

Mitev, P. . . . . . . . . . . . . . . . . . . . . . . . 69

Morris-Hotsenpiller, P.A. . . . . . . . . . . . . . . . . . . . 64

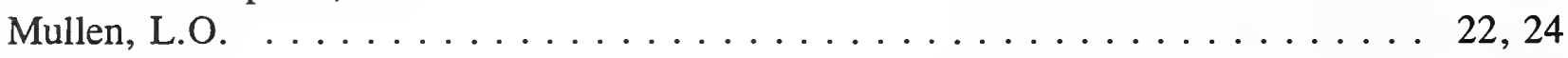

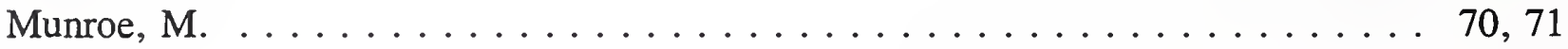

Nam, D. . . . . . . . . . . . . . . . . . . . . 64

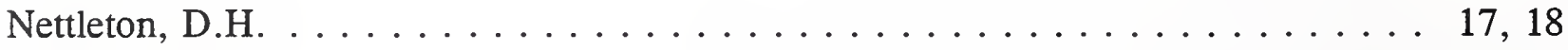

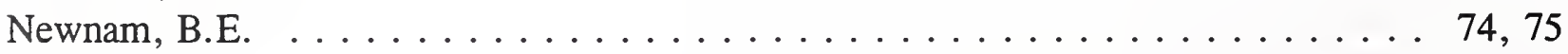

Novak, S.W. . . . . . . . . . . . . . . . . . 65

Oates, C.W. . . . . . . . . . . . . . . . . 34, 77

Obarski, G.E. . . . . . . . . . . . . . . . . 9-11, 32, 62

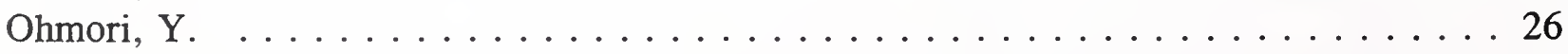

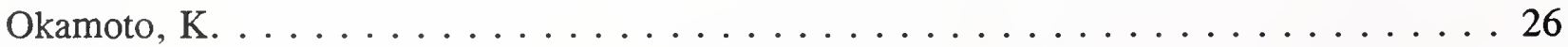

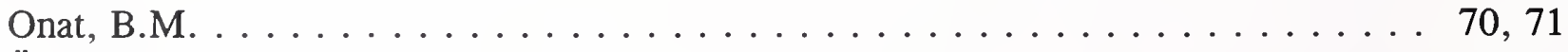

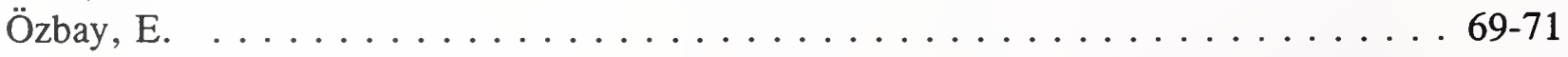

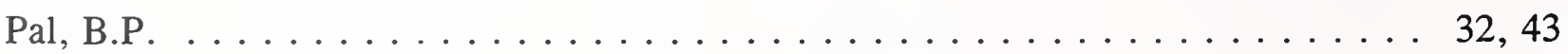




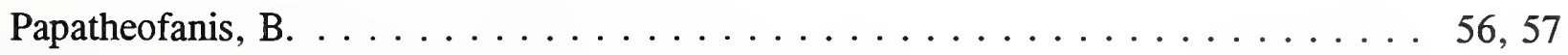

Park R. . . . . . . . . . . . . . . . . . 9

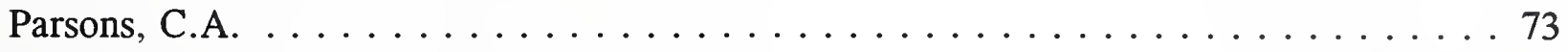

Patrick, H. . . . . . . . . . . . . . . . . . . . . . 59-61

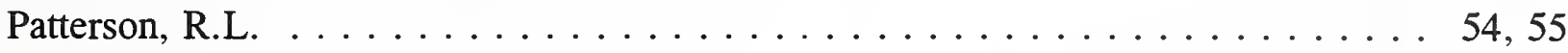

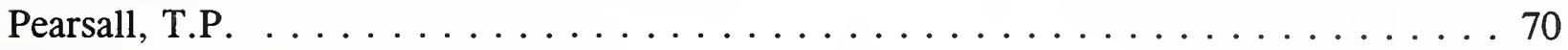

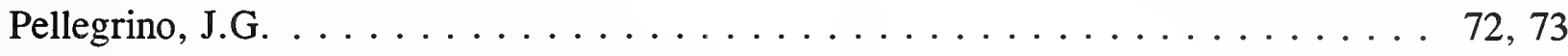

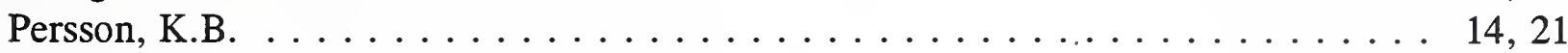

Peters, P.M. . . . . . . . . . . . . . . . . . . 63

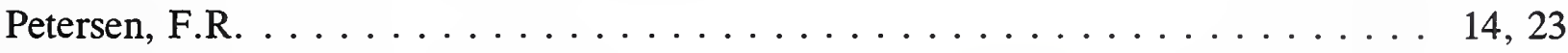

Peterson, R.L. . . . . . . . . . . . . . . . . . . . . . . 22

Phelan, R.J., Jr. . . . . . . . . . . . . . 9-13, 17-19, 21-24, 35, 37, 45, 68, 73

Pomeroy, J.M. . . . . . . . . . . . . . . . . . . . . . . . 69-71

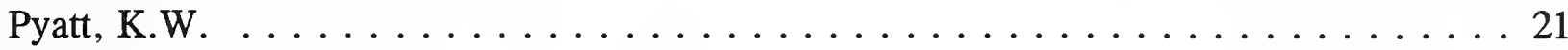

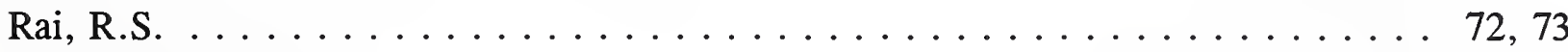

Raine, K. . . . . . . . . . . . . . . . . . . . . . . . 34

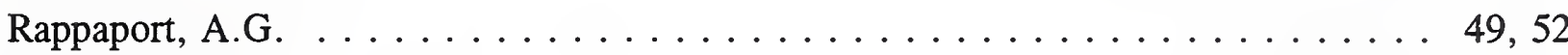

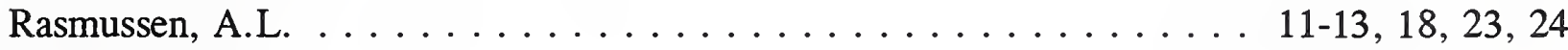

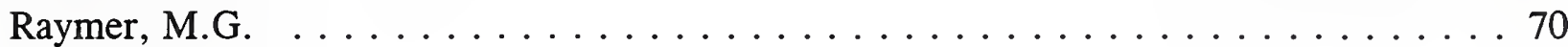

Reedy, R.C., Jr . . . . . . . . . . . . . . . . . . . . . . . . . . 69

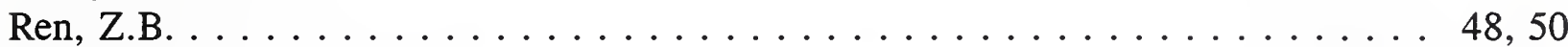

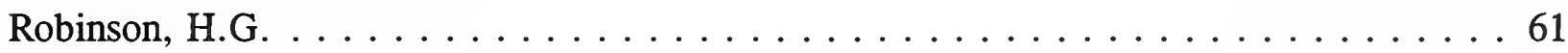

Rochford, K.B. . . . . . . . . . . . . . . . . . . . . 46-52

Rockwell, R.J., Jr. . . . . . . . . . . . . . . . . . . . . . . . . . . . . 17

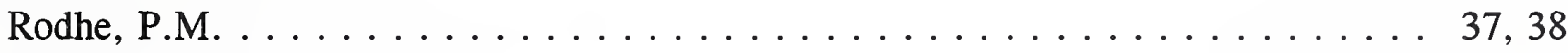

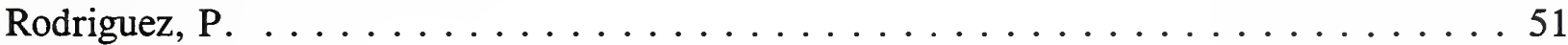

Rohrer, G.S. . . . . . . . . . . . . . . . . . . . . . . . . . 64



Rose, A.H. . . . . . . . . . . . . . . . . . 26, 46-57, 67

Roshko, A. . . . . . . . . . . . . . . . . . . . . 64-66, 69

Roy, S. . . . . . . . . . . . . . . . . . 31, 44

Russell, T.W. . . . . . . . . . . . . . . . . . 23

Samuelson, S. . . . . . . . . . . . . . . . . . . . . . . . . 54

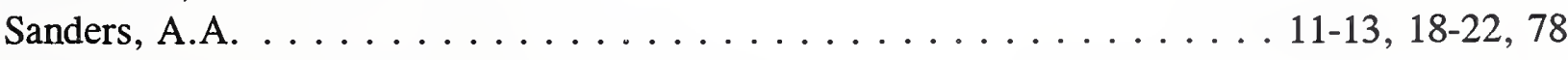

Sanford, N.A. . . . . . . . . . . . . . . . . . . . . . . 63-68

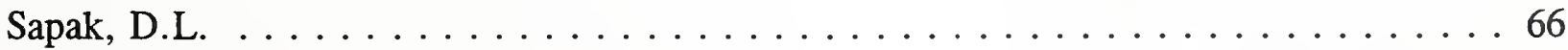

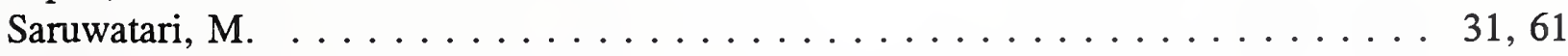

Sauvageau, J. . . . . . . . . . . . . . . . . . . . . . 15

Schaafsma, D.T. . . . . . . . . . . . . . . . . 69, 71-73

Schlager, J.B. . . . . . . . . . . . . . . . . . . . . 25-33, 61 
Author Index

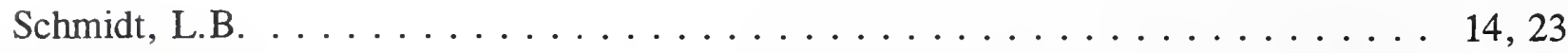



Seshardi, S. . . . . . . . . . . . . . . . . . . . . . . . . . . 69

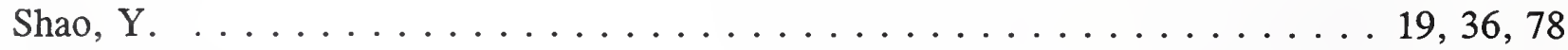

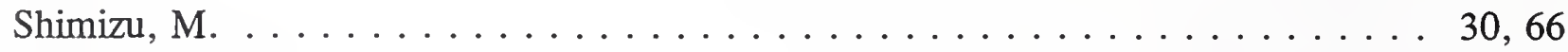

Simmons, W.R. . . . . . . . . . . . . . . . . . . . . 24

Simpson, P.A. . . . . . . . . . . . . . . 11-13, 20

Smith, R.J. . . . . . . . . . . . . . . . . . . . . . 34

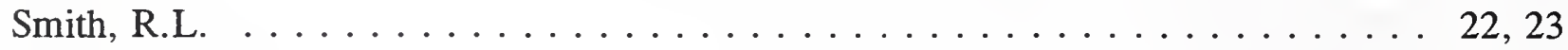

Smith, S.J. . . . . . . . . . . . . . . . . . . 11

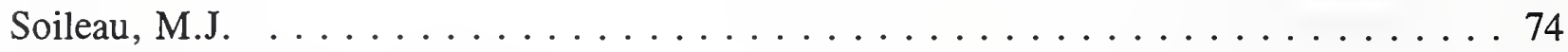

Srivastava, R. . . . . . . . . . . . . . . . . . . 37





Stephens, E.F. . . . . . . . . . . . . . . . . . . . . 59

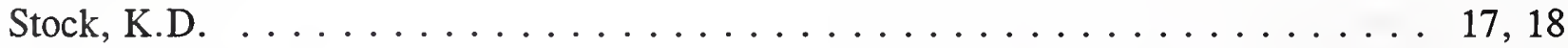

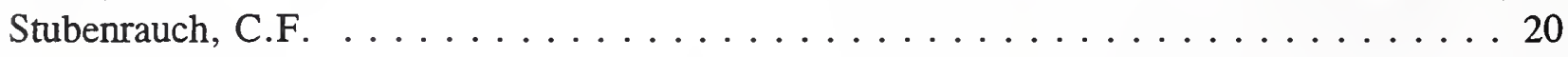

Svalgaard, M. . . . . . . . . . . . . . . . . . . . 59

Swann, W.C. . . . . . . . . . . . . . . . . . . . . . . 59

Synowicki, R.A. . . . . . . . . . . . . . . . . . . . . . 69

Takada, K. . . . . . . . . . . . . . . . . . 66

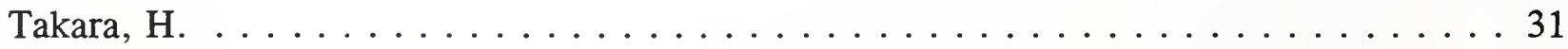

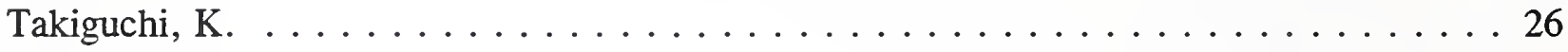

Tang, D. . . . . . . . . . . . . . . 52, 54-57, 67

Tewari, R. . . . . . . . . . . . . . . . . . . . 32

Thomas, B.N. . . . . . . . . . . . . . . . . 49, 52

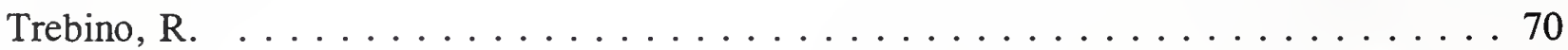

Tseng, W.F. . . . . . . . . . . . . . . . . . 73

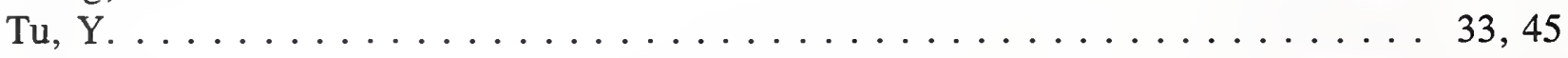

Tuttle, G. . . . . . . . . . . . . . . . . . . 71

Ulu, G. . . . . . . . . . . . . . . . . . . . . 69-71

Ünlü, M.S. . . . . . . . . . . . . . . . . . . . . . . 69-71

Van Engen, A.G. . . . . . . . . . . . . . . . . . . . 9

Vander Rhodes, G.H. . . . . . . . . . . . . . . . . . . . 69-71

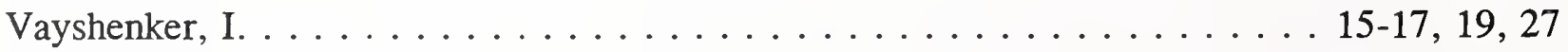

Veasey, D.L. . . . . . . . . . . . . . . . . . . . 63-68

Vecchia, D.F. . . . . . . . . . . . . . . . 26, 29, 30, 32



Veigl, I. . . . . . . . . . . . . . . . . . . . . 65 


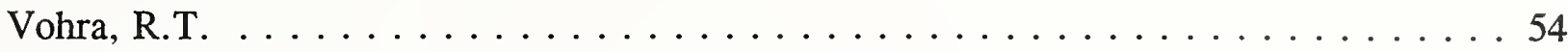

Wadas, A. . . . . . . . . . . . . . . . . . . . . . 64

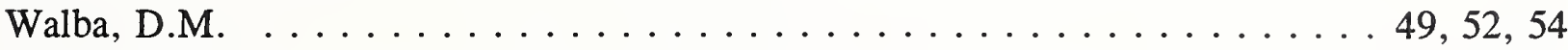

Wand, M.D. . . . . . . . . . . . . . . . . . . . . . . 54

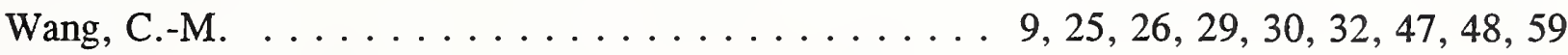

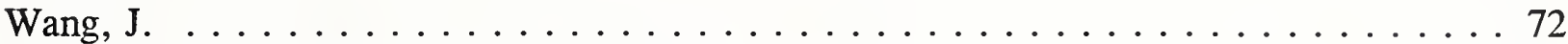



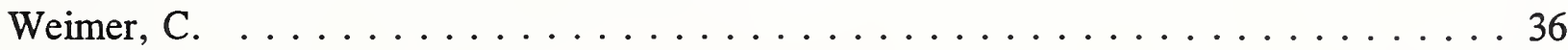



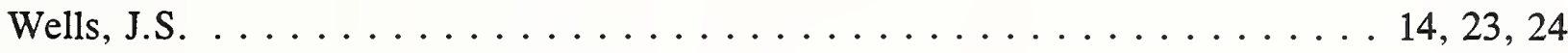

Weppner, M.B. . . . . . . . . . . . . . . . . . . 35, 77

Werner, P.E. . . . . . . . . . . . . . . . . . . . . 13

West, E.D. . . . . . . . . . . . . . . . . . . . . . 22-24

West, J.W. . . . . . . . . . . . . . . . . . . . . 46

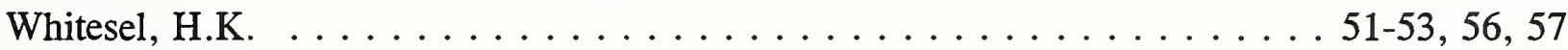

Whittenberg, C.D. . . . . . . . . . . . . . . . . 34, 35

Wieman, C.E. . . . . . . . . . . . . . . . . . . . . 59-61

Wiesendanger, R. . . . . . . . . . . . . . . . . . . . 64

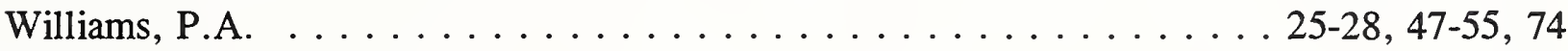

Wineland, D.J. . . . . . . . . . . . . . . . . . . . 61

Wittmann, R.C. . . . . . . . . . . . . . . . . . . 25, 30

Wolfe, R. . . . . . . . . . . . . . . . . . . . . . . 52-54

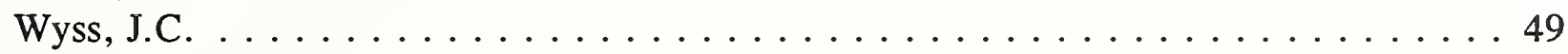

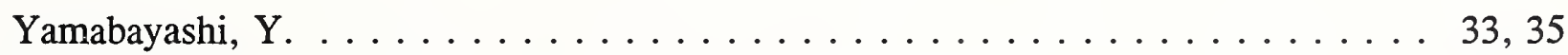

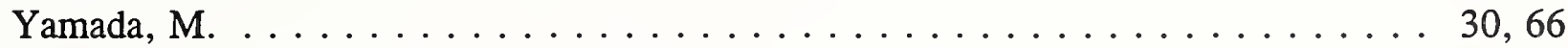

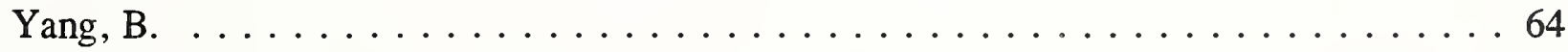

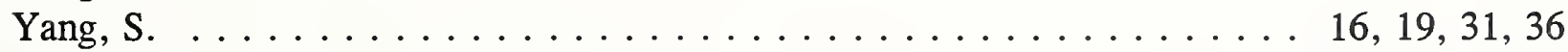

Young, M. . . . . . . . . 20. 25-35, 37-42, 74, 77-79

Zander, M. . . . . . . . . . . . . . . . . . . . 16

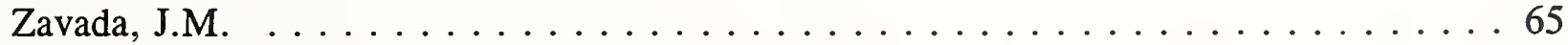

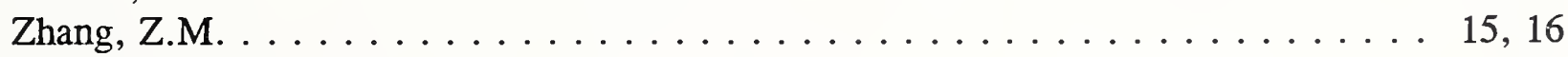

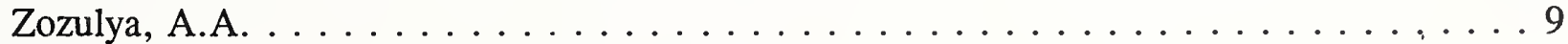




